\title{
Stellar rotation periods determined from simultaneously measured Ca II H\&K and Ca II IRT lines
}

\author{
M. Mittag ${ }^{1}$, A. Hempelmann ${ }^{1}$, J. H. M. M. Schmitt ${ }^{1}$, B. Fuhrmeister ${ }^{1}$, J. N. González-Pérez ${ }^{1}$, and K.-P. Schröder ${ }^{2}$ \\ 1 Hamburger Sternwarte, Universität Hamburg, Gojenbergsweg 112, 21029 Hamburg, Germany \\ e-mail: mmittag@hs .uni-hamburg.de \\ 2 Department of Astronomy, University of Guanajuato, 36000 Guanajuato, Mexico
}

Received 15 December 2016 / Accepted 10 August 2017

\begin{abstract}
Aims. Previous studies have shown that, for late-type stars, activity indicators derived from the Ca II infrared-triplet (IRT) lines are correlated with the indicators derived from the Ca II H\&K lines. Therefore, the Ca II IRT lines are in principle usable for activity studies, but they may be less sensitive when measuring the rotation period. Our goal is to determine whether the Ca II IRT lines are sufficiently sensitive to measure rotation periods and how any Ca II IRT derived rotation periods compare with periods derived from the "classical" Mount Wilson $S$-index.

Methods. To analyse the Ca II IRT lines' sensitivity and to measure rotation periods, we define an activity index for each of the Ca II IRT lines similar to the Mount Wilson $S$-index and perform a period analysis for the lines separately and jointly.

Results. For eleven late-type stars we can measure the rotation periods using the Ca II IRT indices similar to those found in the Mount Wilson $S$-index time series and find that a period derived from all four indices gives the most probable rotation period; we find good agreement for stars with already existing literature values. In a few cases the computed periodograms show a complicated structure with multiple peaks, meaning that formally different periods are derived in different indices. We show that in one case, this is due to data sampling effects and argue that denser cadence sampling is necessary to provide credible evidence for differential rotation. However, our TIGRE data for HD 101501 shows good evidence for the presence of differential rotation.
\end{abstract}

Key words. stars: activity - stars: chromospheres - stars: late-type - stars: rotation

\section{Introduction}

In addition to mass, the rotation period of a late-type main sequence star is among the most important stellar parameters. Due to the rotation-age-activity connection one can estimate the star's chromospheric activity level (e.g. Noyes et al. 1984) and its age (Barnes 2007), given its rotation period. Furthermore, the measurement of the rotation period at different epochs can - at least in principle - provide information on the differential rotation of the underlying star (Donahue et al. 1996).

The rotation period of a star can be measured via photometry or by using spectral lines that are sufficiently sensitive to chromospheric activity. Both methods rely on the presence of spots or plage regions located on and thus rotating with the star, imprinting a rotational modulation on the photospheric continuum or chromospheric emission line flux. It is clear that the method only works with an inhomogeneous distribution of spotted and plage regions over the star. However, recent results from the Kepler mission show the success of the photometric method through the measurement of the rotation periods of many thousands of stars (e.g. Reinhold et al. 2013; McQuillan et al. 2014); we highlight that all the stars measured by Kepler are quite faint and that no such measurements for brighter stars are available.

For the spectroscopic method, the Ca II H\&K lines are normally used, since these lines are the most sensitive ones to chromospheric activity of all spectral lines in the optical waveband, and many examples of Ca II determined rotation periods can be found in the literature (e.g. Noyes et al. 1984; Donahue et al. 1996; Hempelmann et al. 2016). For the very coolest stars, however, the Ca II H\&K lines are harder and harder to observe (at least with smaller telescopes), because these stars become quite faint at the wavelengths of the Ca II $\mathrm{H} \& \mathrm{~K}$ lines.

A possible alternative to the Ca II H\&K lines is the use of the Ca II infrared triplet (with lines located at 8498, 8542, and $8662 \AA$, thereafter Ca II IRT). In earlier studies the general correlation between the Ca II H\&K and the Ca II IRT lines was shown (see, e.g. Martínez-Arnáiz et al. 2011) and previously obtained results show that the Ca II IRT lines are generally usable to also study chromospheric activity. Whether or not it is possible to measure rotation periods using the Ca II IRT lines and, if so, how the periods derived from the Ca II IRT lines compare to the periods derived from the $\mathrm{Ca}$ II $\mathrm{H} \& \mathrm{~K}$ lines, remain open questions. Since the chromospheric emission measured in the Ca II IRT and $\mathrm{Ca}$ II H\&K lines originates from different chromospheric layers (Montes et al. 2000), periods derived from these lines need not necessarily coincide.

Given that these lines, used as chromospheric proxies, may vary in flux on short time scales, it is clear that in any meaningful comparison study of the Ca II H\&K and Ca II IRT lines one must use strictly simultaneously obtained observations. In this paper we therefore use a sample of such strictly simultaneously carried out monitoring observations. We first present our definition of new Ca II IRT activity indices, which we use to measure the rotation period. Furthermore, we present a method to create a periodogram from a number of different, but simultaneously measured activity indices. The results of the rotation measurements of the Ca II IRT lines are then compared with the results 
Table 1. List of the observed objects.

\begin{tabular}{lcccc}
\hline \hline Object & Type & No. obs. & $V[\mathrm{mag}]$ & $B-V[\mathrm{mag}]$ \\
\hline HD 35296 & BY Dra & 57 & 5.00 & 0.544 \\
HD 37394 & BY Dra & 43 & 6.21 & 0.840 \\
HD 39587 & RS CVn & 41 & 4.39 & 0.594 \\
HD 43162 & BY Dra & 28 & 6.37 & 0.713 \\
HD 72905 & BY Dra & 49 & 5.63 & 0.618 \\
HD 75732 & HPM star & 34 & 5.96 & 0.869 \\
HD 97334 & BY Dra & 28 & 6.41 & 0.600 \\
HD 101501 & Var. star & 34 & 5.31 & 0.723 \\
HD 111395 & BY Dra & 35 & 6.29 & 0.703 \\
HD 115043 & HPM star & 41 & 6.82 & 0.603 \\
HD 118972 & PMS star & 27 & 6.92 & 0.855 \\
HD 131977 & BY Dra & 30 & 5.72 & 1.024 \\
\hline
\end{tabular}

Notes. List of the stars identified by HD number, their type designation as given in the SIMBAD catalogue, the total number of observations, visual magnitude, and the colour index $B-V$ taken from HIPPARCos catalogue ESA (1997). The abbreviations HPM and PMS in the second column stand for "high proper motion" and "pre-main sequence star", respectively. For HD 39587, only 40 data points for the Ca II IRT line at $8542 \AA$ were used for the rotation measurement.

obtained using the Mount Wilson S-index (Vaughan et al. 1978; Baliunas et al. 1995) only; a mean rotation period is estimated and compared with literature values. Finally we discuss to what extent there is evidence of differential rotation in our data and present our conclusions.

\section{Observation, data reduction, and synthetic spectra}

For this investigation we chose twelve stars from our ongoing activity study of stars with spectral types $\mathrm{F}$ to $\mathrm{K}$ with the TIGRE telescope; here we use only data from the first monitoring program from August 2013 to July 2014, selecting those stars with the highest significance in their periodograms derived from their Ca II H\&K indices alone (cf., Hempelmann et al. 2016). In Table 1, the investigated stars are listed with the number of observations, their visual magnitude and their colour index $B-V$ (taken from HIPPARCos catalogue ESA 1997). The stars were observed with the TIGRE telescope, located at the La Luz Observatory (located in central Mexico). TIGRE is a $1.2 \mathrm{~m}$ fully robotic telescope with the fibre-fed Échelle spectrograph HEROS (Heidelberg Extended Range Optical Spectrograph). This spectrograph is equipped with two spectral channels (blue $\&$ red), a spectral resolution of $R \approx 20000$, and a spectral range from $\approx 3800 \AA$ to $\approx 8800 \AA$ with a small gap of $100 \AA$ centred at $5800 \AA$; a more detailed description of the TIGRE facility has been provided by Schmitt et al. (2014).

The data are reduced with TIGRE/HEROS standard reduction pipeline, which is implemented in IDL and based on the IDL reduction package REDUCE by Piskunov \& Valenti (2002). The TIGRE/HEROS standard reduction pipeline is designed as a fully automatic pipeline including wavelength calibration. It contains all necessary reduction steps to reduce Échelle spectra (bias subtraction, order definition, wavelength calibration, spectra extraction, flat fielding); additional information can be found in Hempelmann et al. (2016) and Mittag et al. (2016).

One of the main scientific goals of TIGRE is the longterm monitoring of cool stars. Therefore, an additional part of the TIGRE/HEROS reduction pipeline is the estimation of the instrumental $S$-index (thereafter, $S_{\text {TIGRE }}$ ). This instrumental $S$-index can be converted into the "classical" $S_{\text {MWO-index with }}$ the following linear conversion relation derived by Mittag et al. (2016):

$S_{\mathrm{MWO}}=0.036+20.02 \times S_{\mathrm{TIGRE}}$.

During the course of our analysis we also make use of synthetic spectra computed with the radiation transfer code PHOENIX version 16 (Hauschildt et al. 1999). The PHOENIX code provides atmosphere calculations for novae and supernovae as well as for stars and brown dwarfs. Assuming local thermal equilibrium (LTE), Husser et al. (2013) compute the most recent stellar atmosphere library based on the PHOENIX code. The stellar model spectra used in this work were calculated for the mean stellar parameters listed in Mittag et al. (2013, Table 2); they are based on the models computed by Husser et al. (2013), but the elements H, He, Ca I-III, Na I-II, and Mg I-III are treated in nonLTE, which results in improved descriptions of these lines.

\section{Ca II IRT based activity indices}

\subsection{Index definitions}

To characterise the activity observed in the CaII IRT lines, the subtraction method (e.g. Martínez-Arnáiz et al. 2011) could be used, where the reference spectrum of an inactive star is subtracted from the spectrum of the star of interest. The reference spectrum must come from a star as similar in stellar parameters, such as $B-V, \log g$, and metallicity, as is possible to ensure minimal differences in the photospheric spectra. Naturally, finding such a suitable reference spectrum may pose a problem.

A simpler and more practical method is the definition of an activity index in analogy to the "classical" Mount Wilson $S$ index by determining the ratio between the counts in the line core of each of the Ca II IRT lines and the counts in a reference spectral range. The great advantage of this purely empirical approach is its simplicity with an index directly obtained from the data without any reference to a synthetic or comparison inactive spectrum.

We then define our $S_{\text {IRT-indices for the Ca II IRT lines using }}$ the following equations:

$$
\begin{aligned}
S_{\text {IRT_8498 }} & =\frac{N_{8498}}{N_{\text {CONT_1A }}+N_{\text {CONT_1B }}}, \\
S_{\text {IRT_8542 }} & =\frac{N_{8542}}{N_{\text {CONT_2A }}+N_{\text {CONT_2B }}},
\end{aligned}
$$

and

$S_{\text {IRT_8662 }}=\frac{N_{8662}}{N_{\text {CONT_3A }}+N_{\text {CONT_3B }}}$,

where $N$ is the number of counts in the bandpasses explained below and illustrated in Fig. 1. For our $S_{\text {IRT-indices we always use }}$ an $1 \AA$ bandpass centred in the line core to estimate the counts in the Ca II IRT lines. In Fig. 1, the three Ca II IRT lines with the bandpasses of the respective $S_{\mathrm{IRT}}$-index are shown. Some care in the definition of the locations of the reference bands is required since in the spectral range of the Ca II IRT some telluric lines (mostly $\mathrm{H}_{2} \mathrm{O}$ lines) are located. The line intensity of the telluric lines is time-variable, therefore this must be considered in the definition of the used line continua, and we have selected spectral ranges for the line continua without any or very low telluric line contamination. Since the considered spectral range 
M. Mittag et al.: Comparison measurement of rotation periods with Ca II H\&K and Ca II IRT
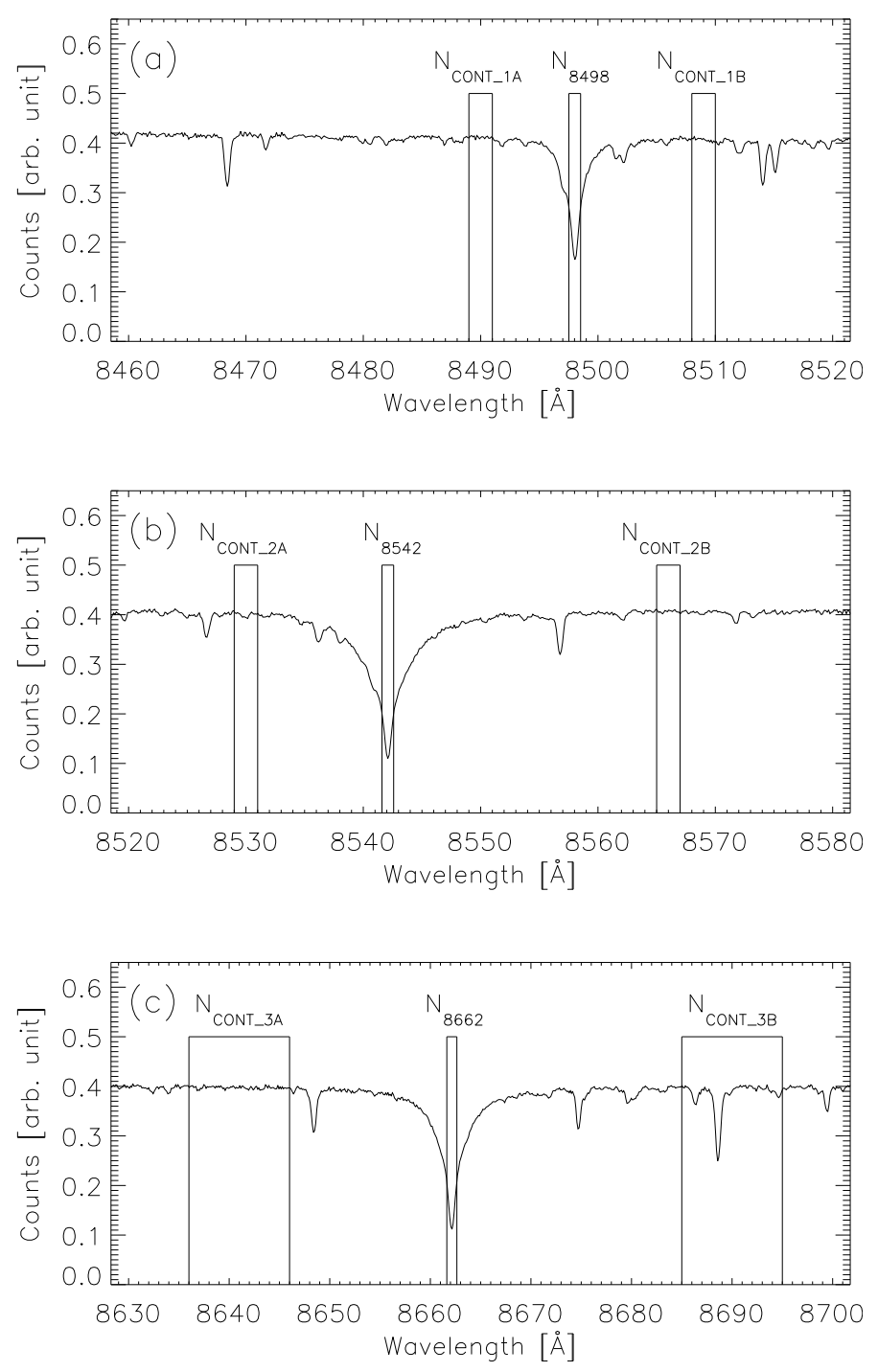

Fig. 1. A TIGRE spectrum of the Sun (moon light) of the Ca II IRT lines with the bandpasses chosen for the definition of the $S_{\text {IRT-indices: the top }}$ panel shows the spectral regions used for the $S_{\text {IRT_8498-index, the middle }}$

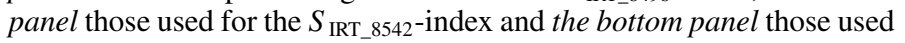
for the $S_{\text {IRT_8662-index. }}$

contains a large number of stronger telluric lines, this resulted in only $2 \AA$ wide bandpasses used for the reference continua for the Ca II IRT lines at 8498 and $8542 \AA$; the defined continua for the Ca II IRT line at $8498 \AA$ are centred at 8490 and $8509 \AA$ and for the Ca II IRT line at 8542,8530 , and $8566 \AA$. For the Ca II IRT line at $8662 \AA$, the line continua are defined as a $10 \AA$ wide bandpass centred at 8641 and $8690 \AA$, respectively. The three lines in the bandpass centred at $8690 \AA$ are stellar lines of the elements $\mathrm{Si}$ I and Fe I. We note that the continuum reference bands are not symmetric with respect to the respective lines.

\subsection{Index renormalisation}

Because of the asymmetric placement of the quasi-continuum reference bands we must use the "true" spectral trend and slope for the considered spectral range $(8450-8720 \AA)$ to obtain $S_{\text {IRT }}$ indices consistent with the respective flux ratios. We adapt the method described by Hall \& Lockwood (1995) to renormalise the CaII $\mathrm{H} \& \mathrm{~K}$ region for measuring the instrumental Mount

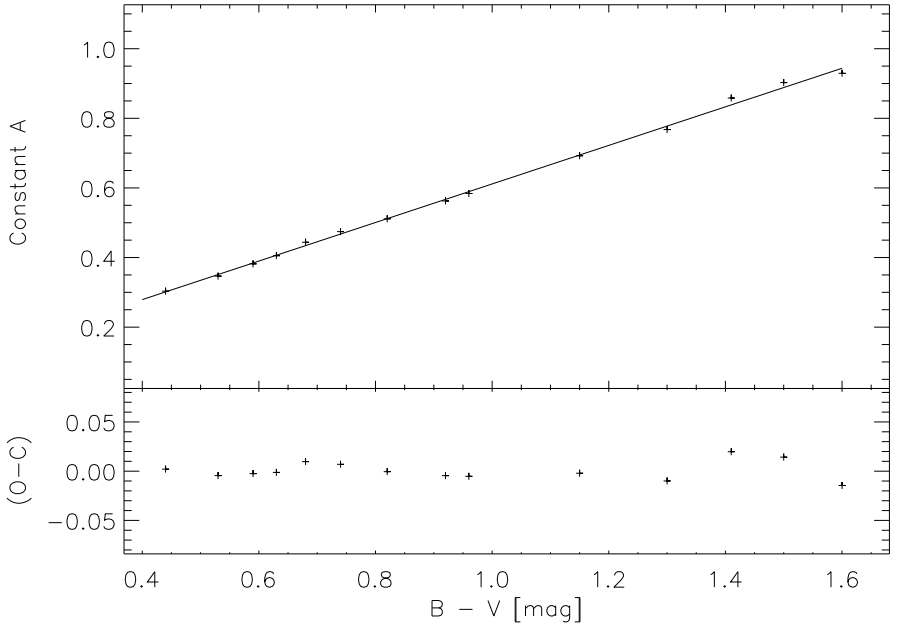

Fig. 2. Upper panel: best fit coefficients of the constant A of the linear fit vs. $B-V$. The solid line represents the determined relation. Lower panel: the residuals.

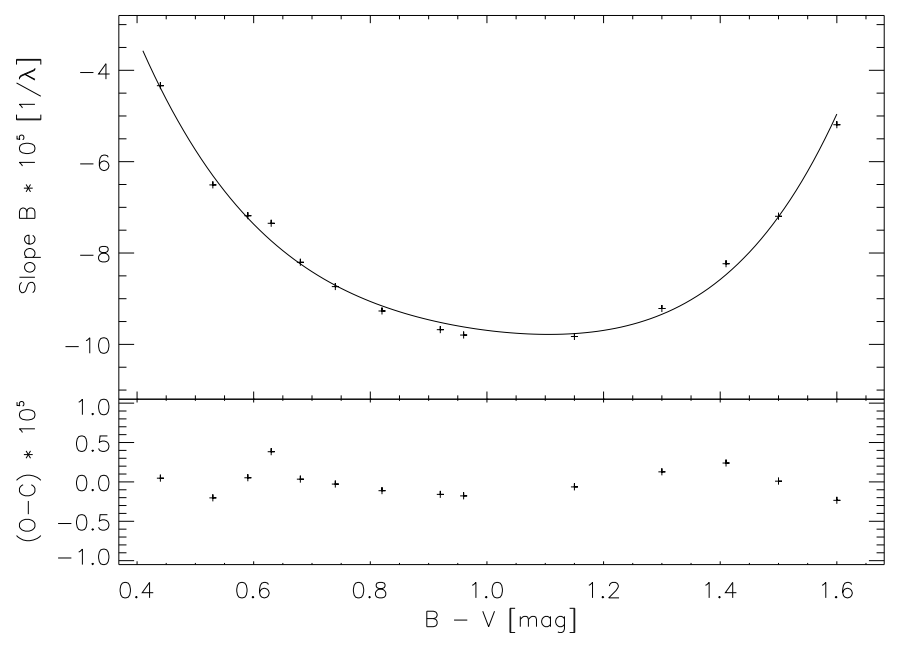

Fig. 3. Upper panel: best fit coefficients of the slope B of the linear fit multiplied with $10^{5}$ vs. $B-V$. The solid line represents the determined relation. Lower panel: the residuals.

Wilson $S$-value for the spectral range of the Ca II IRT. To estimate the spectral slope in the Ca II IRT region, we use synthetic PHOENIX spectra (see Sect. 2), calculated for main sequence stars in the $B-V$ range from 0.44 to 1.6 , as follows: First, we normalise the computed PHOENIX spectrum by its maximal flux value, then we select seven supporting points (at 8456, 8484, $8523,8570,8630,8697$, and $8715 \AA$ ) for the continuum $(x, y)$ and fit a linear function with the approach $y=A+B x$, where $y$ is the normalised flux and $x$ the wavelength. The resulting fit coefficients - the constant $A$ and the slope $B-$ are then functions of the colour $B-V$ and are plotted against $B-V$ in Figs. 2 and 3 .

Finally, we empirically determine a relation describing the dependence of the constant $A$ and the slope $B$ on the $B-V$-colour to calculate a correction function $\operatorname{corr}(\lambda, B-V)$ to renormalise the whole Ca II IRT range. For the correction function in the spectral range $8450-8720 \AA$, we obtain the following relations:

$\operatorname{corr}(\lambda, B-V)=A(B-V)+B(B-V) x$, 
Table 2. The mean $S$-indices of the Ca II H\&K and Ca II IRT lines and the standard deviation $(\sigma)$.

\begin{tabular}{lcccccccc}
\hline \hline Object & $\overline{S_{\text {MWO }}}$ & $\sigma_{S_{\text {MWO }}}$ & $\overline{S_{\text {IRT_8498 }}}$ & $\sigma_{S_{\text {IRT \&4898 }}}$ & $\overline{S_{\text {IRT_8542 }}}$ & $\sigma_{S_{\text {IRT } 8542}}$ & $\overline{S_{\text {IRT_8662 }}}$ & $\sigma_{S_{\text {IRT _8662 }}}$ \\
\hline HD 35296 & 0.319 & 0.006 & 0.1566 & 0.0017 & 0.1258 & 0.0017 & 0.02561 & 0.00034 \\
HD 37394 & 0.457 & 0.015 & 0.1445 & 0.0015 & 0.1151 & 0.0018 & 0.02295 & 0.00036 \\
HD 39587 & 0.322 & 0.011 & 0.1513 & 0.0015 & 0.1200 & 0.0044 & 0.02433 & 0.00041 \\
HD 43162 & 0.416 & 0.021 & 0.1558 & 0.0028 & 0.1274 & 0.0036 & 0.02521 & 0.00068 \\
HD 72905 & 0.397 & 0.009 & 0.1620 & 0.0016 & 0.1332 & 0.0025 & 0.02666 & 0.00036 \\
HD 75732 & 0.178 & 0.008 & 0.1140 & 0.0006 & 0.0801 & 0.0006 & 0.01650 & 0.00011 \\
HD 97334 & 0.354 & 0.012 & 0.1518 & 0.0017 & 0.1228 & 0.0020 & 0.02459 & 0.00034 \\
HD 101501 & 0.333 & 0.013 & 0.1440 & 0.0017 & 0.1116 & 0.0020 & 0.02261 & 0.00039 \\
HD 111395 & 0.260 & 0.016 & 0.1331 & 0.0016 & 0.0987 & 0.0019 & 0.02041 & 0.00037 \\
HD 115043 & 0.313 & 0.011 & 0.1484 & 0.0020 & 0.1165 & 0.0025 & 0.02362 & 0.00034 \\
HD 118972 & 0.570 & 0.018 & 0.1576 & 0.0016 & 0.1292 & 0.0025 & 0.02587 & 0.00037 \\
HD 131977 & 0.462 & 0.026 & 0.1236 & 0.0014 & 0.0914 & 0.0017 & 0.01881 & 0.00030 \\
\hline
\end{tabular}

where $x=\lambda-8450 \AA$ and the relations for the constant and slope are:

$$
\begin{aligned}
A(B-V)= & 0.2791+0.5539 z \\
B(B-V)= & -3.28 \times 10^{-5}-2.98 \times 10^{-4} z \\
& +5.71 \times 10^{-4} z^{2}-5.63 \times 10^{-4} z^{3} \\
& +2.37 \times 10^{-4} z^{4}
\end{aligned}
$$

where $z=(B-V)-0.4$.

We now briefly describe our estimation of the $S_{\text {IRT }}$-indices using the Ca II IRT line at $8498 \AA$ as an example. First, the wavelength is corrected with the barycentric velocity shift. Next, the Ca II IRT line region is continuum-normalised. Then we measure the radial velocity by a cross-correlation with a synthetic PHOENIX spectrum, taking into account the rotational linebroadening by $v \sin (i)$ and the spectral resolution of the spectrograph $(R \approx 20000)$. After the radial velocity correction, the "true" tilt of the spectrum is calculated with the correction function Eq. (5) and multiplied by the continuum-normalised spectrum. From this spectrum we calculate the $S_{\text {IRT_8498-index using }}$ Eq. (2). The counts in the bandpasses are integrated and the ratio is calculated; to estimate the errors of the individual bandpasses, we perform a Monte-Carlo simulation for each bandpass, taking into account the signal-to-noise ratio in each single pixel. The standard deviation of the integral variations is used as the error of the corresponding bandpass. Finally the error of the $S_{\text {IRT_8498- }}$ index is estimated by error propagation of the errors of the single bandpasses.

\subsection{Ca II based indices and correlations}

For the sample stars considered in this paper, the mean $S_{\text {MWO }}$ and $S_{\text {IRT }}$ indices and the corresponding standard deviations are

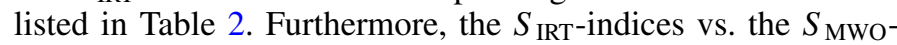
indices are plotted for each sample star in Figs. A.1-A.12. An example is shown in Fig. 4 for the $S_{\text {IRT_8498 Vs. }} S_{\text {MwO }}$ indices measured by TIGRE for the star HD 131977. Obviously, the two indices appear highly correlated, and in order to quantify the correlation between the $S_{\text {MwO }}$ and $S_{\text {IRT }}$-indices we calculate Spearsman's $\rho$ correlation coefficients for all of our sample stars and list the results in Table 3. As is clear from Table 3, for most stars, the $S_{\mathrm{IRT}^{-}}$and $S_{\mathrm{MWO}}$-indices are clearly correlated, and only for a few stars, do we find little or no correlation between the various indices. In Fig. 5 we plot the derived values of $\rho$ between the three Ca II IRT lines and Ca II H\&K lines as a function of the measured dispersion in the $S_{\mathrm{MWO}}$-indices and find
Table 3. The correlation coefficient (Spearsman's $\rho$ ) between $S_{\text {MwO }}$ and $S_{\text {IRT-indices time series. }}$

\begin{tabular}{lccc}
\hline \hline Object & $S_{\text {IRT_8498 }}$ & $S_{\text {IRT_8542 }}$ & $S_{\text {IRT_8662 }}$ \\
\hline HD 35296 & 0.253 & 0.387 & 0.381 \\
HD 37394 & 0.578 & 0.710 & 0.400 \\
HD 39587 & 0.641 & 0.600 & 0.551 \\
HD 43162 & 0.890 & 0.931 & 0.943 \\
HD 72905 & 0.583 & 0.572 & 0.720 \\
HD 75732 & 0.191 & 0.276 & 0.140 \\
HD 97334 & 0.496 & 0.682 & 0.656 \\
HD 101501 & 0.626 & 0.436 & 0.685 \\
HD 111395 & 0.704 & 0.595 & 0.592 \\
HD 115043 & 0.581 & 0.684 & 0.812 \\
HD 118972 & 0.731 & 0.617 & 0.812 \\
HD 131977 & 0.924 & 0.934 & 0.896 \\
\hline
\end{tabular}

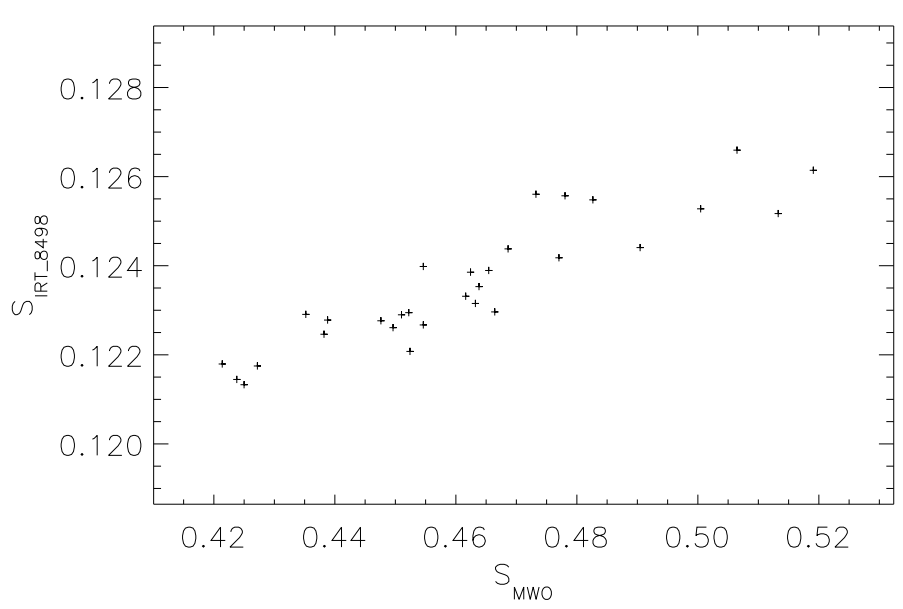

Fig. 4. Correlation between $S_{\text {IRT } 8498}$ and $S_{\text {MWO }}$ for HD 131977 with a Spearman's $\rho$ of 0.924 .

that stars with a larger spread in the $S_{\mathrm{MwO}}$-index tend to show a larger correlation coefficient. The significance of the correlations between $\sigma\left(S_{\mathrm{MWO}}\right)$ and $\rho\left(S_{\mathrm{MWO}^{-}} S_{\text {IRT_8498 }}\right)$, and $\rho\left(S_{\mathrm{MWO}^{-}}\right.$ $\left.S_{\text {IRT_8542 }}\right)$ and $\rho\left(S_{\text {MWO }}-S_{\text {IRT_8662 }}\right)$ are $99.9 \%, 99.7 \%$, and $99.3 \%$. There are two possible explanations for the trends. First, the Ca II IRT lines may have a lower sensitivity to stellar activity. Second, noise may be a problem, hiding, especially for the lowest dispersion values, any underlying correlation. 
M. Mittag et al.: Comparison measurement of rotation periods with Ca II H\&K and Ca II IRT

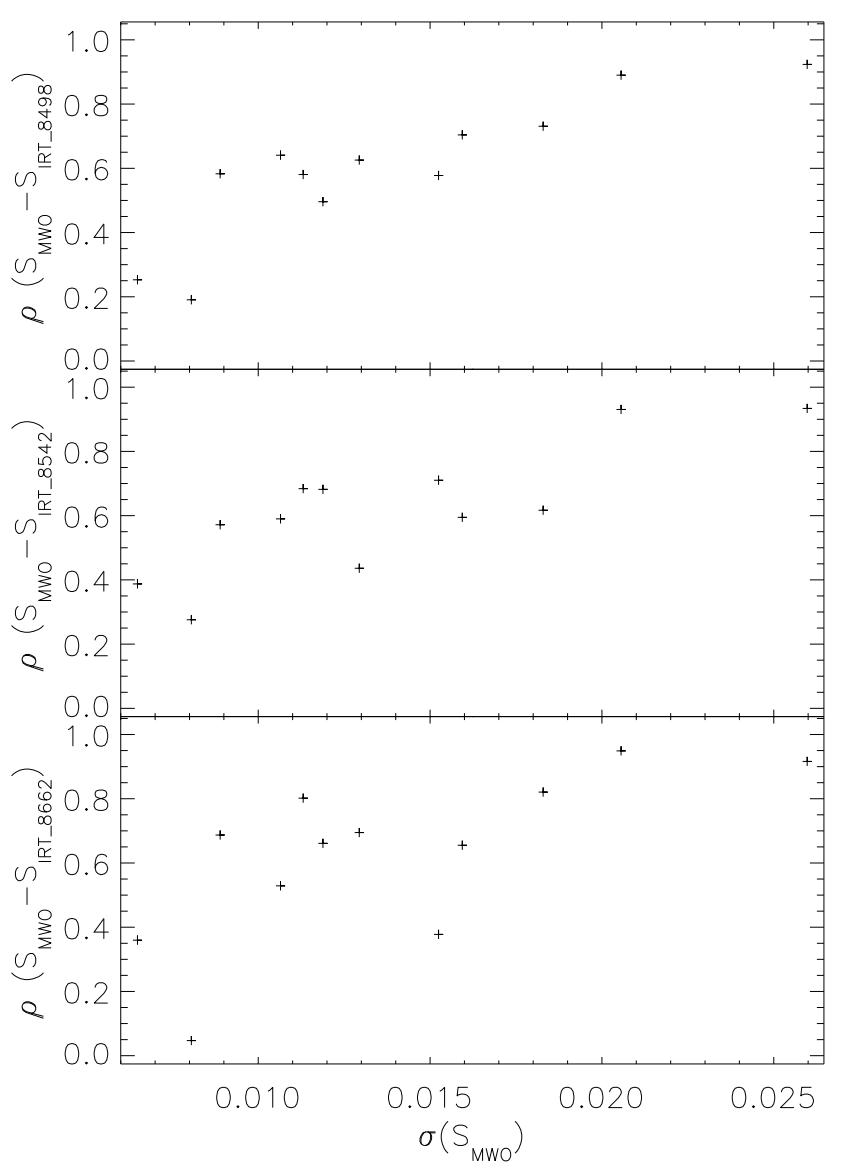

Fig. 5. Spearman's $\rho$ correlation coefficients of the correlation between $S_{\mathrm{MWO}}$ and $S_{\mathrm{IRT}}$-indices vs. the standard deviation $(\sigma)$ of the $S_{\mathrm{MWO}}$ time series.

\section{Period and significance estimation}

\subsection{Detrending}

Before analysing our time series for periodic variations, we have to determine and remove possible trends in the data; such trends could be caused, for example, by long-term trends in the activity of the sample stars. For our study, the precise nature of these trends is not relevant, however, detrending is important for the period estimation, since an unaccounted trend can attenuate the power derived in the periodogram. The common methods to search for periodicities are the Lomb-Scargle periodogram (Lomb 1976; Scargle 1982; Horne \& Baliunas 1986) or the generalized Lomb-Scargle periodogram (thereafter GLS; Zechmeister \& Kürster 2009), which assume a purely sinusoidal wave form, and the GLS by Zechmeister \& Kürster (2009), which additionally takes into account a constant offset, but no systematic trend.

We perform detrending by using polynomials of varying order. To decide which order of polynomial is appropriate, we detrend the time series with polynomials of different order, analyse the periodic variations via GLS, and estimate the period with the largest peak height. The thus determined period is then removed from the data via a sinusoidal wave fit. To estimate the significance of the trend, the $F$-test is used to estimate the significance of the reduction of the scatter in the residuals. The criterion for finding the most suitable order of the polynomial fit is the height of the largest peak (maximal power $(p(\omega))$ ) and hence the significance of the corresponding period. Furthermore, the results of the $F$-test are used to check the significance of the used

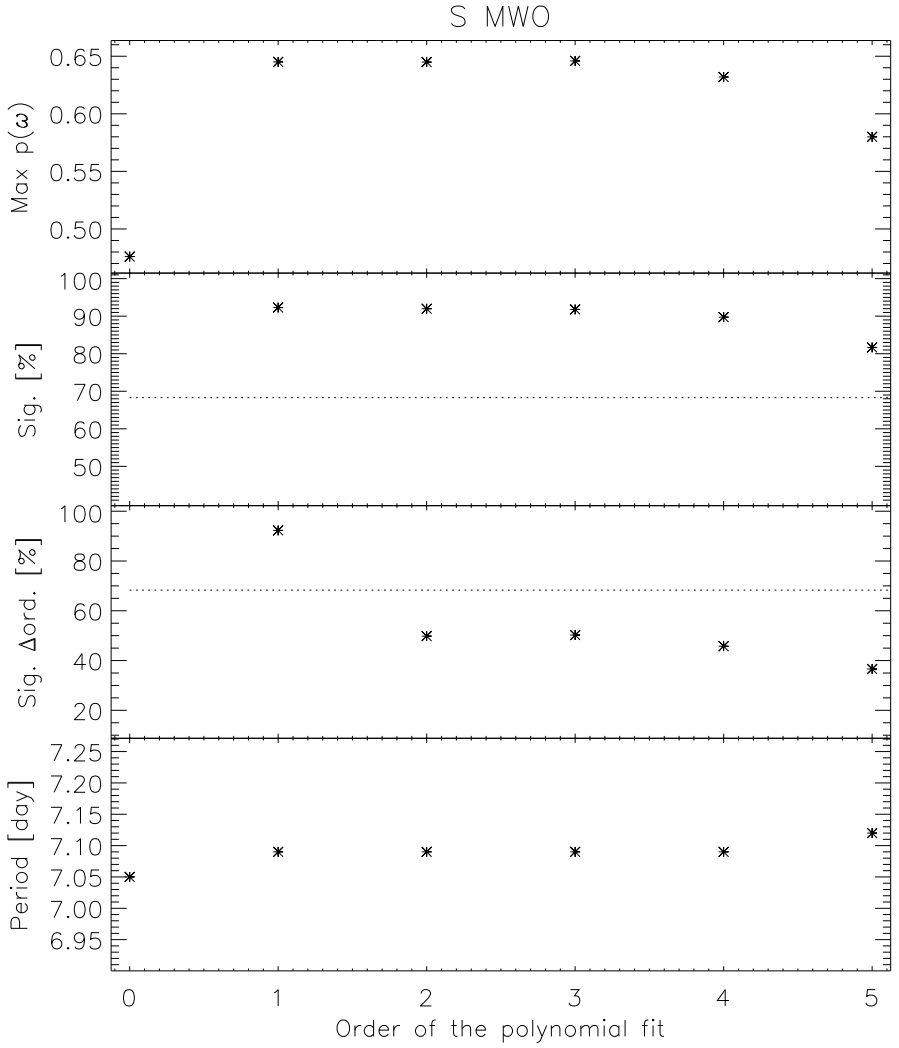

Fig. 6. Example for the estimation of the polynomial order for the detrending of the $S_{\text {Mwo }}$ time series of HD 43162: the upper panel shows the maximal power $p(\omega)$, the panel below the results of the $F$-test, where the significance of the scatter reduction is compared to the residuals of the non-detrended time series, the third panel shows the significance of the scatter reduction compared to detrending with the polynomial fit of order $\mathrm{N}-1$ and in the lowest panel we plot the estimated periods; the dotted lines show the formal $1 \sigma$ limit.

polynomial fit and so to find the optimal order of the polynomial fit. Here, the significance limit of the scatter reduction is set to " $1 \sigma$ " because the intrinsic scattering is relatively large, meaning that a higher limit is inappropriate.

As an example of our procedure we consider the case of HD 43162 (see Figs. 6 and A.4). In the upper panel, the maximal peak heights $(\operatorname{Max} p(\omega))$ are shown as a function of the polynomial order and it is apparent that the peak height increases significantly with a detrending polynomial of first order. The following panel shows the significance of the scatter reduction compared to the residuals of the non-detrended time series. In the third panel of Fig. 6, the significance of the scattering reduction compared to the detrending with the polynomial fit of order $N-1$ are shown to check whether the significance changes exceed the $1 \sigma$ level. It is obvious that a fit with a polynomial of order larger than 1 is not warranted. In the lower panel, the periods vs. polynomial order are depicted to show the dependence of the derived period on the order of the polynomial.

Finally, we point out that the detrending of the investigated time series is a somewhat controversial issue, because the details of the applied detrending procedures can influence the resulting periodograms. To quantify the influence of the detrending polynomial on the period, we calculate the ratio between the standard deviation and mean of the periods for the different polynomial fits (see the listing in Table 4). The periods do not show any significant dependencies on the used polynomial orders and are stable to within a few percent, except for the case of HD 75732, 
Table 4. Ratio between the standard deviations of the periods and the mean period obtained from the different polynomial orders for the detrending.

\begin{tabular}{lcccc}
\hline \hline Object & $\sigma / \bar{P}$ & $\sigma / \bar{P}$ & $\sigma / \bar{P}$ & $\sigma / \bar{P}$ \\
& $S_{\text {MWO }}$ & $S_{\text {IRT_8498 }}$ & $S_{\text {IRT_8542 }}$ & $S_{\text {IRT_8662 }}$ \\
\hline HD 35296 & 0.015 & 0.002 & 0.002 & 0.001 \\
HD 37394 & 0.002 & 0.002 & 0.003 & 0.048 \\
HD 39587 & 0.016 & 0.002 & 0.016 & 0.028 \\
HD 43162 & 0.003 & 0.003 & 0.003 & 0.003 \\
HD 72905 & 0.002 & 0.001 & 0.089 & 0.004 \\
HD 75732 & 0.055 & 0.205 & 0.186 & 0.348 \\
HD 97334 & 0.005 & 0.006 & 0.016 & 0.028 \\
HD 101501 & 0.006 & 0.022 & 0.004 & 0.005 \\
HD 111395 & 0.006 & 0.005 & 0.005 & 0.005 \\
HD 115043 & 0.002 & 0.002 & 0.002 & 0.002 \\
HD 118972 & 0.001 & 0.002 & 0.004 & 0.003 \\
HD 131977 & 0.012 & 0.017 & 0.005 & 0.003 \\
\hline
\end{tabular}

where no significant rotation period was found in the Ca II IRT lines. Furthermore, the periods vs. polynomial orders are shown in the corresponding panels in Figs. A.1-A.12 to visualise the general problem of detrending.

\subsection{Period determination}

For the search for periodic variations in the time series of the Ca II H\&K and Ca II IRT lines, we carry out a Fourier analysis using the GLS method by Zechmeister \& Kürster (2009). Given $M$ data values $y_{j}$ at times $t_{j}$ and uncertainties $\sigma_{j}$, the formalism computes a $\chi^{2}$ value with the approach $y_{j}=A \sin \omega t_{j}+$ $B \cos \omega t_{j}+C$, with constants $A, B$ and $C$ determined from the data, to generate a periodogram as a function of the chosen frequency $\omega$. The periodogram is computed from the following expression (Zechmeister \& Kürster 2009);

$p(\omega)=\frac{\chi_{0}^{2}-\chi^{2}(\omega)}{\chi_{0}^{2}}=1-\frac{\chi^{2}(\omega)}{\chi_{0}^{2}}$,

where $\chi_{0}^{2}$ is a value for the dispersion around the mean and is used for the normalisation of $\chi^{2}(\omega)$; the detailed formulae to compute $p(\omega)$ can be found in Zechmeister \& Kürster (2009).

In our case, we are dealing not only with a single series of data $y_{j}$ at times $t_{j}$, but rather we are dealing with a total of four index time series $y_{i, j}$, where $i=1,4$ and $j=1, M$, each representing one of our Ca II index time series. If one is not interested in the phase of the different time series we can "combine" the periodograms in the following way: Zechmeister \& Kürster (2009) show that the normalised power spectrum $p(\omega)$ (with the cross terms set to zero by suitably shifting the times $t_{j}$ ) can be written as (Eq. (5) in their paper)

$p(\omega)=\frac{Y C^{2}}{Y Y \times C C}+\frac{Y S^{2}}{Y Y \times S S}$,

where the nomenclature is the same as in Zechmeister \& Kürster (2009). The two sums in Eq. (9) extend over all the data points in the given data set at some frequency $\omega$. In our case, several data sets provide information on the power at frequency $\omega$. We therefore consider the mean power (averaged over the $N$ data

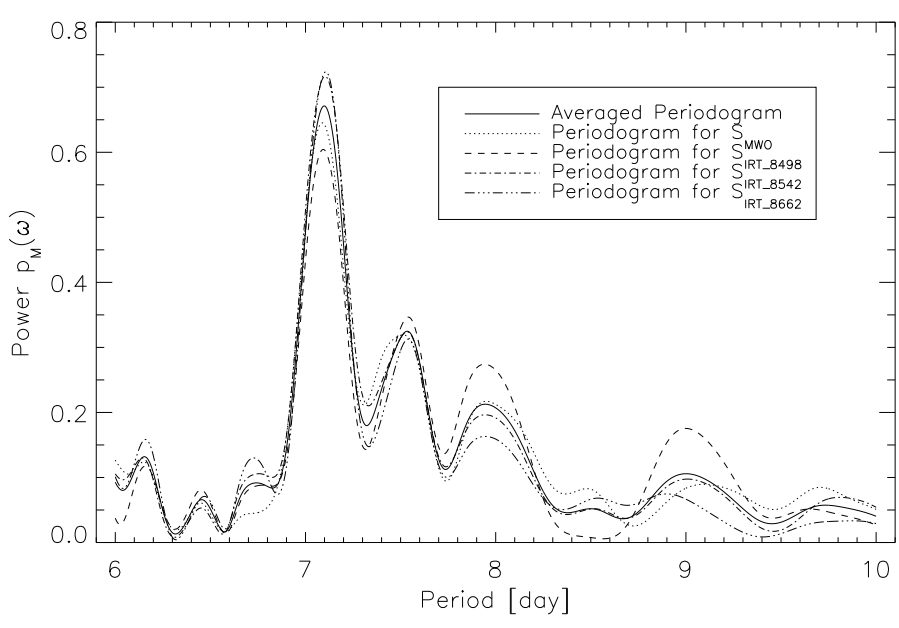

Fig. 7. Averaged periodogram and individual periodograms from the $S_{\text {MWO }}$ and the $S_{\text {IRT }}$ time series for HD 43162; the solid line depicts the averaged periodogram, the dotted line the $S_{\text {MWO }}$, the dashed line the $S_{\text {IRT_8498 }}$, the dashed-dotted line the $S_{\text {IRT_8542 }}$ and the dashed-tripledotted line the $S_{\text {IRT_8662 }}$ periodograms.

sets) through

$p_{\mathrm{M}}(\omega)=\frac{1}{N} \sum_{i=1}^{N} p_{i}(\omega)=\frac{1}{N} \sum_{i=1}^{N} \frac{Y C_{i}^{2}}{Y Y_{i} \times C C_{i}}+\frac{Y S_{i}^{2}}{Y Y_{i} \times S S_{i}}$,

where the quantities subscripted with the index $i$ refer to the $i$ th data set. Since the data sets are independent (the phases do not need to agree) we can use the formulae given in the Appendix of Zechmeister \& Kürster (2009) for each data set and find

$p_{\mathrm{M}}(\omega)=\frac{1}{N} \sum_{i=1}^{N}\left(1-\frac{\chi_{i}^{2}(\omega)}{Y Y_{i} W_{i}}\right)=\frac{1}{N} \sum_{i=1}^{N}\left(1-\frac{\chi_{i}^{2}(\omega)}{\chi_{0, i}^{2}}\right)$,

which is a simple averaged periodogram.

In this fashion we can "average" the periodograms from our four line indices into a single, mean periodogram, an example of which is shown in Fig. 7. Here, the averaged periodogram and the original periodograms of the four time series for HD 43162 are shown.

\subsection{Period errors, significance, and FAP estimation}

To estimate the error on the determined period we use the equation given by Baliunas et al. (1995),

$\Delta P=\frac{3 \sigma_{n} P^{2}}{4 T A \sqrt{N}}$,

where $P$ is the period, $\sigma_{n}$ the standard deviation of the residuals (O-C), $T$ the total length of the observation interval, $A$ the amplitude of the signal, and $N$ the total number of data points.

For the most probable rotation period $\left(P_{\mathrm{M}}\right)$ obtained from the averaged periodogram, the error is estimated in the same fashion. Here, the amplitude and the $\sigma_{n}$ for each time series are estimated with the period found in the averaged periodogram and the values of $\Delta P$ for each time series are estimated. Finally, the $\Delta P$ values of each time series $\left(\Delta P_{i}\right)$ are combined to the error of the most probable rotation period $\left(\Delta P_{\mathrm{M}}\right)$ with the formalism to estimate the error of the weighted mean:

$\Delta P_{\mathrm{M}}=\frac{1}{\sqrt{\sum_{i=0}^{N} \Delta P_{i}^{-2}}} \cdot$ 


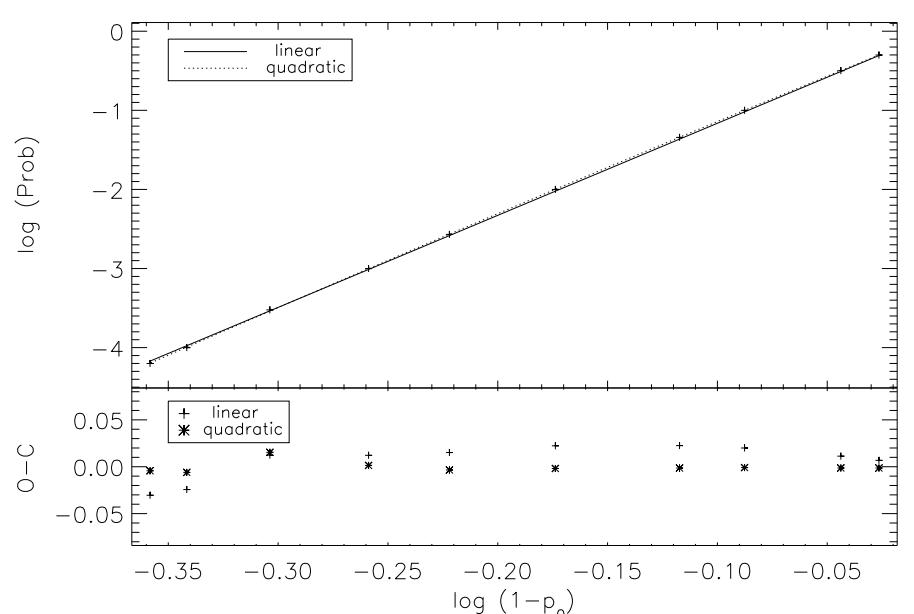

Fig. 8. Significance estimation of the $S_{\text {Mwo }}$ time series for HD 43162: Upper panel: $\operatorname{logarithm}$ of the probability $\left(\operatorname{Prob}\left(p>p_{0}\right)\right)$ vs. logarithm of one minus significance level $\left(1-p_{0}\right)$. The solid line shows the linear fit and the dotted line the quadratic fit. Lower panel: the residuals of the linear fit are depicted as crosses and the quadratic fit as asterisks.

To estimate the probability levels in the periodogram, a bootstrap method is used, permuting the recorded data $10^{5}$ times, while maintaining the times of the measurements. With this method, we cannot estimate the exact probability of a peak in the periodogram, but we can estimate probability limits. Given the probabilities and the corresponding significance levels, we can derive a general relation between the peak height and the probability in order to estimate the peak probability. Therefore, the approach for the estimation of the probabilities is used from Zechmeister \& Kürster (2009):

$\operatorname{Prob}\left(p>p_{0}\right)=\left(1-p_{0}\right)^{\frac{N-3}{2}}$,

where $p_{0}$ is the peak height and $N$ the number of data points. The exponent $\frac{N-3}{2}$ is a normalisation factor for the periodogram to convert the power into a probability. This exponent can be substituted so that we obtain

$\operatorname{Prob}\left(p>p_{0}\right)=\left(1-p_{0}\right)^{Z}$

or

$\log \left(\operatorname{Prob}\left(p>p_{0}\right)\right)=Z \log \left(1-p_{0}\right)$.

The latter equation describes a linear relation between the logarithmic probability and the logarithm of the peak height with the normalisation factor as the slope.

In Fig. 8 we present an example of our procedures and plot the logarithmic probabilities against the logarithm of the peak heights for the bootstrapped GLS periodograms for HD 43162; we find that a linear relation describes the general trend of the relation between the logarithmic probability and logarithm of the peak height. In this particular case we obtain a slope of 11.7 which differs slightly from the calculated value for the exponent of 12.5 (using $N=28$, see Eq. (14)). Furthermore, a visual inspection of the residuals shows a systematic deviation from a linear curve. We therefore add a quadratic correction term to Eq. (16) and write

$\log \left(\operatorname{Prob}\left(p>p_{0}\right)\right)=a \log \left(1-p_{0}\right)+b \log \left(1-p_{0}\right)^{2}$,

which provides a better description of the relation between the logarithmic probability and logarithm of the peak height. The

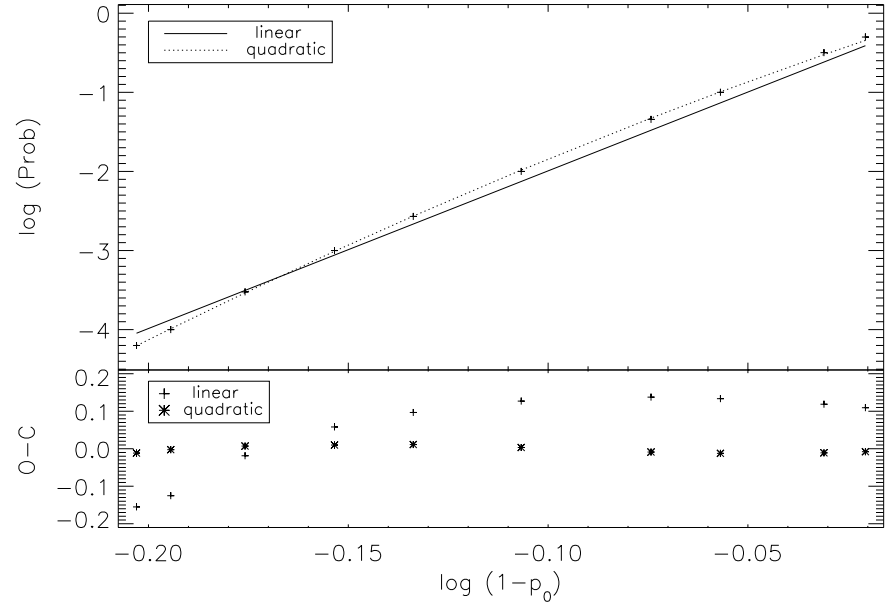

Fig. 9. Significance estimation of the signal in the averaged periodogram for HD 111395: Upper panel: logarithm of the probability $\left(\operatorname{Prob}\left(p>p_{0}\right)\right)$ vs. logarithm of one minus significance level $\left(1-p_{0}\right)$. The solid line shows the linear fit and the dotted line the quadratic fit. Lower panel: the residuals of the linear fit are depicted as crosses and the quadratic fit as asterisks.

standard deviation of the residuals for the linear fit is 0.019 and for the quadratic fit is 0.006 . The $F$-test gives a significance of $\approx 99.5 \%$ for the scatter reduction with the quadratic fit compared to the linear fit. Another example for the quadratic fit is shown in Fig. 9, where we show the significance estimation for the averaged periodogram of the four time series for HD 111395; it is obvious that the quadratic fit describes this relationship better than the linear fit: the standard deviation of the residuals is $\approx 0.11$ for the linear fit and 0.02 for the quadratic fit; and with the $F$-test, we obtain a significance $>99.9 \%$ for the scatter reduction with the quadratic fit compared to the linear fit. Needless to say, in some cases, a linear fit is possible, but for consistency we use the quadratic fit.

In comparison to Eq. (14), the normalisation factor is estimated independently from the number of data points and we obtain a more accurate peak probability.

With Eq. (17), the probability of a peak can be calculated, but to estimate the false alarm probability (thereafter, FAP) of the peak one needs the number of independent frequencies. To estimate these frequencies we use the method described by Zechmeister \& Kürster (2009) and assume the number of independent frequencies $M=\frac{\Delta f}{\delta f}$, where $\Delta f\left(f_{\max }-f_{\min }\right)$ is the frequency range and $\delta f$ the frequency resolution. The frequency resolution is estimated as the full width at half maximum (FWHM) of a peak in the periodogram. The frequency range corresponds to the highest reasonable frequency $\left(f_{\max }\right)$ because the time series are long enough to assume $f_{\max } \gg f_{\min }$ and so that applies $\Delta f=f_{\max }$ (Zechmeister \& Kürster 2009). To estimate the highest reasonable frequency, the time spans between consecutive observations $(\Delta T)$ are calculated. and the most frequent value of $\Delta T$ is multiplied by two to obtain the smallest reasonable period and so the highest reasonable frequency. This definition is based on the definition of the Nyquist frequency for evenly sampled time series.

Finally, following Zechmeister \& Kürster (2009) the FAP is estimated as

FAP $=1-\left[1-\operatorname{Prob}\left(p>p_{0}\right)\right]^{M}$,

where $M$ is the number of independent frequencies and $\operatorname{Prob}\left(p>p_{0}\right)$ is estimated with Eq. (17). 
Table 5. Periods found using the $S_{\text {MWO }}$ and $S_{\text {IRT }}$ time series with the corresponding FAP.

\begin{tabular}{|c|c|c|c|c|c|c|c|c|c|}
\hline \multirow[b]{2}{*}{ Object } & \multirow[b]{2}{*}{ Figure } & \multicolumn{2}{|c|}{$S_{\text {MWO }}$} & \multicolumn{2}{|c|}{$S_{\text {IRT_8498 }}$} & \multicolumn{2}{|c|}{$S_{\text {IRT_8542 }}$} & \multicolumn{2}{|c|}{$S_{\text {IRT_8662 }}$} \\
\hline & & $P$ [day $]$ & FAP & $P$ [day $]$ & FAP & $P$ [day $]$ & FAP & $P$ [day $]$ & FAP \\
\hline HD 352 & & 然 & $2.92 \times 10^{-3}$ & 3.59 & $1.85 \times 10^{-3}$ & $03 \pm 0.006$ & $1.25 \times 10^{-3}$ & $3.620 \pm 0.008$ & $6.87 \times 10^{-2}$ \\
\hline HD 37394 & A. 2 & $10.75 \pm 0.04$ & $7.57 \times 10^{-8}$ & $10.77 \pm 0.05$ & $4.36 \times 10^{-4}$ & $10.81 \pm 0.05$ & $6.43 \times 10^{-5}$ & $10.81 \pm 0.06$ & $1.49 \times 10^{-2}$ \\
\hline HD 39587 & A. 3 & $5.13 \pm 0.01$ & $7.50 \times 10^{-5}$ & $5.39 \pm 0.01$ & $6.99 \times 10^{-3}$ & $5.60 \pm 0.01$ & $2.21 \times 10^{-3}$ & $5.34 \pm 0.01$ & $1.99 \times 10^{-4}$ \\
\hline HD 43162 & A. 4 & $7.09 \pm 0.02$ & $1.40 \times 10^{-4}$ & $7.09 \pm 0.02$ & $1.11 \times 10^{-3}$ & $7.10 \pm 0.02$ & $4.27 \times 10^{-5}$ & $7.11 \pm 0.02$ & $1.12 \times 10^{-5}$ \\
\hline HD 72905 & A. 5 & $5.22 \pm 0.01$ & $4.43 \times 10^{-3}$ & $5.23 \pm 0.01$ & $8.08 \times 10^{-4}$ & $5.23 \pm 0.02$ & $1.96 \times 10^{-3}$ & $5.22 \pm 0.01$ & $7.40 \times 10^{-5}$ \\
\hline HD 75732 & A. 6 & $37.4 \pm 0.5$ & $7.50 \times 10^{-6}$ & & & & & & \\
\hline HD 97334 & A. 7 & $7.92 \pm 0.05$ & $1.61 \times 10^{-4}$ & $8.26 \pm 0.05$ & & 0.05 & $7.57 \times 10^{-5}$ & $8.15 \pm 0.05$ & $2.09 \times 10^{-3}$ \\
\hline HD 101501 & A. 8 & $17.00 \pm 0.14$ & $2.93 \times 10^{-6}$ & $16.70 \pm 0.16$ & $8.40 \times 10^{-3}$ & $15.81 \pm 0.08$ & $7.38 \times 10^{-6}$ & $15.90 \pm 0.09$ & $6.19 \times 10^{-6}$ \\
\hline HD 111395 & A. 9 & $16.3 \pm 0.2$ & $5.53 \times 10^{-7}$ & $16.2 \pm 0.2$ & $2.43 \times 10^{-5}$ & $16.0 \pm 0.1$ & $2.32 \times 10^{-6}$ & $16.2 \pm 0.2$ & $1.69 \times 10^{-3}$ \\
\hline HD 115043 & A. 10 & $5.86 \pm 0.01$ & $5.07 \times 10^{-5}$ & $5.88 \pm 0.02$ & $3.64 \times 10^{-3}$ & $5.90 \pm 0.01$ & $6.28 \times 10^{-6}$ & $5.84 \pm 0.02$ & $1.18 \times 10^{-3}$ \\
\hline HD 118972 & A.11 & $9.46 \pm 0.05$ & $5.66 \times 10^{-5}$ & $9.37 \pm 0.10$ & $2.00 \times 10^{-1}$ & $9.38 \pm 0.09$ & $2.85 \times 10^{-2}$ & $9.40 \pm 0.03$ & $4.01 \times 10^{-8}$ \\
\hline HD 131977 & A. 12 & $33.0 \pm 0.5$ & $3.43 \times 10^{-6}$ & $32.8 \pm 0.5$ & $8.93 \times 10^{-5}$ & $32.2 \pm 0.4$ & $3.01 \times 10^{-6}$ & $31.9 \pm 0.4$ & $3.32 \times 10^{-6}$ \\
\hline
\end{tabular}

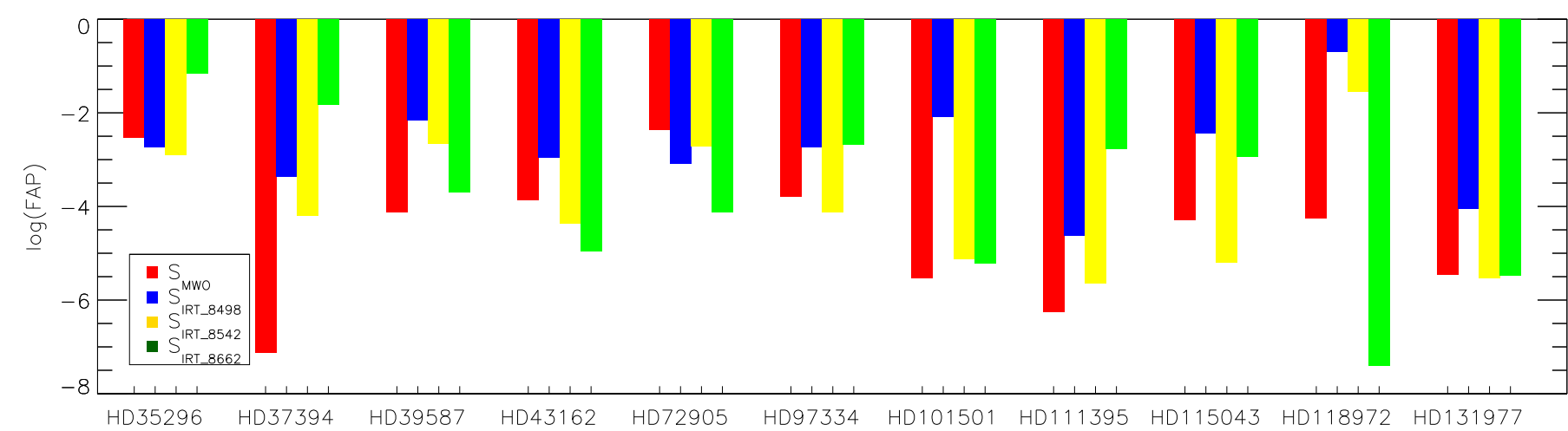

Fig. 10. Logarithm of the estimated FAPs of the derived periods in the individual time series vs. objects. The red bars show the log(FAP) values for the period derived from the $S_{\text {Mwo }}$ time series, the blue bars that from the $S_{\text {IRT_8498 }}$ time series, the yellow bars that from the $S_{\text {IRT_8542 }}$ and the green bars that from the $S_{\text {IRT_8662 }}$ time series.

\section{Results of the rotation period measurement}

In the following, we present our results of the measurement of the stellar rotation period for the $\mathrm{Ca}$ II $\mathrm{H} \& \mathrm{~K}$ lines and the Ca II IRT lines as described in Sect. 4.

\subsection{Time series for individual lines}

The results from the analysis of the time series using individual lines are listed in Table 5. Each step in the process of rotation period determination for each of the 12 studied stars is documented in different graphs found in Figs. A.1 to A.12, including the raw time series of the indices with the used fit for the detrending, determined periods as a function of the order of the detrending polynomial, periodograms, and phase-folded time series of the detrended indices.

For 11 out of 12 sample objects, we find a significant rotation period with a FAP lower than $2.7 \times 10^{-3}(3 \sigma)$ in at least one of the Ca II IRT lines and a correlation between the indices; see Figs. A.1 to A.12. Only for HD 75732 do we find no period using the Ca II IRT lines and also no correlation between the indices; see Fig. A.6. Moreover, the derived period in the $S_{\text {Mwo }}$ time series of HD 75732 also strongly depends on the chosen detrending procedure; see Fig. A.6. The resulting period range is 40.5 to 37.4 days for detrending with a polynomial of the first, second, and third order. The reason for this behaviour is a long-term trend and a periodic variation with a period of $\approx 100$ days. With the used polynomial fit of the third order, the long-term trend and the 100 days variation is removed and so we obtain a 37.4 day period for HD 75732.

To compare the FAPs of the periods found in the $S_{\text {MwO }}$ and in the $S_{\text {IRT }}$ time series, the logarithm of the FAPs for each time series are shown as a bar plot vs. the objects in Fig. 10, except for HD 75732. Figure 10 shows that in only four cases is the FAP of the period derived from the $S_{\text {MWO }}$ time series the smallest FAP of all time series considered.

In Fig. 11 the periods obtained from the $S_{\text {MWO }}$ and the $S_{\text {IRT- }}$ indices are plotted. These periods follow the identity with a small deviation for some objects. To quantify this deviation, we calculate the relative difference between the period found from the $S_{\text {IRT- }}$ and $S_{\text {MWO-indices (see Fig. 11); the difference between the }}$ periods is lower than $9 \%$. The majority of the periods are identical to within the $3 \sigma$ errors. However, a few periods are clearly different. These variations of the periods are of the order of the variations of the minimal and maximal rotation periods to the mean rotation periods listed in Donahue et al. (1996, Table 1); for a possible reason see our discussion in Sect. 6 .

\subsection{Most probable rotation period}

For eleven stars, we find a rotation period in the $S_{\text {MwO }}$ time series and in the individual $S_{\text {IRT }}$ time series. To estimate the most 
M. Mittag et al.: Comparison measurement of rotation periods with Ca II H\&K and Ca II IRT

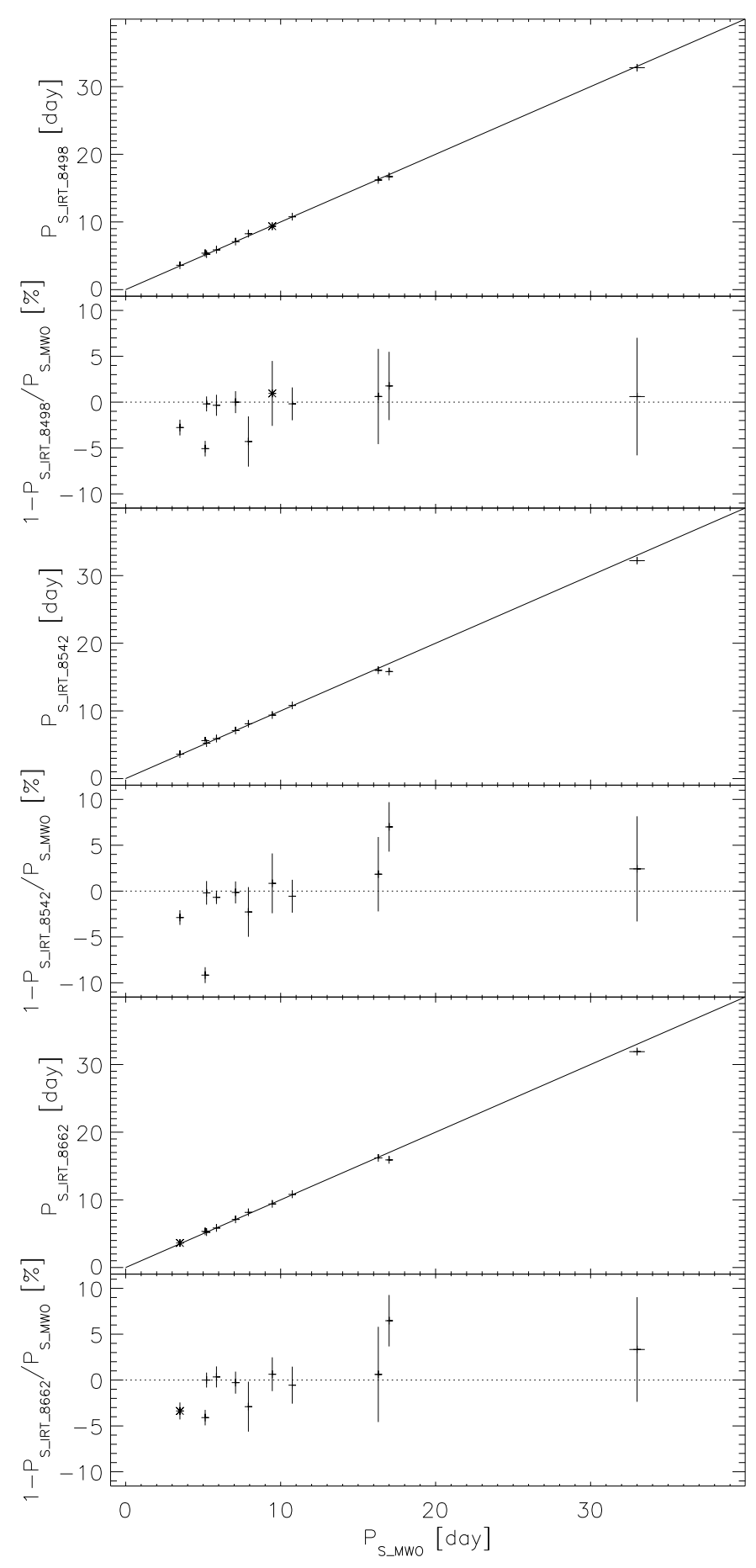

Fig. 11. Comparison of the periods derived from the $S_{\text {IRT }}$-indices time series vs. the periods derived from the $S_{\mathrm{MwO}}$ time series (in the $u p$ per panels) with the identity as solid line. The lower panels show the relative difference (in percent) between the periods derived in the $S_{\mathrm{IRT}^{-}}$ indices and $S_{\text {Mwo }}$ time series vs. the period obtained from the $S_{\text {Mwo }}$ time series with a $3 \sigma$ error for the period ratios. The periods with a FAP less than $4.55 \times 10^{-2}(2 \sigma)$ are labelled with a plus symbol and periods with a FAP greater than $4.55 \times 10^{-2}$ with an asterisk.

probable rotation period and the corresponding FAP, the periodograms are combined with the method described in Sect. 4 and shown in Figs. A.1 to A.12. Here, we use all four time series even if one time series shows a weak signal in the periodogram. The results for the eleven stars are listed in Table 6. For completeness, the determined period for HD 75732 is also listed in
Table 6. Literature period, most probable rotation period and the corresponding FAP.

\begin{tabular}{lccc}
\hline \hline Object & $P_{\text {Lit }}[$ day $]$ & $P_{\mathrm{M}}[$ day $]$ & FAP \\
\hline HD 35296 & $3.56^{2}$ & $3.604 \pm 0.004$ & $4.38 \times 10^{-6}$ \\
HD 37394 & $11.00^{3}$ & $10.78 \pm 0.02$ & $1.32 \times 10^{-6}$ \\
HD 39587 & $5.36^{2}$ & $5.36 \pm 0.01$ & $2.82 \times 10^{-8}$ \\
HD 43162 & & $7.10 \pm 0.01$ & $3.84 \times 10^{-5}$ \\
HD 72905 & $4.9^{2}$ & $5.22 \pm 0.01$ & $4.55 \times 10^{-6}$ \\
HD 75732 & $42.00^{1}$ & $37.4 \pm 0.5$ & $7.50 \times 10^{-6}$ \\
HD 97334 & $7.60^{3}$ & $7.94 \pm 0.03$ & $1.61 \times 10^{-5}$ \\
HD 101501 & $16.68^{2}$ & $16.06 \pm 0.06$ & $4.53 \times 10^{-6}$ \\
HD 111395 & & $16.17 \pm 0.08$ & $7.04 \times 10^{-8}$ \\
HD 115043 & $6.0^{3}$ & $5.87 \pm 0.01$ & $3.49 \times 10^{-6}$ \\
HD 118972 & & $9.41 \pm 0.02$ & $2.87 \times 10^{-5}$ \\
HD 131977 & & $32.5 \pm 0.2$ & $1.08 \times 10^{-6}$ \\
\hline
\end{tabular}

Notes. For HD 75732 the period $\left(P_{\mathrm{M}}\right)$ is only from the $S_{\mathrm{Mwo}}$ time series.

References. (1) Baliunas et al. (1998); (2) Donahue et al. (1996); (3) Wright et al. (2011).

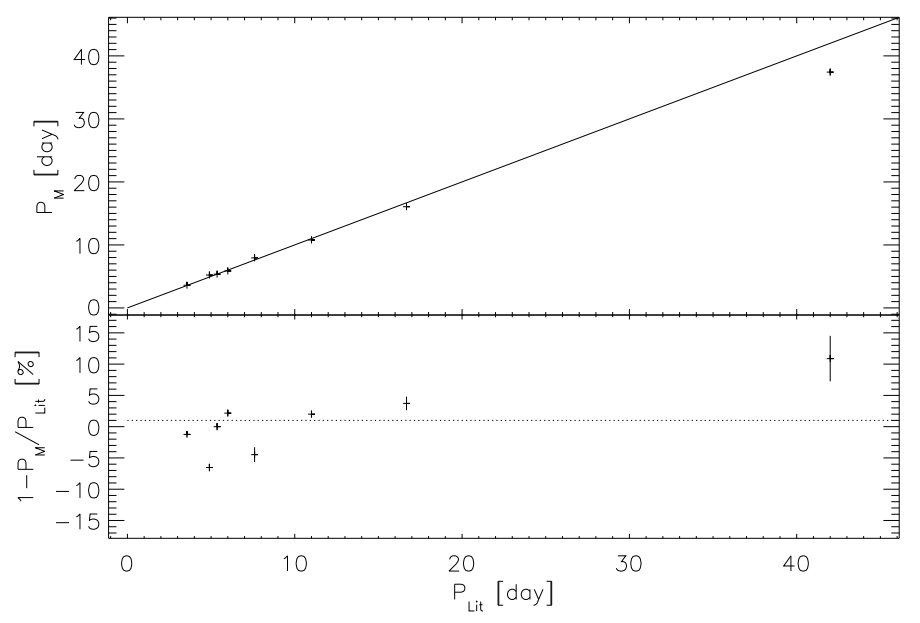

Fig. 12. Upper plot: comparison of our most probable rotation periods with the literature values with the identity as solid line. Lower panel: the relative difference in percent between our most probable rotation periods and the literature values with $3 \sigma$ error for the period ratios.

Table 6 although this period is only apparent in the $S_{\text {Mwo }}$ time series.

Finally, we compare our most probable periods with periods found in the literature (cf., see Fig. 12 and Table 6), which is the case for eight stars. For six stars, our periods agree with the literature values to within 5\%. For HD 72905 and for HD 75732 the difference between our period and the literature value is $\approx 7 \%$ and $\approx 11 \%$, respectively. The differences between ours and the literature values may be explained with the possible differential rotation of the star, as is shown by stars listed in Donahue et al. (1996, Table 1). Furthermore, we have no information about the uncertainty of the periods for the stars with a literature period from Baliunas et al. (1998) and Wright et al. (2011). Therefore, we cannot assess the true difference between these values. For HD 75732, the large discrepancy between ours and the literature value may be explained by a detrending effect; see Sect. 5.1. 


\section{Evidence for differential rotation?}

The measurement of the rotation period using spectral line variations is only possible if the star exhibits a stable distribution of spots and/or active regions on a time-scale comparable with the length of the time-series with a lifetime longer than the rotation period itself. These stellar active regions can evolve with time so that one may measure different periods at different epochs if the star rotates differentially and the active regions dominating the overall activity signal change their latitudes. This effect can be directly observed for the Sun (e.g. Hempelmann \& Donahue 1997), while for stars the situation is far more elusive, since their surfaces cannot be spatially resolved with activity indices measurements. Nevertheless, there have been several studies of differential rotation for stars using this method; for example, Donahue et al. (1996) interpret the variations of the rotation periods measured with their $S_{\text {MWO }}$ time series as a measurement of differential rotation. We note that in our sample of twelve stars (see Table 5), four stars are also included in the study of Donahue et al. (1996); namely HD 35296, HD 39587, HD 72905 and HD 101501.

In our case, we measure the rotation period only at one epoch, which should lead to the same rotation period measured in the $S_{\text {MWO }}$ and in the $S_{\text {IRT }}$ time series. However, for the stars HD 35296, HD 39587, HD 97334, and HD 101501, we actually find different rotation periods using the different indices. An inspection of the periodograms of the stars HD 35296 and HD 39587 (shown in Figs. A.1 and A.3) shows a complicated structure with multiple peaks, but very similar for the different activity indices - the only difference being the location of the highest peak. Another case, possibly indicative of differential rotation, is that of HD 101501 (Fig. A.8), which shows very broad peaks in the periodograms of the different indices, some of which even show some structure; for example, the $S_{\text {IRT_8662 }}$ time series shows a small bump at a period of $\approx 17$ days, which corresponds to the main peak found in the $S_{\text {MwO }}$ time series. Our rotation periods for the stars HD 35296 and HD 39587 determined with the individual indices fall within the minimum and maximum period found by Donahue et al. (1996) for their different data epochs, while for the star HD 101501 we find a slightly lower period than the minimum period by Donahue et al. (1996).

To check the significance of the different periods we carry out a "pre-whitening" analysis by removing our most probable period (see Table 6, Col. 3) from the time series and repeating the GLS analysis on these pre-whitened data for HD 35296, HD 39587, HD 97334 and HD 101501. Here, we test whether the peak structures are caused by aliasing or not. In the following we discuss the results for these four stars.

HD 35296: the results for the star HD 35296 are shown in Figs. A.13 and A.14 for the four indices. In the periodograms of the pre-whitened time series, no significant power peaks remain, which suggests that the complicated power distribution of the "raw" time series can be explained by aliasing effects.

HD 39587: in Fig. A.15, the periodograms of the prewhitening analysis of the 5.36 days removal are shown for the four indices and additionally in Fig. 13 for the $S_{\text {MwO }}$ and $S_{\text {IRT_8542 }}$ time series. These figures show that the peaks are generally removed, except for the $S_{\text {IRT_8542 }}$ time series. Here, the peak at 5.6 day is significantly, but not completely reduced. At this point, it is worth mentioning that the most probable period is the mean of the 5.13 and 5.6 days and therefore, these signals could be averaged out so that in the pre-whitening analysis the peaks are also removed. Hence, the pre-whitening analysis is also performed for the periods found in the $S_{\text {MWO }}$ time

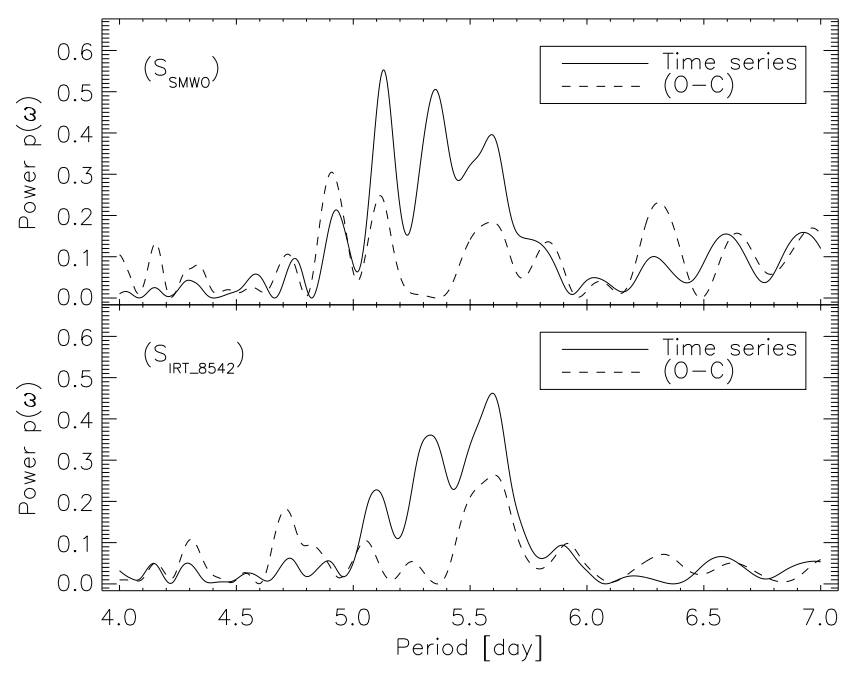

Fig. 13. Periodograms for HD 39587 of the $S_{\text {MWO }}$ and $S_{\text {IRT_8542 }}$ time series without the removal of the 5.36 day period (depicted as solid line) and after pre-whitening (dashed line).

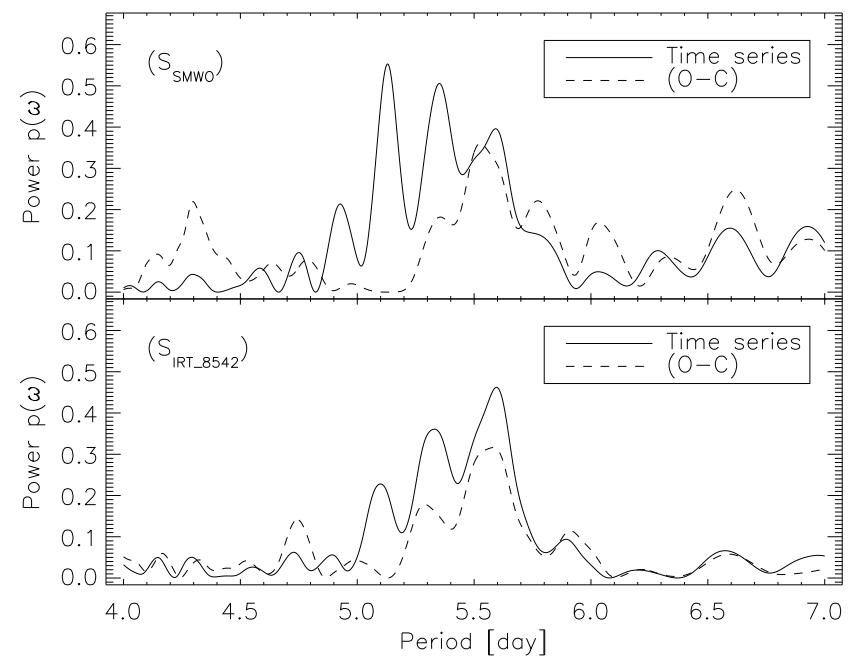

Fig. 14. Periodograms for HD 39587 of the $S_{\text {MWO }}$ and $S_{\text {IRT_8542 }}$ time series without the removal of the 5.13 day period (depicted as solid line) and after pre-whitening (dashed line).

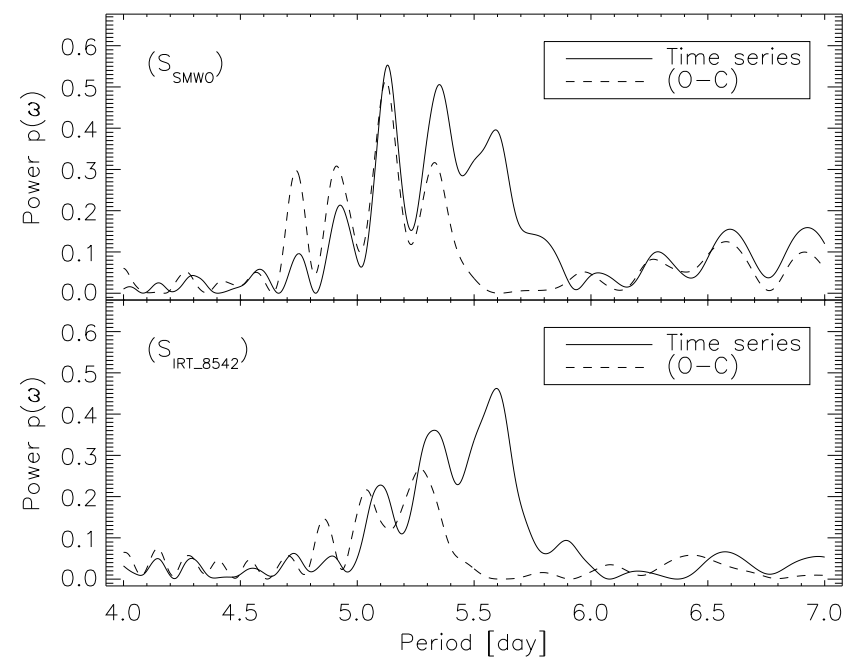

Fig. 15. Periodograms for HD 39587 of the $S_{\text {MWO }}$ and $S_{\text {IRT_8542 }}$ time series without the removal of the 5.6 day period (depicted as solid line) and after pre-whitening (dashed line). 


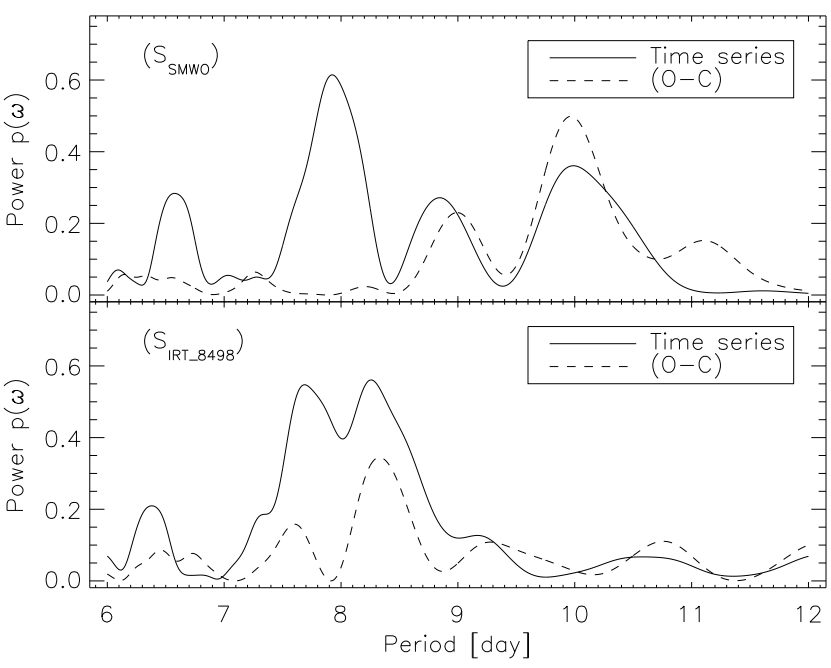

Fig. 16. Periodograms for HD 97334 of the $S_{\text {MWo }}$ and $S_{\text {IRT_8498 }}$ time series without removal of the 7.92-day period (depicted as solid line) and after pre-whitening (dashed line)

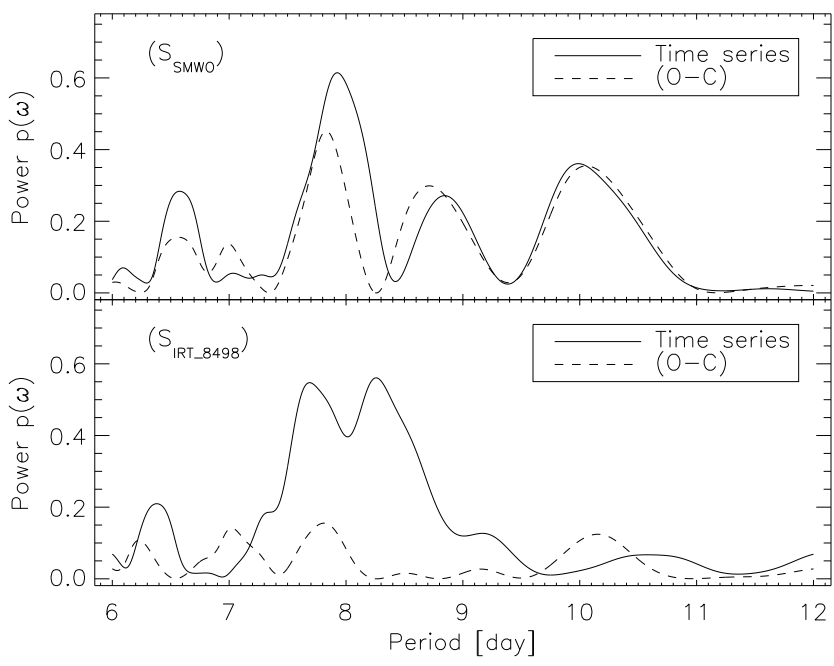

Fig. 17. Periodograms for HD 97334 of the $S_{\text {MWo }}$ and $S_{\text {IRT } 8498}$ time series without removal of the 8.26-day period (depicted as solid line) and after pre-whitening (dashed line).

series (5.13 days; see Figs. 14 and A.16) and in the $S_{\text {IRT_8542 }}$ time series (5.6 days; see Figs. 15 and A.17). We find in the case of the 5.13 days removal that the 5.6 days peak is still present in the periodograms for the $\mathrm{O}-\mathrm{C}$ data especially for the $S_{\text {MWO }}$ and $S_{\text {IRT } 8542}$ time series; see Fig. 14 . In the other case, the 5.6 day removal, the peak at the period of 5.13 days in the (O-C) periodogram for the $S_{\text {MWO }}$ time series is clearly and significantly visible. Therefore, we conclude that the periods 5.13 and 5.6 days should be real but we cannot completely exclude aliasing.

HD 97334: for the pre-whitening analysis, only the periods found in the $S_{\text {MWO }}$ and $S_{\text {IRT } 8498}$ time series are used because the most probable period is similar to the period found in the $S_{\text {Mwo }}$ time series. The results for all four time series are shown in Figs. A.18 and A.19. First, the pre-whitening analysis is performed for the period found in the $S_{\text {Mwo }}$ times series ( 7.92 day; see Figs. A.18 and 16). In this case, we find that the peak at $\approx 8.3$ days is still visible in the periodogram for the $\mathrm{O}-\mathrm{C}$ data for the $S_{\text {IRT } 8542}$ time series; see Fig. 16. For the pre-whitening analysis with the 8.26 days period (see Figs. A.19 and 17) we

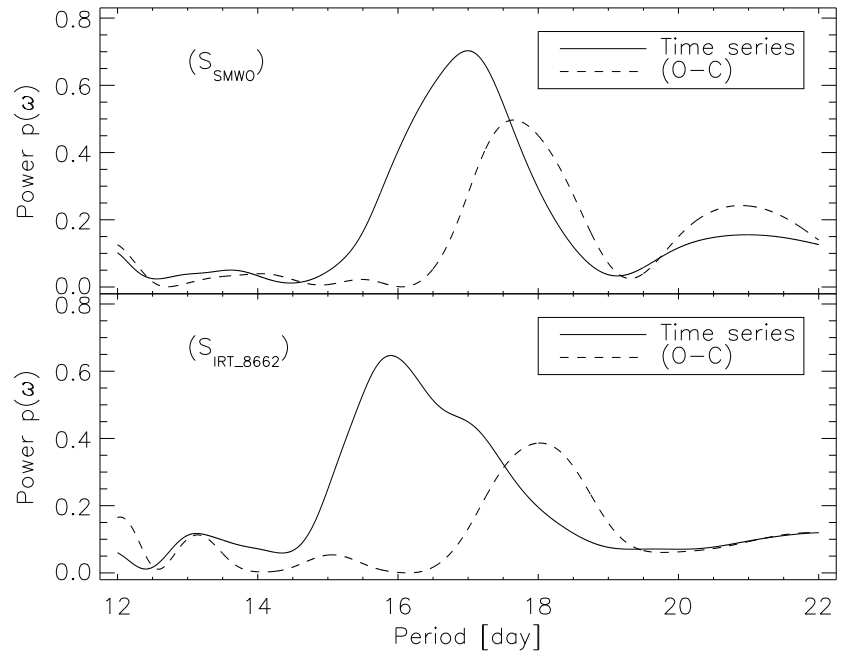

Fig. 18. Periodograms for HD 101501 of the $S_{\text {MWO }}$ and $S_{\text {IRT } 8662}$ time series without the removal of the 16.06 day period is depicted with the solid line and the data after the removal $(\mathrm{O}-\mathrm{C})$ with a dashed line.

find a peak at $\approx 7.8$ days in the periodogram for the $\mathrm{O}-\mathrm{C}$ data for the $S_{\text {MWO }}$ time series, which is close to the 7.92-day period. Hence, we conclude that the difference between the periods 7.92 and 8.26 days could be real, but just like the case of HD 39587, we cannot fully exclude aliasing.

HD 101501: the periodograms of the pre-whitening analysis are shown for the four indices in Fig. A.20. For the $S_{\text {MwO }}$ time series a clear peak is visible in the periodogram after the removal of the most probable period. This peak at $\approx 17.5$ days is slightly shifted to the peak before the removal but it is clearly there. Also in the $S_{\text {IRT } 8662}$ time series this peak (at $\approx 18$ days) is visible; see Fig. 18. We conclude that this period difference between $S_{\text {MWO }}$ time series and $S_{\text {IRT_8542 }}$ and $S_{\text {IRT_8662 }}$ time series cannot be a sampling effect and therefore tentatively interpret this difference as evidence of differential rotation.

We note in this context that the excess fluxes for the Ca II H\&K and the CaII IRT lines are formed in different chromospheric layers (Montes et al. 2000). Therefore, one cannot exclude the possibility that in some cases the location of the dominant active regions, where the $\mathrm{Ca}$ II $\mathrm{H} \& \mathrm{~K}$ and the Ca II IRT lines are formed, are different and hence lead to different periods in the different lines, as suggested by the result of the prewhitening analysis for HD 101501. On the other hand, our analysis for HD 35296 (shown in Fig. A.13) demonstrates that the period differences appearing in the GLS periodograms are a sampling effect and we conclude that here more observations with a denser sampling cadence are required to arrive at conclusive results in this area of study.

\section{Discussion and conclusions}

In this study we measure rotation periods of twelve stars using the three CaII IRT lines and the simultaneously measured Ca II H\&K lines. To do so, we introduce $S_{\text {IRT-indices to }}$ characterise the excess emission in the Ca II IRT line cores and

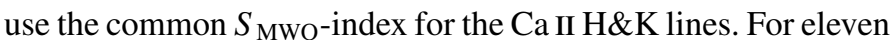
stars we find a significant rotation period at least in one of the three $S_{\text {IRT }}$ indices; the significance of these periods determined from their $S_{\text {IRT }}$ time series is comparable to the significance of the periods found in the simultaneously measured $S_{\text {MWO }}$ time series, and in a few cases is even larger. Thus we conclude that the Ca II IRT lines can indeed be used for the determination of 
the rotation period, and for the most robust period determinations one should use all the individual lines; we present a procedure to properly "add" the Lomb-Scargle periodograms obtained for different activity indices. For those stars with previous period measurements available in the literature (eight stars) we find agreement between our newly measured values and the literature values to within better than 7\%, except HD 75732 with $\approx 11 \%$.

In general, the rotation periods measured at the same epoch but in different lines are expected to be identical to within the errors and this behaviour is found in our current and previous study of HD 131977 (Mittag et al. 2015) and for six additional stars in the program stars studied here. Five stars show a somewhat different behaviour: one object shows a clear period in the $\mathrm{Ca}$ II H\&K lines, but in all Ca II IRT lines no periodic signal is visible in the periodogram; interestingly, this is the object with the lowest activity in our sample. In the other four cases, the derived GLS periodograms show a rather complicated structure. A pre-whitening for HD 35296 at the most probable period and the 3.502-day period for all four lines reduces the remaining periodograms to noise, indicating that the structures in the periodogram are due to data sampling, which we intend to improve in the future. On the other hand the result of pre-whitening the data for HD 101501 shows that the determined periods are clearly different and are not likely caused by aliasing.

In summary, we conclude that the Ca II IRT lines are usable as activity indicators and that the Ca II IRT lines are sufficiently sensitive to measure the rotation period of a star. With the possibility to use the Ca II IRT lines as activity indicators, an additional chromospheric layer (to that where the Ca II H\&K lines are formed) becomes accessible to observations.

Acknowledgements. We are grateful to our referee Dr. M. Kürster for his helpful and very constructive referee report, which led to substantial improvement of this paper. This research has made valuable use of the VizieR catalogue access tool, CDS, Strasbourg, France. The original description of the VizieR service was published in A\&AS 143, 23. We are grateful for the financial support received through the DFG project No. SCHM 1032/49-1 and we further acknowledge travel support by bilateral grants CONACyT-DFG (No.192334) and CONACyTDAAD (No. 207772) in the past 2 years, as well as by the CONACyT mobility grant (No. 207662).

\section{References}

Baliunas, S. L., Donahue, R. A., Soon, W. H., et al. 1995, ApJ, 438, 269 Baliunas, S. L., Donahue, R. A., Soon, W., \& Henry, G. W. 1998, in Cool Stars, Stellar Systems, and the Sun, eds. R. A. Donahue, \& J. A. Bookbinder, ASP Conf. Ser., 154, 153

Barnes, S. A. 2007, ApJ, 669, 1167

Donahue, R. A., Saar, S. H., \& Baliunas, S. L. 1996, ApJ, 466, 384

ESA, ed. 1997, The HIPPARCOS and TYCHO catalogues. Astrometric and photometric star catalogues derived from the ESA HIPPARCOS Space Astrometry Mission, ESA SP, 1200

Hall, J. C., \& Lockwood, G. W. 1995, ApJ, 438, 404

Hauschildt, P. H., Allard, F., \& Baron, E. 1999, ApJ, 512, 377

Hempelmann, A., \& Donahue, R. A. 1997, A\&A, 322, 835

Hempelmann, A., Mittag, M., Gonzalez-Perez, J. N., et al. 2016, A\&A, 586, A14

Horne, J. H. \& Baliunas, S. L. 1986, ApJ, 302, 757

Husser, T.-O., Wende-von Berg, S., Dreizler, S., et al. 2013, A\&A, 553, A6

Lomb, N. R. 1976, Ap\&SS, 39, 447

Martínez-Arnáiz, R., López-Santiago, J., Crespo-Chacón, I., \& Montes, D. 2011, MNRAS, 414, 2629

McQuillan, A., Mazeh, T., \& Aigrain, S. 2014, ApJS, 211, 24

Mittag, M., Schmitt, J. H. M. M., \& Schröder, K.-P. 2013, A\&A, 549, A117

Mittag, M., Hempelmann, A., Gonzalez-Perez, J. N., \& Schmitt, J. H. M. M. 2015, in Cambridge Workshop on Cool Stars, Stellar Systems, and the Sun, 18, eds. G. T. van Belle, \& H. C. Harris, 549

Mittag, M., Schröder, K.-P., Hempelmann, A., González-Pérez, J. N., \& Schmitt, J. H. M. M. 2016, A\&A, 591, A89

Montes, D., Fernández-Figueroa, M. J., De Castro, E., et al. 2000, A\&AS, 146, 103

Noyes, R. W., Hartmann, L. W., Baliunas, S. L., Duncan, D. K., \& Vaughan, A. H. 1984, ApJ, 279, 763

Piskunov, N. E., \& Valenti, J. A. 2002, A\&A, 385, 1095

Reinhold, T., Reiners, A., \& Basri, G. 2013, A\&A, 560, A4

Scargle, J. D. 1982, ApJ, 263, 835

Schmitt, J. H. M. M., Schröder, K.-P., Rauw, G., et al. 2014, Astron. Nachr., 335, 787

Vaughan, A. H., Preston, G. W., \& Wilson, O. C. 1978, PASP, 90, 267

Wright, N. J., Drake, J. J., Mamajek, E. E., \& Henry, G. W. 2011, ApJ, 743, 48

Zechmeister, M., \& Kürster, M. 2009, A\&A, 496, 577 


\section{Appendix A: Single time series}

To better illustrate our time series analysis of the different indices we show in Figs. A.1 to A.12 some graphs that represent different steps and concepts of our analysis procedure for each star. The upper four panels show the power of the highest peak, the significance of the scatter reduction compared to the nondetrended time series, the significance of the scatter reduction compared to the detrended time series with the polynomial fit of order $N-1$, and the period as a function of the order of the polynomial fit for all four used indices. The significance of the scatter reduction is estimated with the $F$-test. A detail description can be found in Sect. 4.1. The following six middle panels show the correlation between the new $S_{\text {IRT-indices and the }}$
$S_{\text {MWO-index for the original data (upper three panels) and for the }}$ data detrended with the used polynomial fit (lower three panels). For HD 101501, all time series are not detrended so that only the correlations of the original data show. After a page break, the upper four panels show the original time series of all four indices, with the black line denoting the best polynomial fit used for detrending. The middle two panels show: (left) the obtained periodogram for all four indices with the mean probability level (Prob, see Sect. 4.3) 1 $\sigma, 2 \sigma, 3 \sigma$ and $4 \sigma$; and (right) detrended and phasefolded time series for all four indices with the black line denoting the best-fitting sinusoidal. More information about the period estimation can be found in Sect. 4.2. The lower two panels show the window function of the time series on the left side and the mean periodogram of the four time series on the right side. 

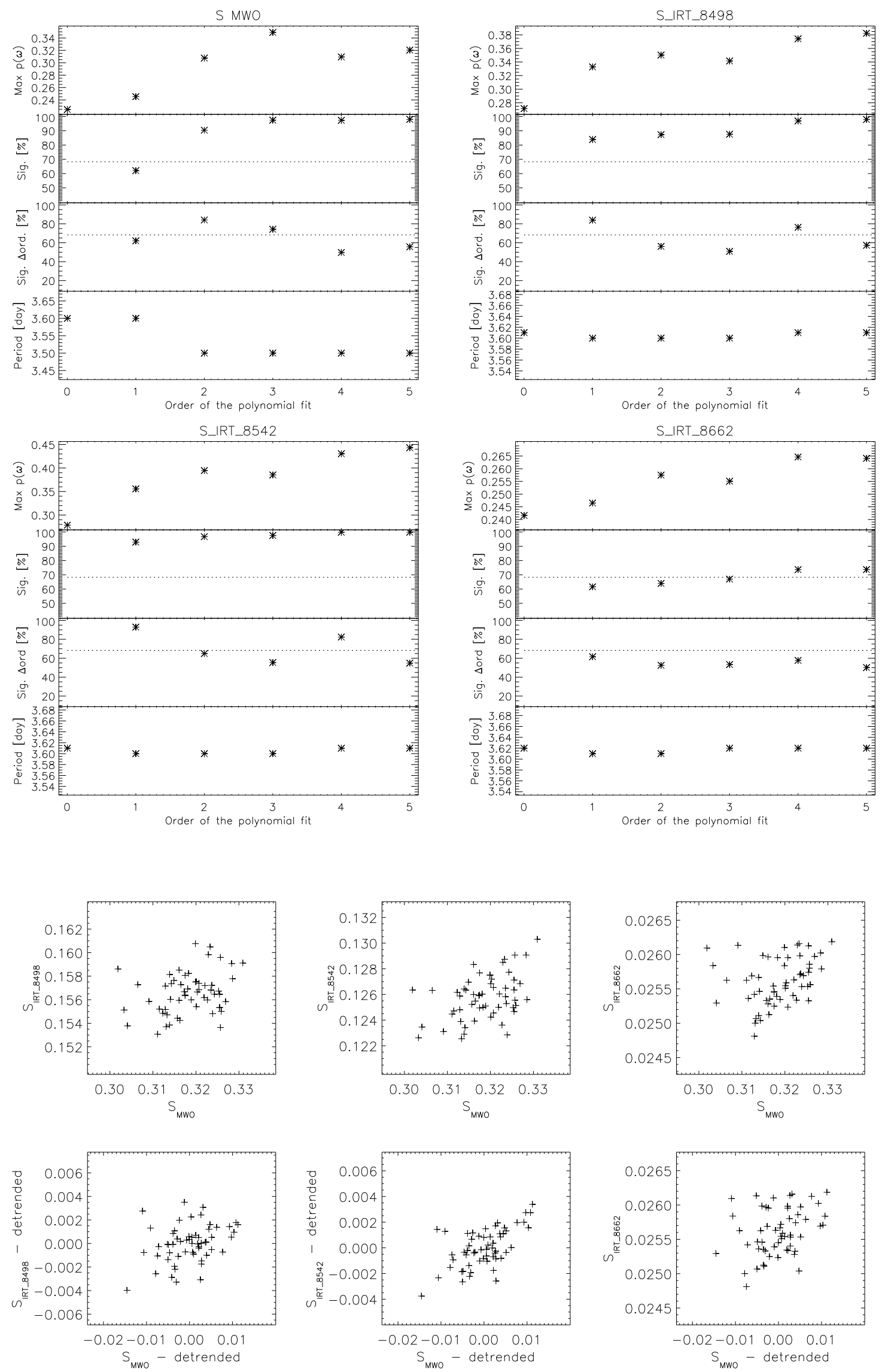

Fig. A.1. Plots for the time series analysis of HD 35296 and, for the detrending, the polynomial orders of 3, 1, 1, and 0 are used for $S_{\text {MWo }}, S_{\text {IRT } 8498}$, $S_{\text {IRT_8542 }}$, and $S_{\text {IRT_8662 }}$. A description of these figures can be found at the beginning of this Appendix. 
M. Mittag et al.: Comparison measurement of rotation periods with Ca II H\&K and Ca II IRT
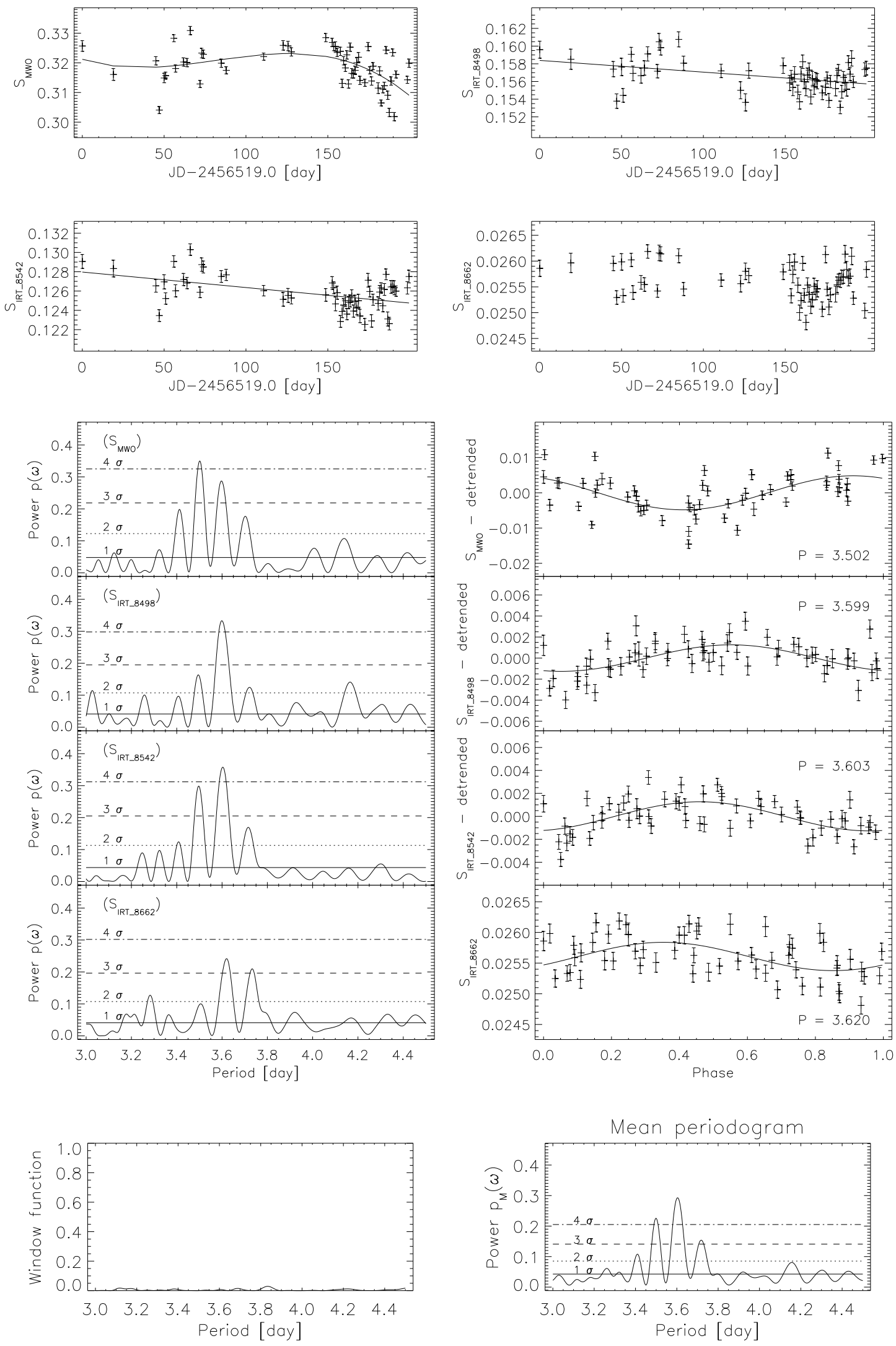

Fig. A.1. continued. 

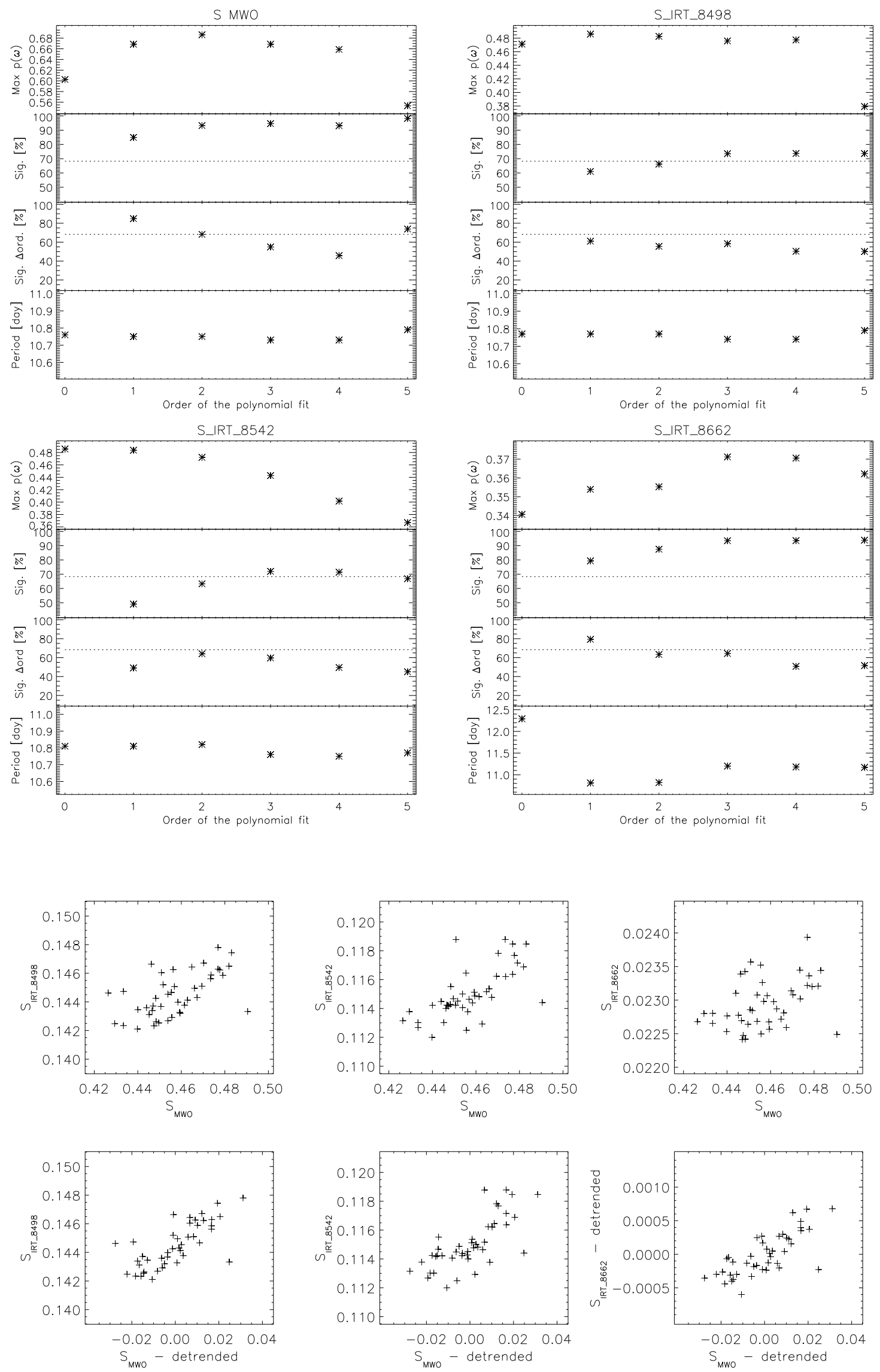

Fig. A.2. Plots for the time series analysis of HD 37394, and, for the detrending, the polynomial orders of $1,0,0$ and 1 are used for $S_{\text {MwO }}, S_{\text {IRT } \_8498}$,

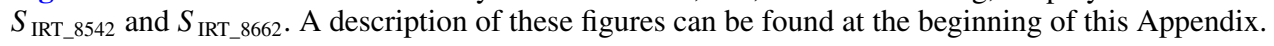


M. Mittag et al.: Comparison measurement of rotation periods with Ca II H\&K and Ca II IRT
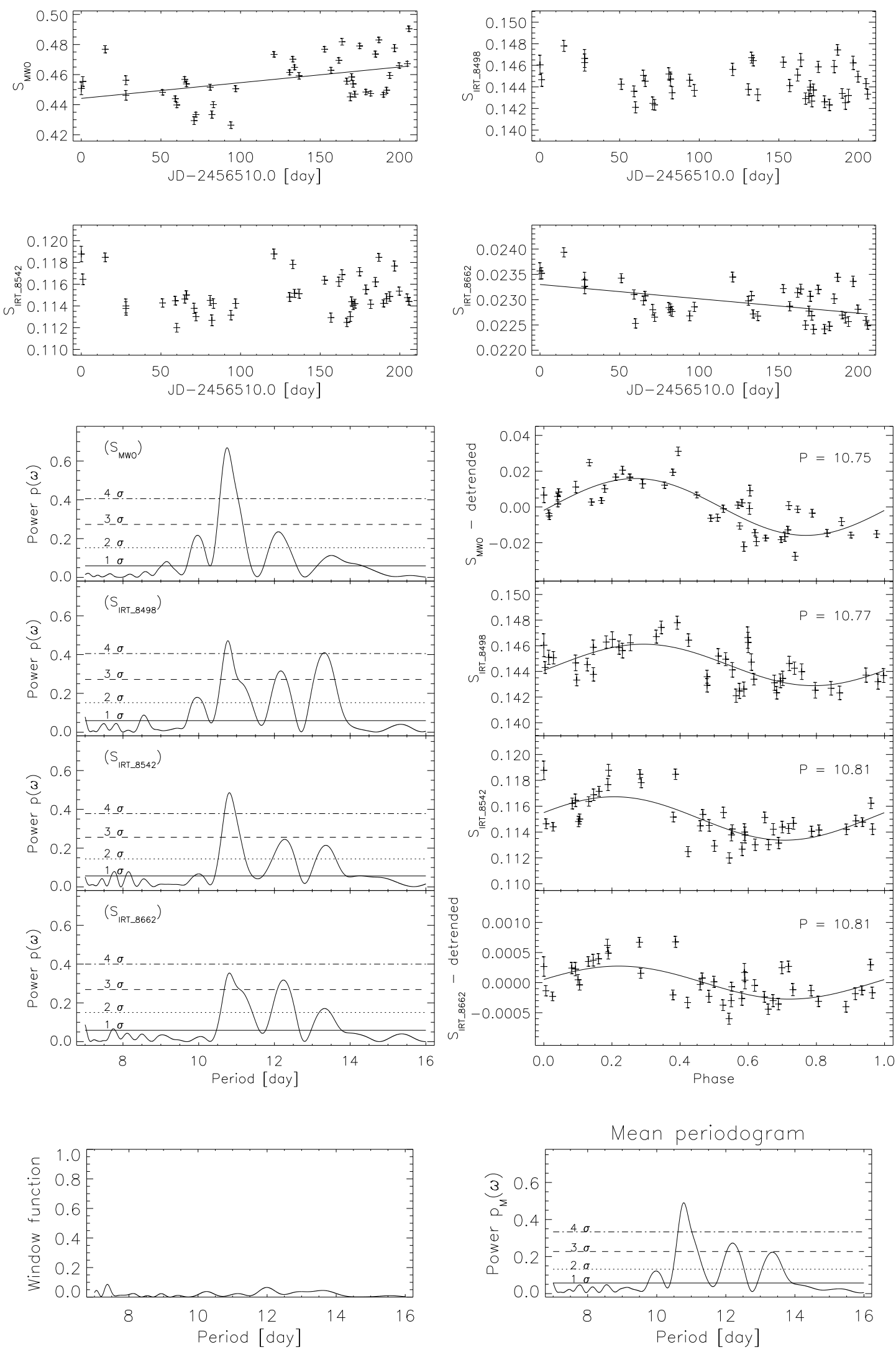

Fig. A.2. continued. 

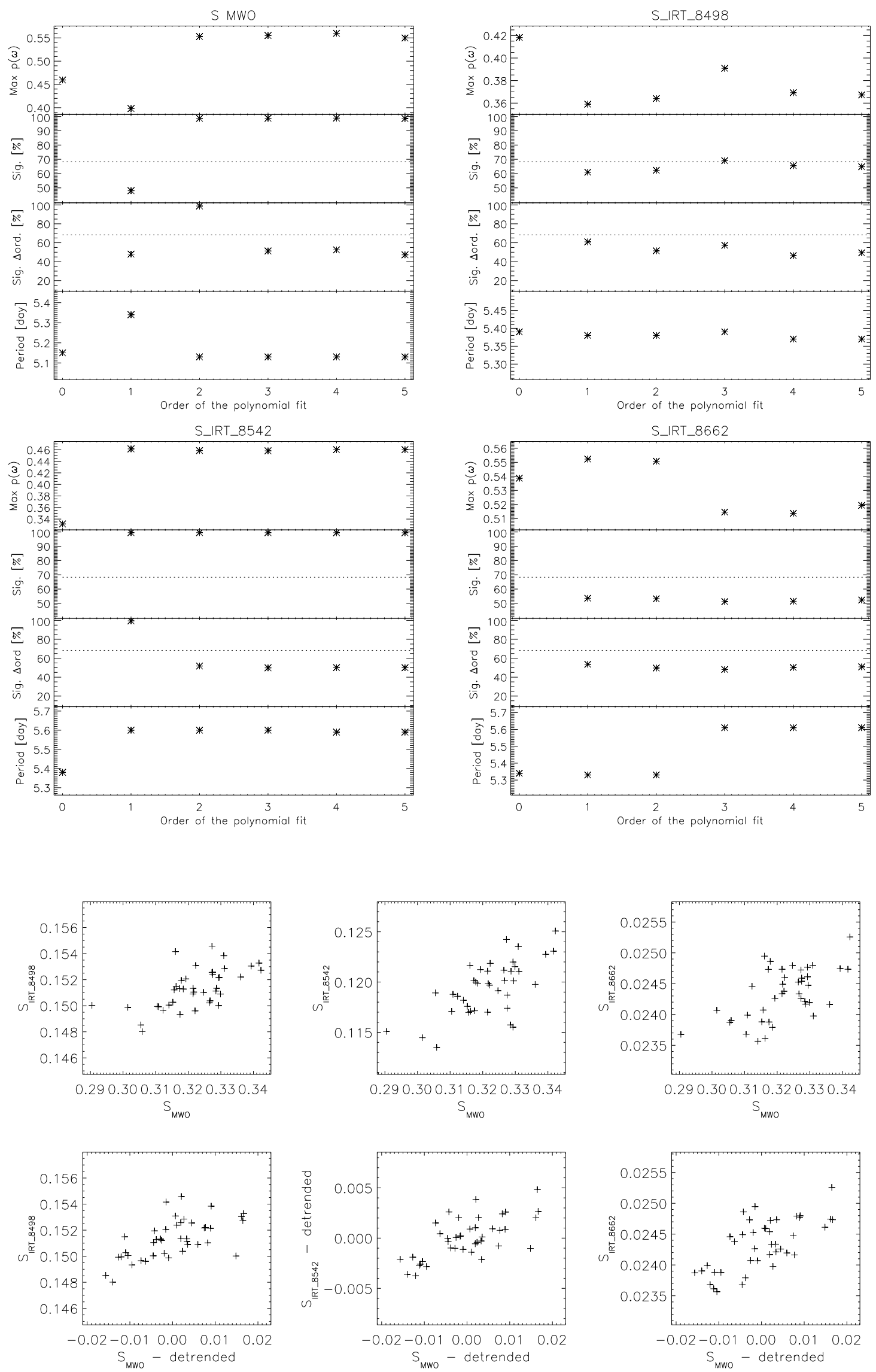

Fig. A.3. Plots for the time series analysis of HD 39587 and, for the detrending, the polynomial orders of $2,0,1$ and 0 are used for $S_{\text {Mwo }}, S_{\text {IRT } \_8498}$,

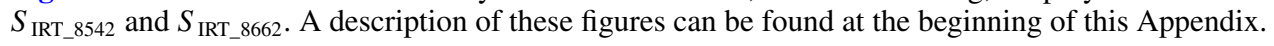


M. Mittag et al.: Comparison measurement of rotation periods with Ca II H\&K and Ca II IRT
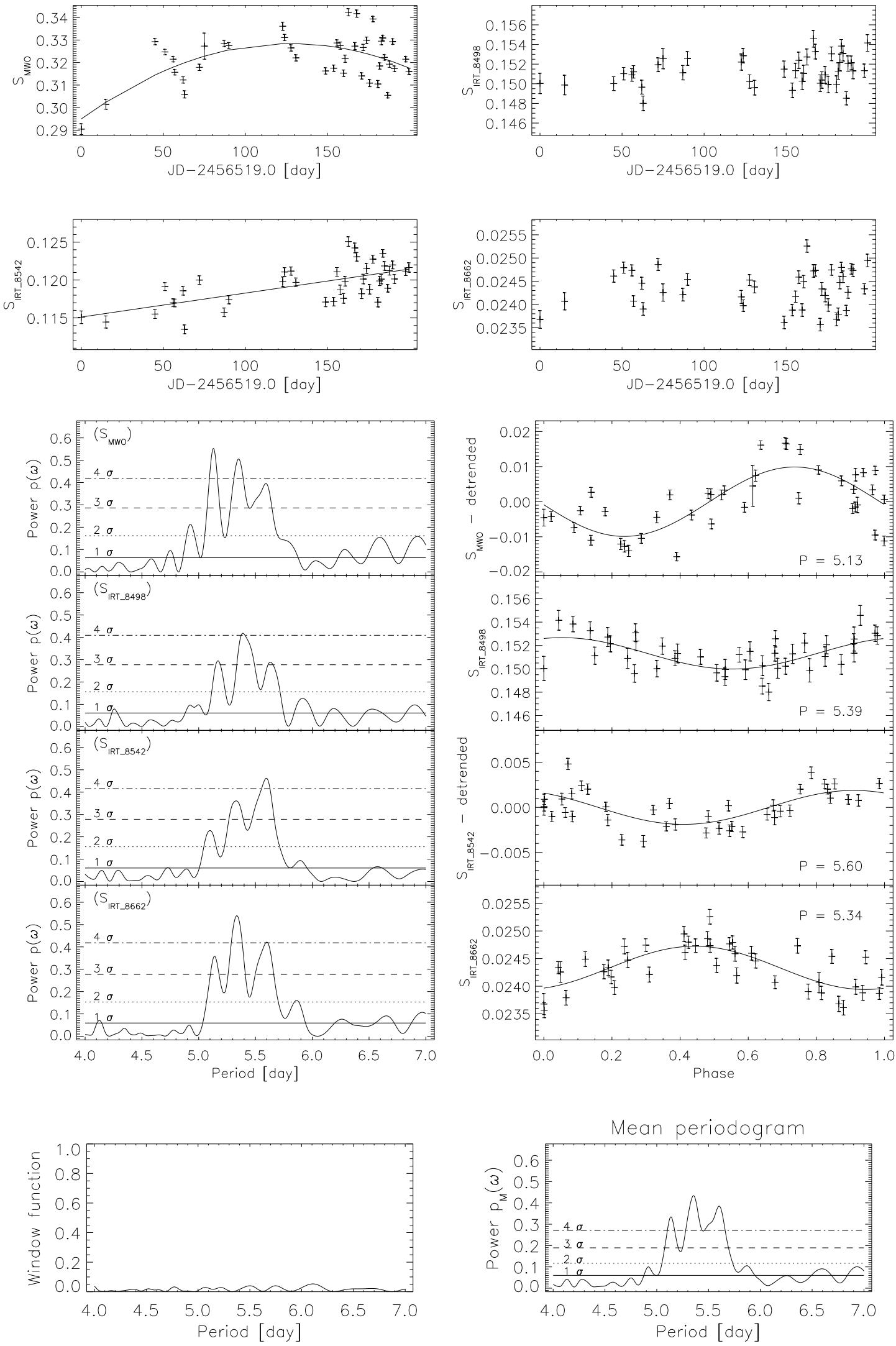

Fig. A.3. continued. 

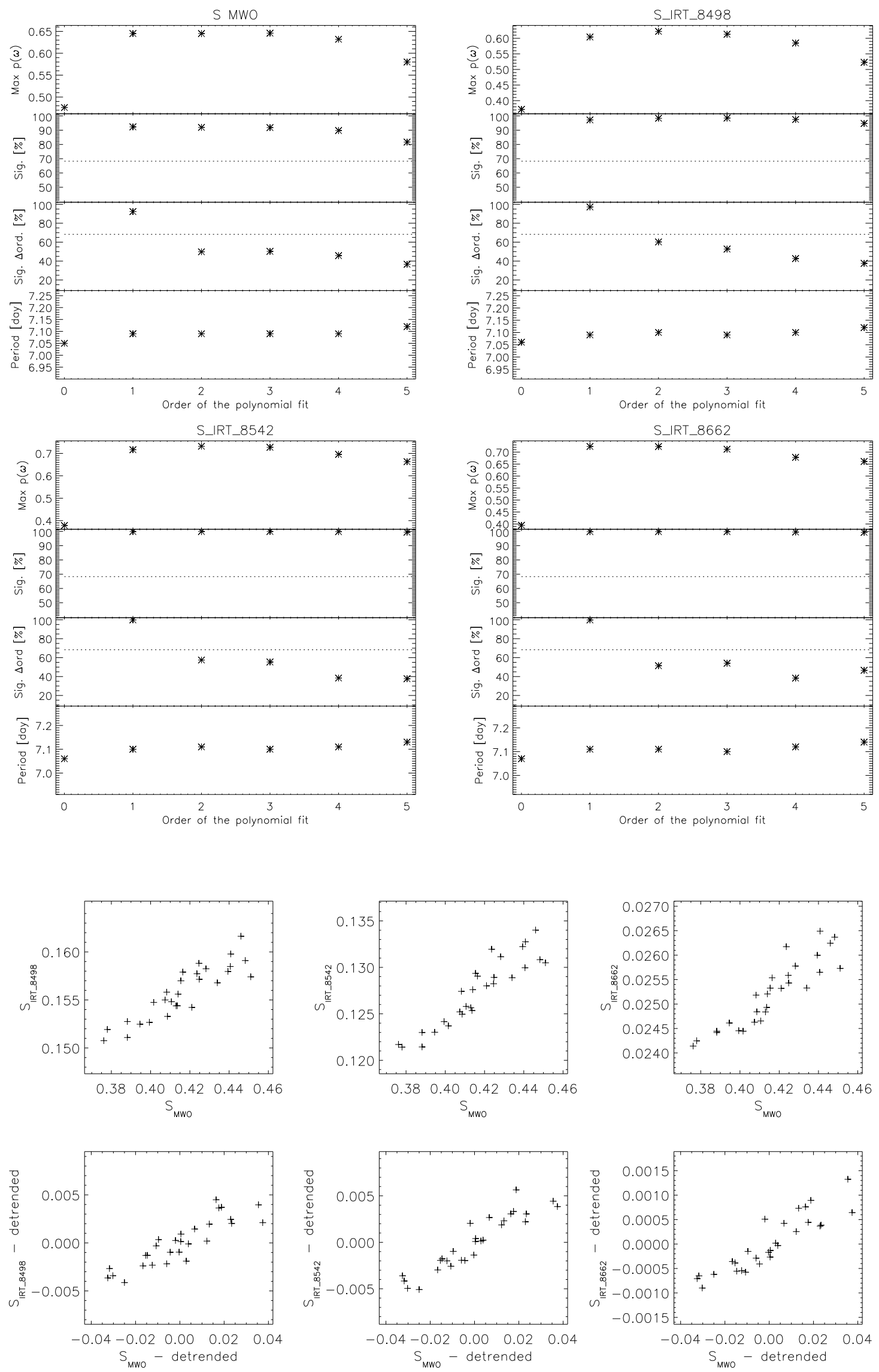

Fig. A.4. Plots for the time series analysis of HD 43162 and, for the detrending, the polynomial orders of $1,1,1$ and 1 are used for $S_{\text {Mwo }}, S_{\text {IRT } \_8498}$,

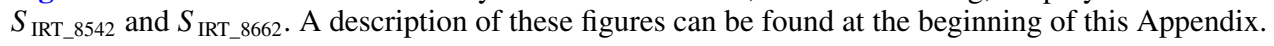


M. Mittag et al.: Comparison measurement of rotation periods with Ca II H\&K and Ca II IRT
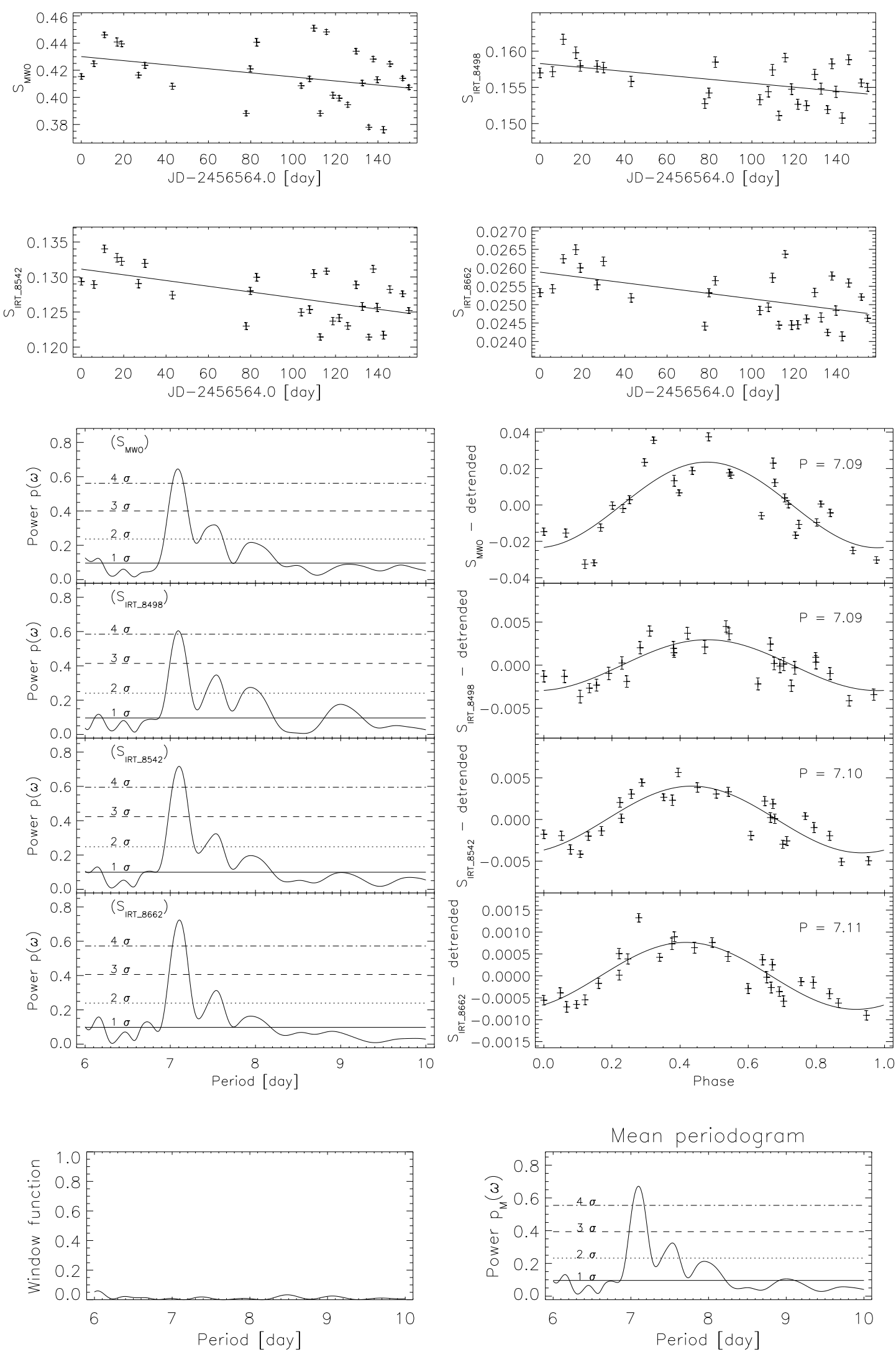

Fig. A.4. continued. 
A\&A 607, A87 (2017)
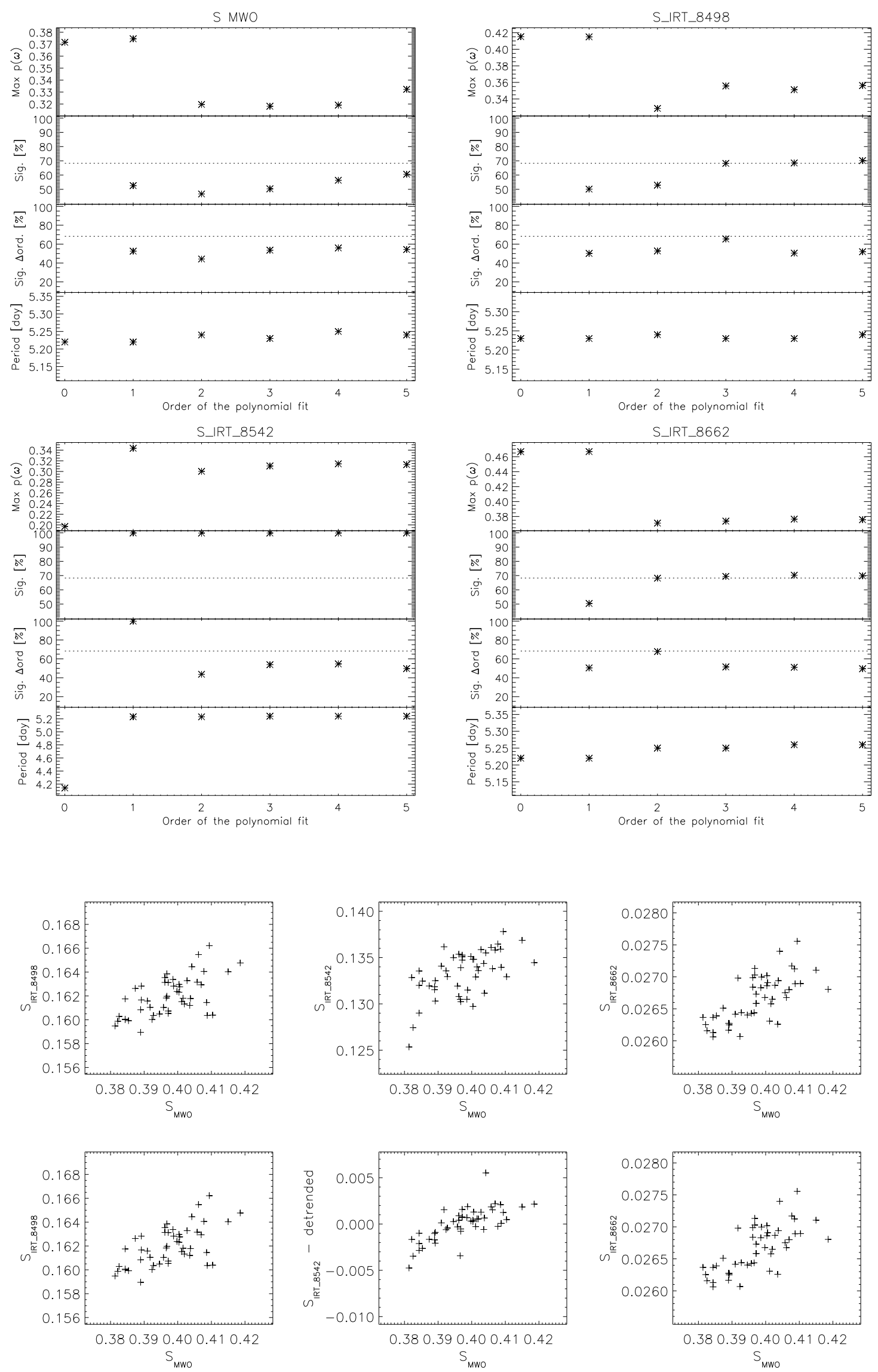

Fig. A.5. Plots for the time series analysis of HD 72905 and, for the detrending, the polynomial orders of $0,0,1$ and 0 are used for $S_{\text {Mwo }}, S_{\text {IRT } \_8498}$,

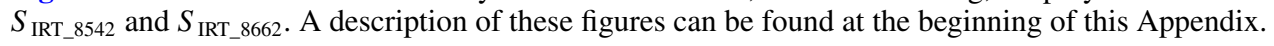


M. Mittag et al.: Comparison measurement of rotation periods with Ca II H\&K and Ca II IRT
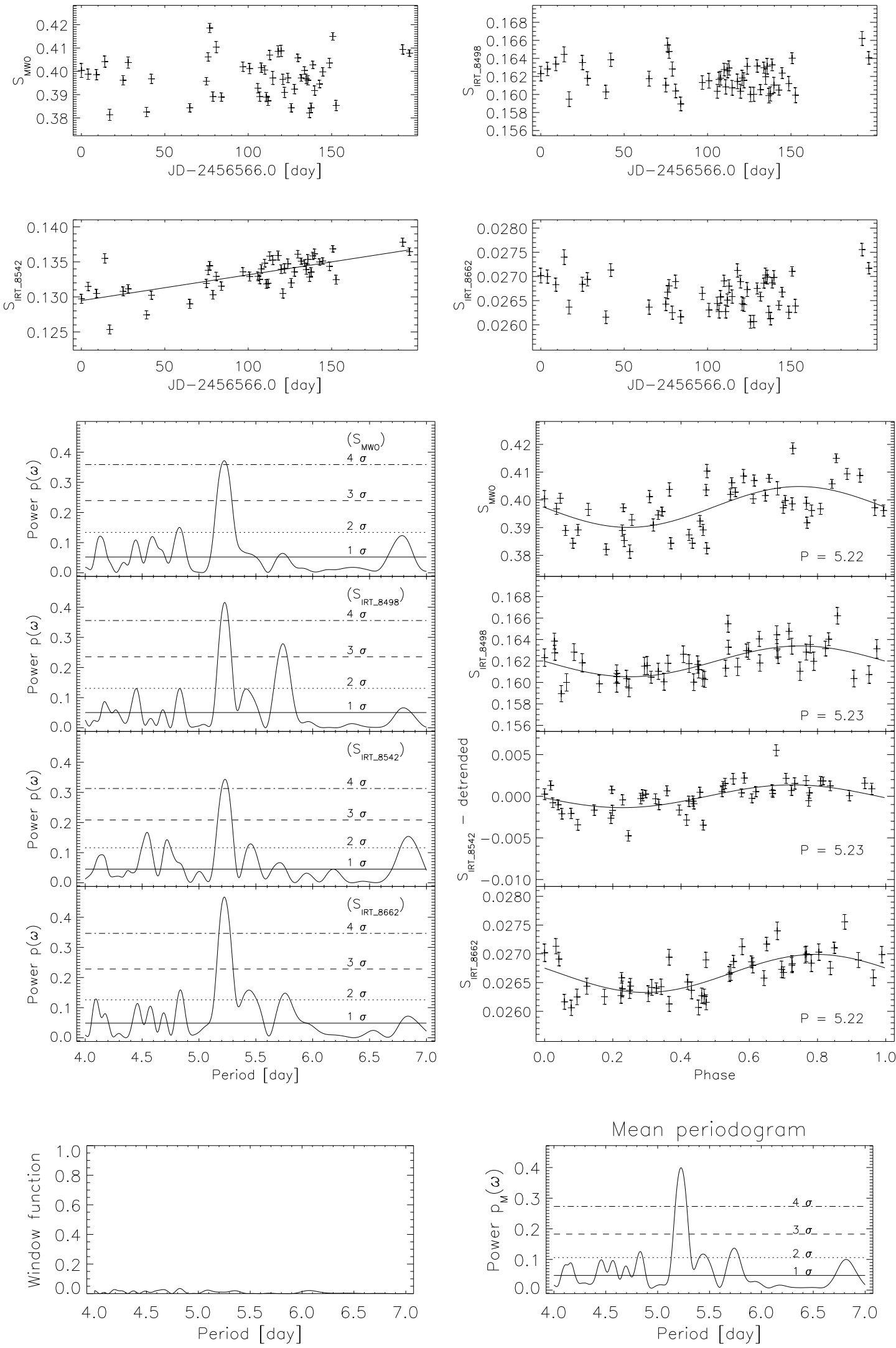

Fig. A.5. continued. 

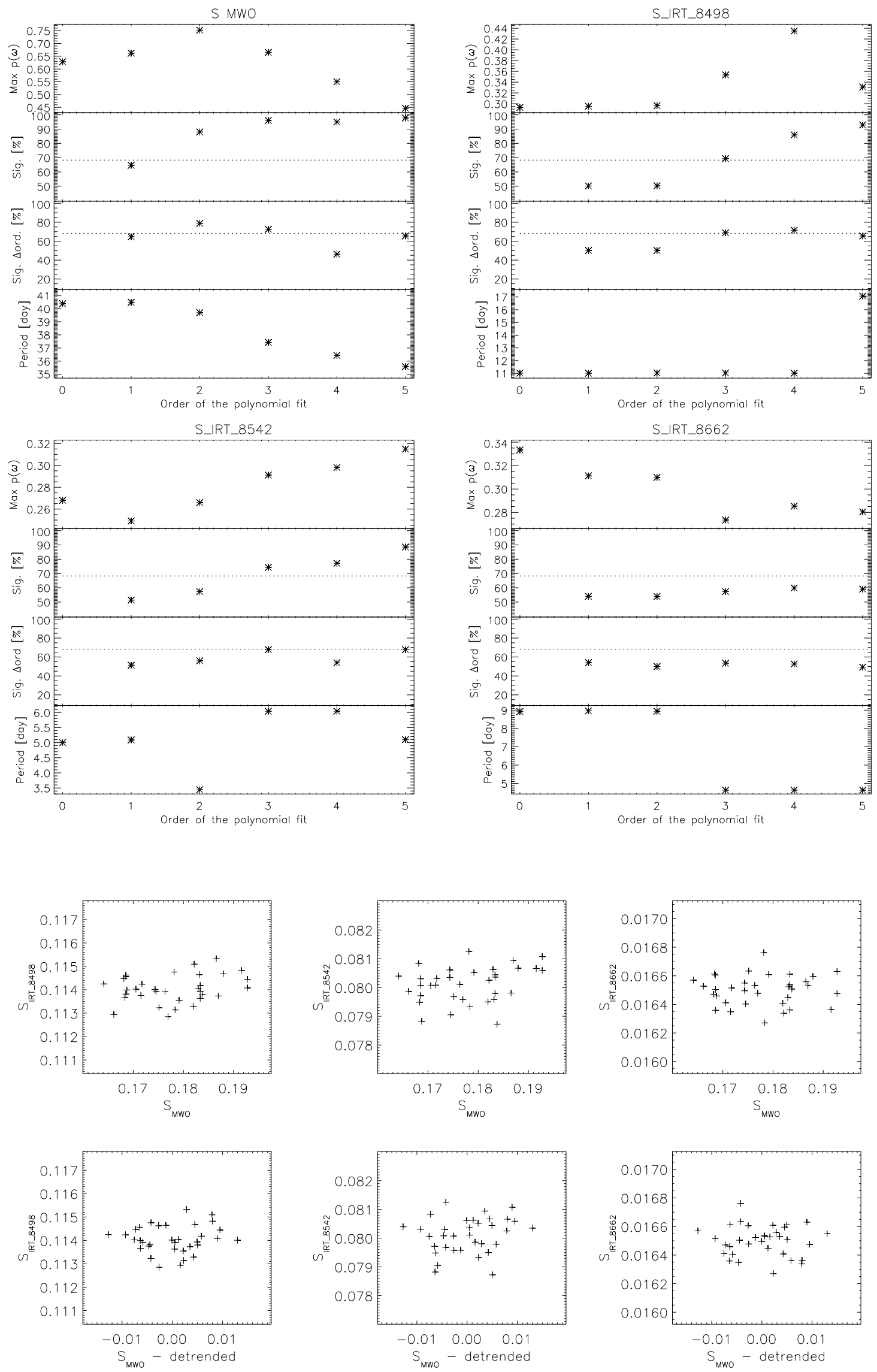

Fig. A.6. Plots for the time series analysis of HD 75732 and, for the detrending, the polynomial orders of $3,0,0$ and 0 are used for $S_{\text {Mwo }}, S_{\text {IRT } \_8498}$,

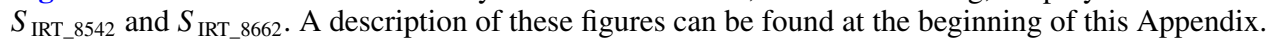


M. Mittag et al.: Comparison measurement of rotation periods with Ca II H\&K and Ca II IRT
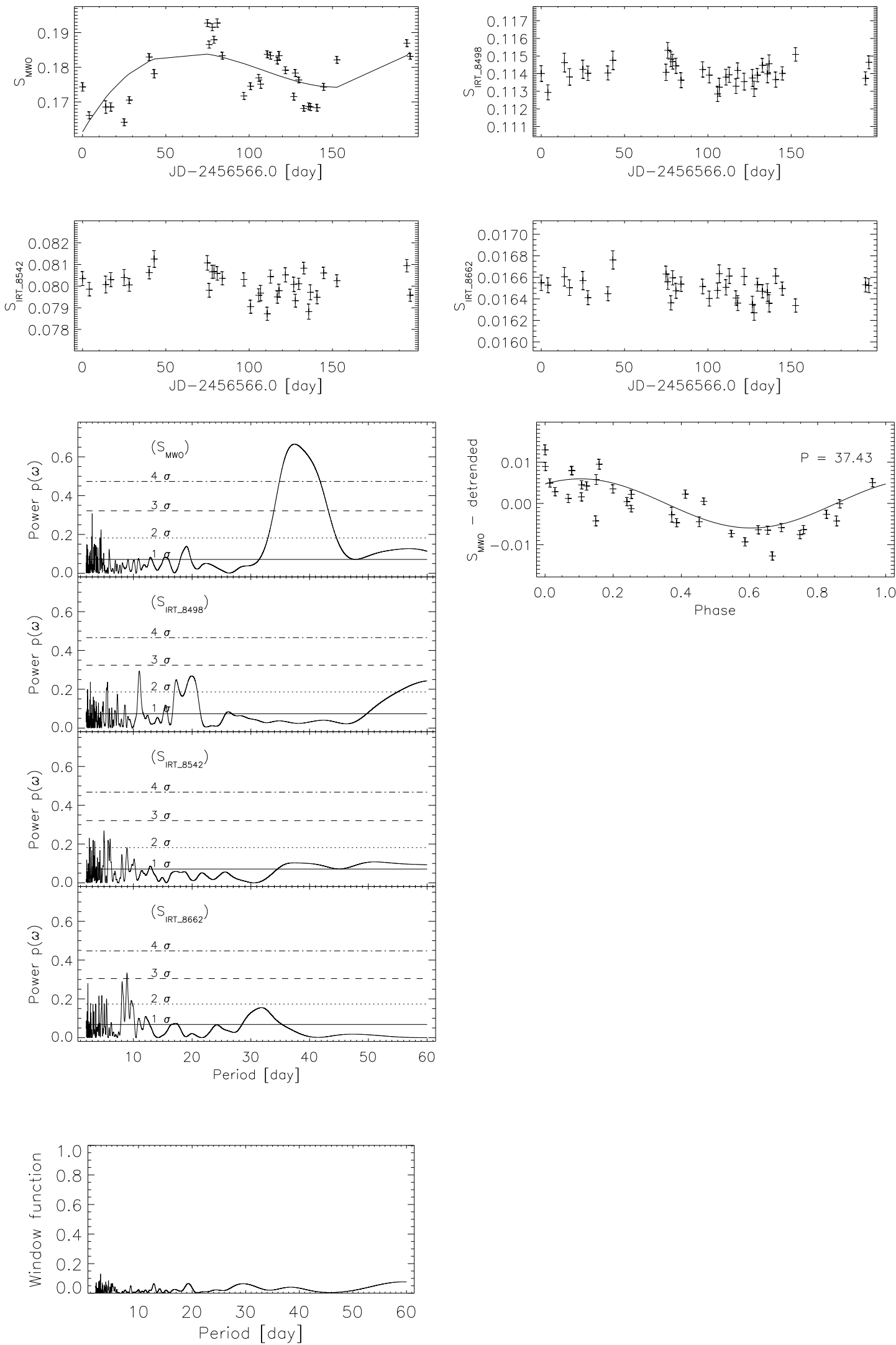

Fig. A.6. continued. 

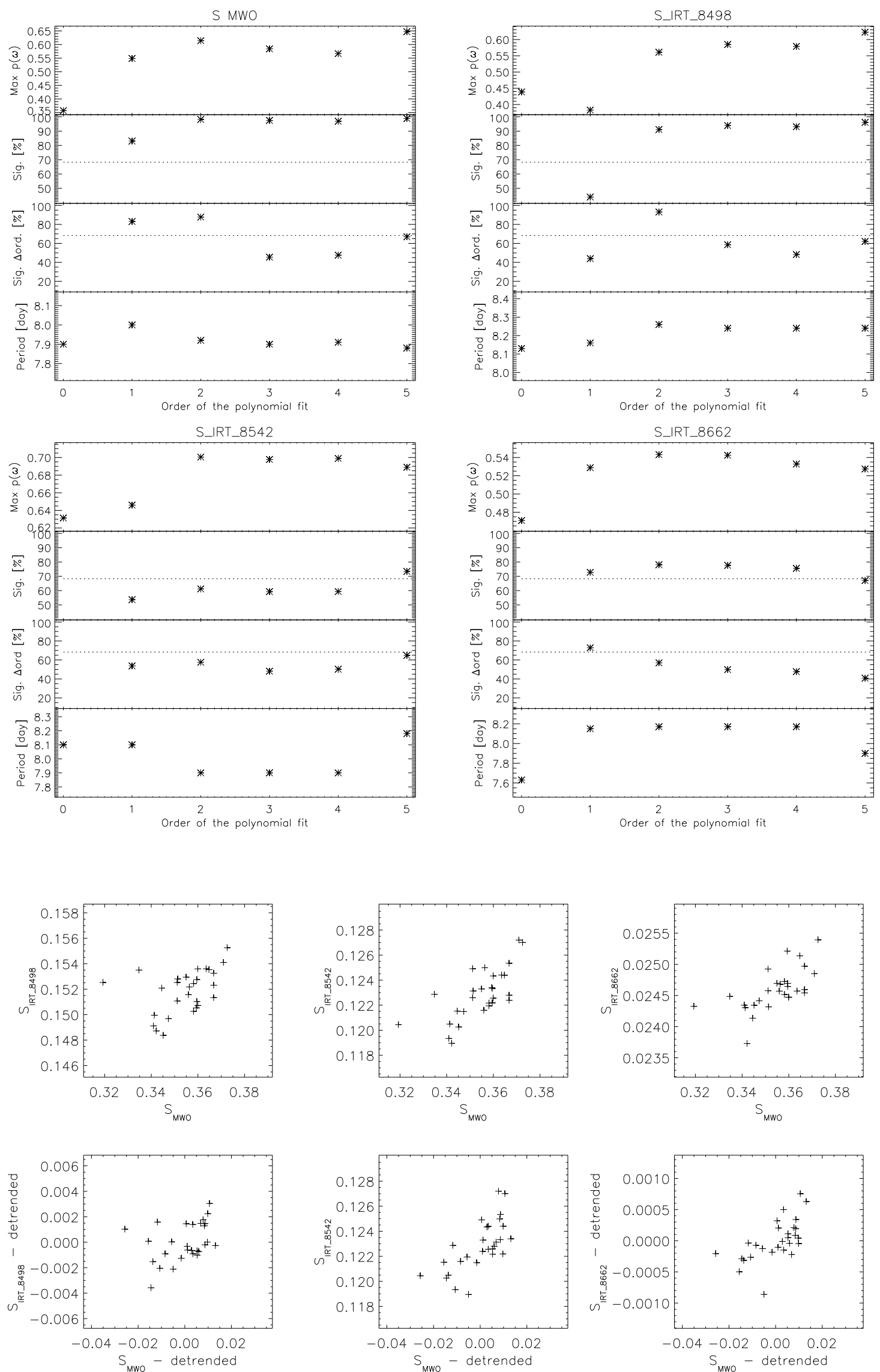

Fig. A.7. Plots for the time series analysis of HD 97334 and, for the detrending, the polynomial orders of 2, 2, 0 and 1 are used for $S_{\text {Mwo }}, S_{\text {IRT } \_8498}$,

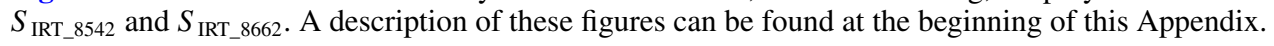


M. Mittag et al.: Comparison measurement of rotation periods with Ca II H\&K and Ca II IRT
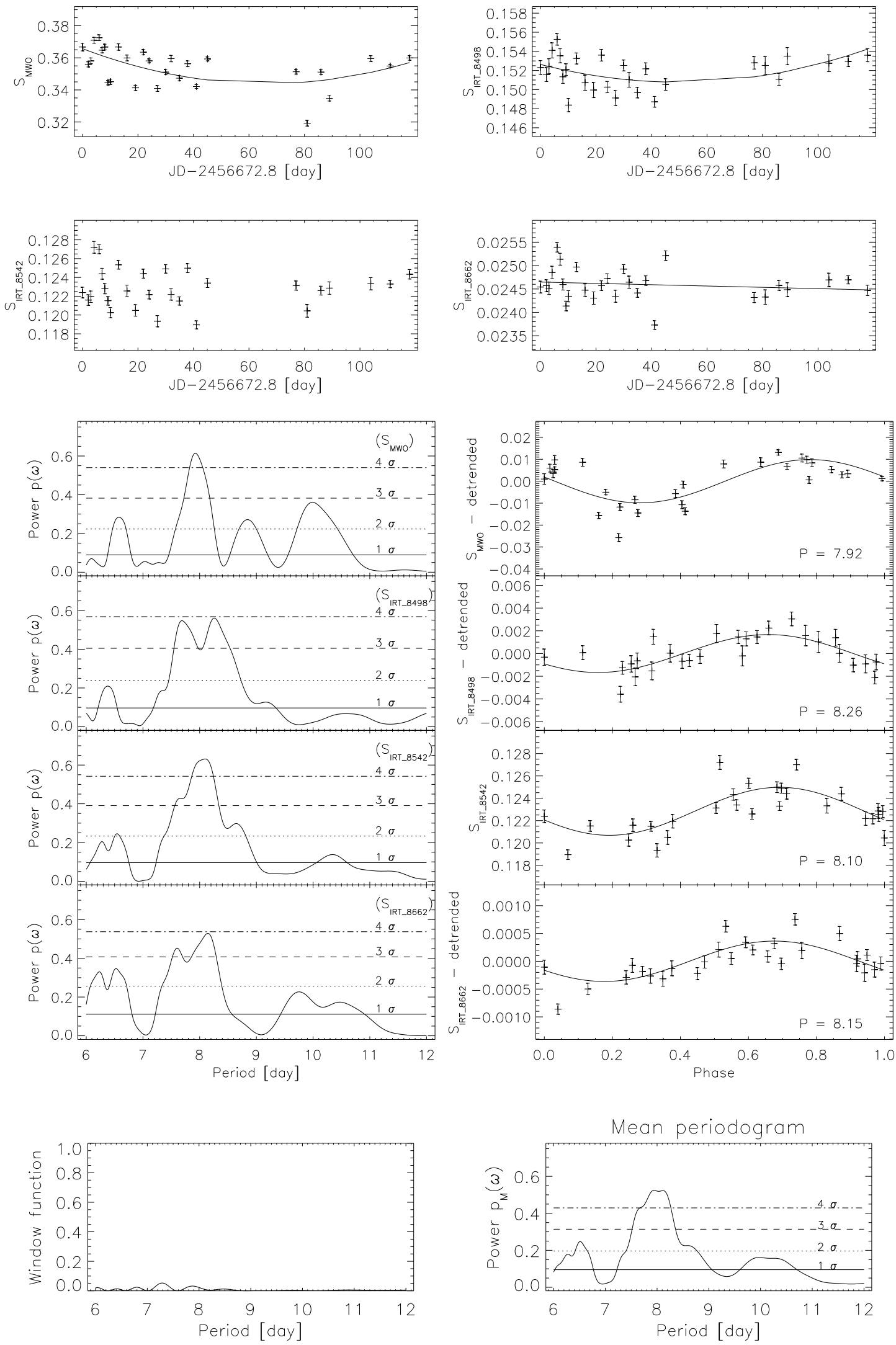

Fig. A.7. continued. 

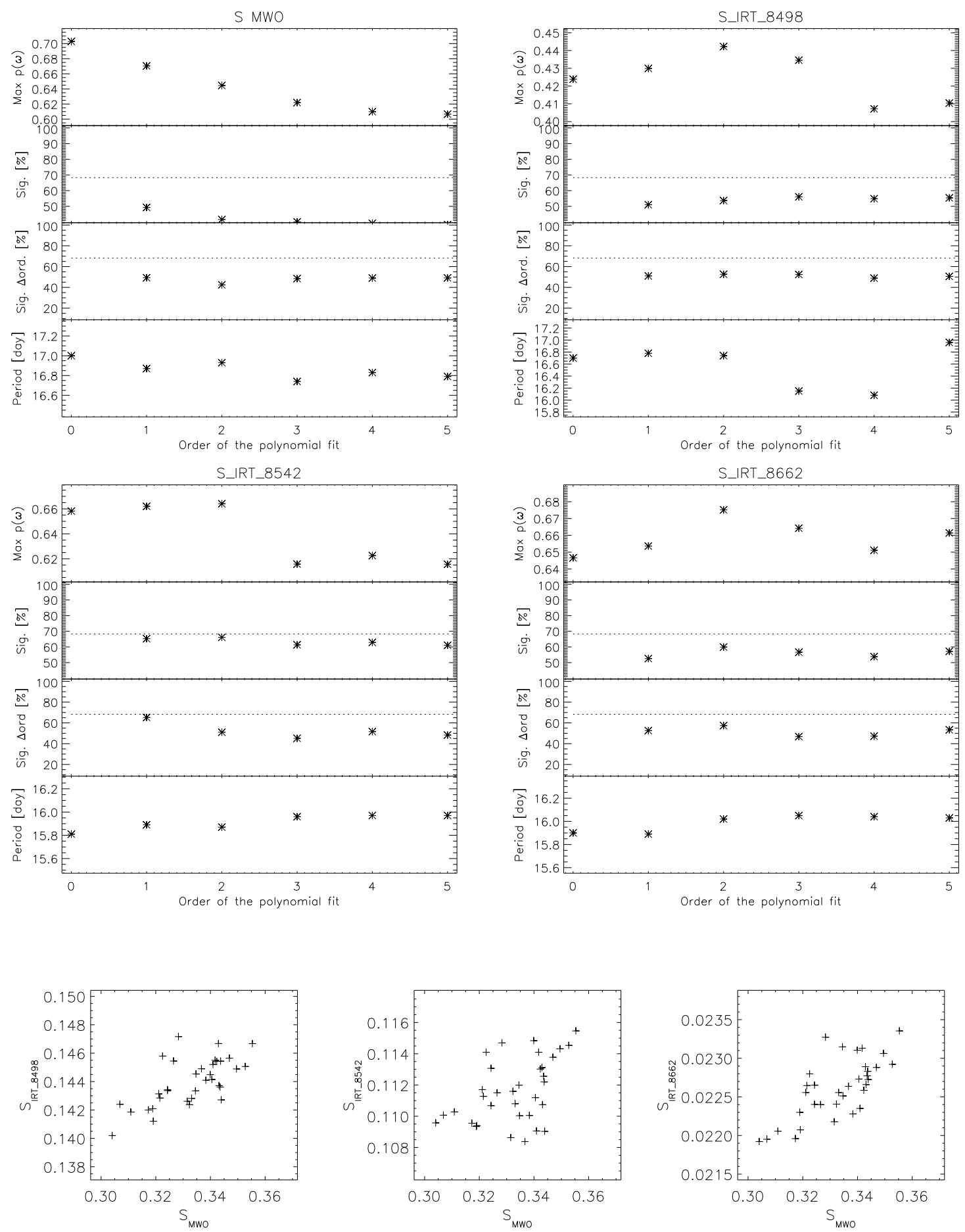

Fig. A.8. Plots for the time series analysis of HD 101501 and, for the detrending, the polynomial orders of $0,0,0$ and 0 are used for $S_{\mathrm{Mwo}}$, $S_{\text {IRT_8498 }}, S_{\text {IRT_8542 }}$ and $S_{\text {IRT_8662 }}$. A description of these figures can be found at the beginning of this Appendix. 
M. Mittag et al.: Comparison measurement of rotation periods with Ca II H\&K and Ca II IRT
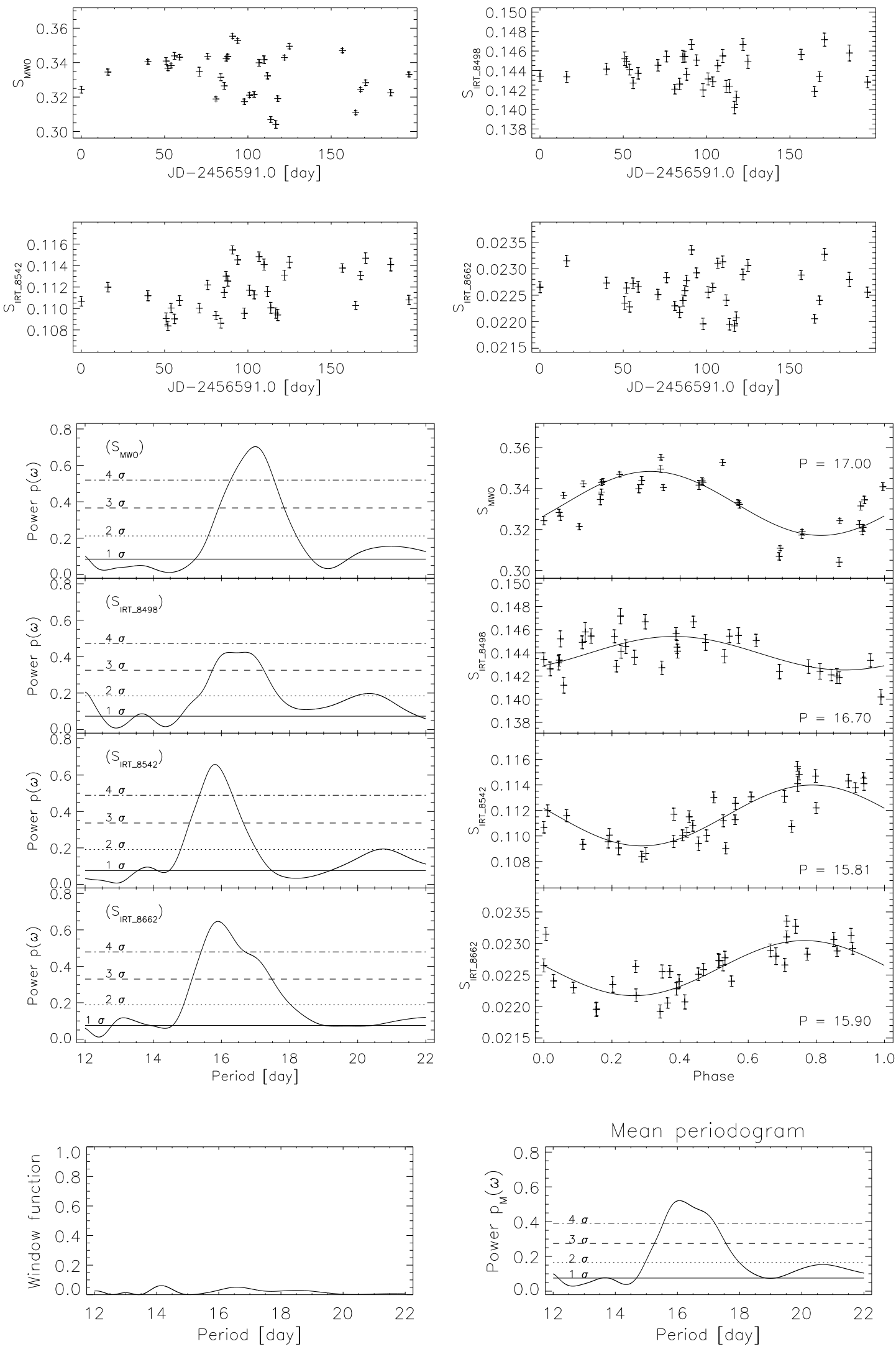

Fig. A.8. continued. 

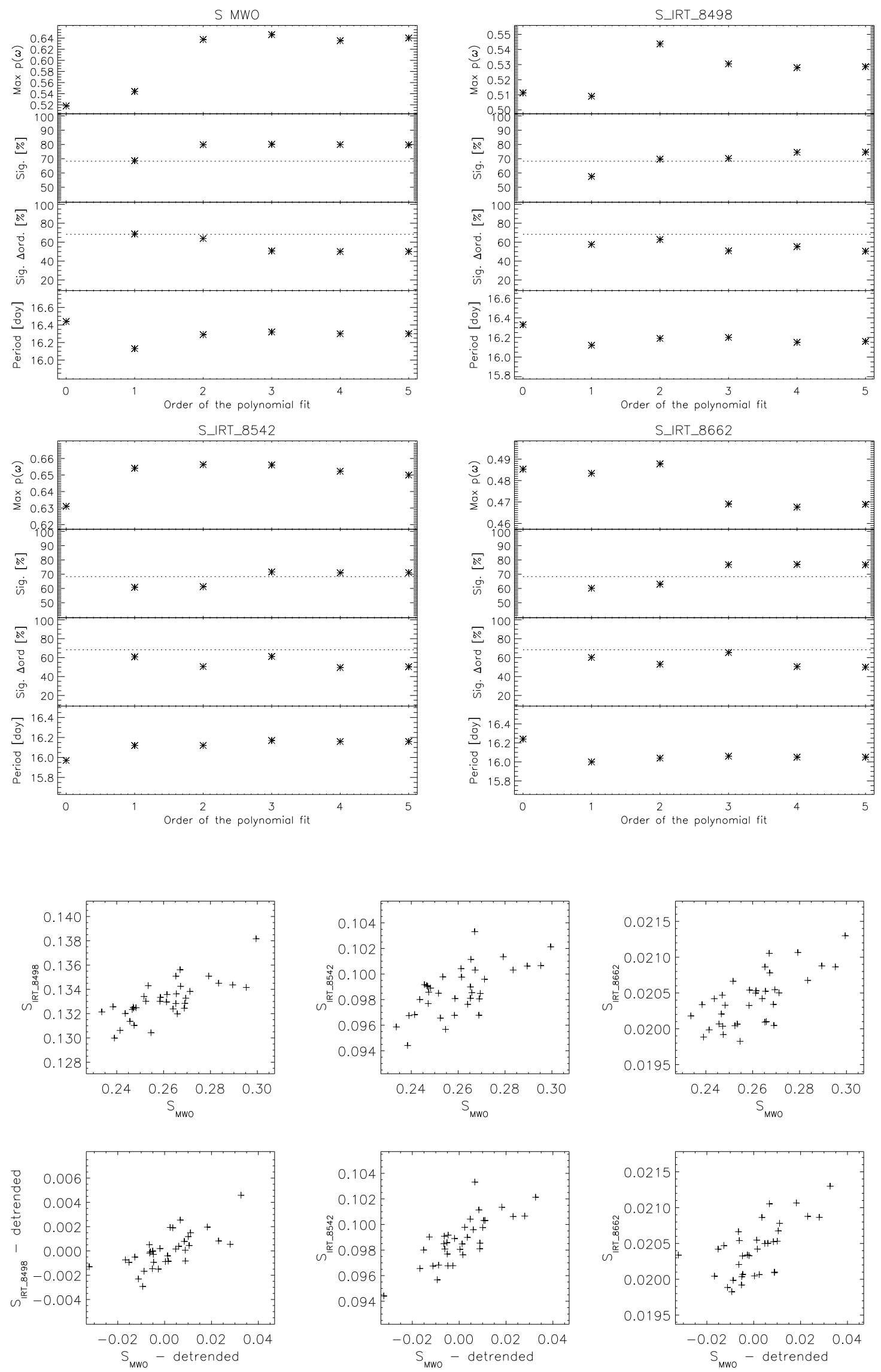

Fig. A.9. Plots for the time series analysis of HD 111395 and, for the detrending, the polynomial orders of 2, 2, 0 and 0 are used for $S_{\text {Mwo, }}$ $S_{\text {IRT_8498 }}, S_{\text {IRT_8542 }}$ and $S_{\text {IRT_8662 }}$. A description of these figures can be found at the beginning of this Appendix. 
M. Mittag et al.: Comparison measurement of rotation periods with Ca II H\&K and Ca II IRT
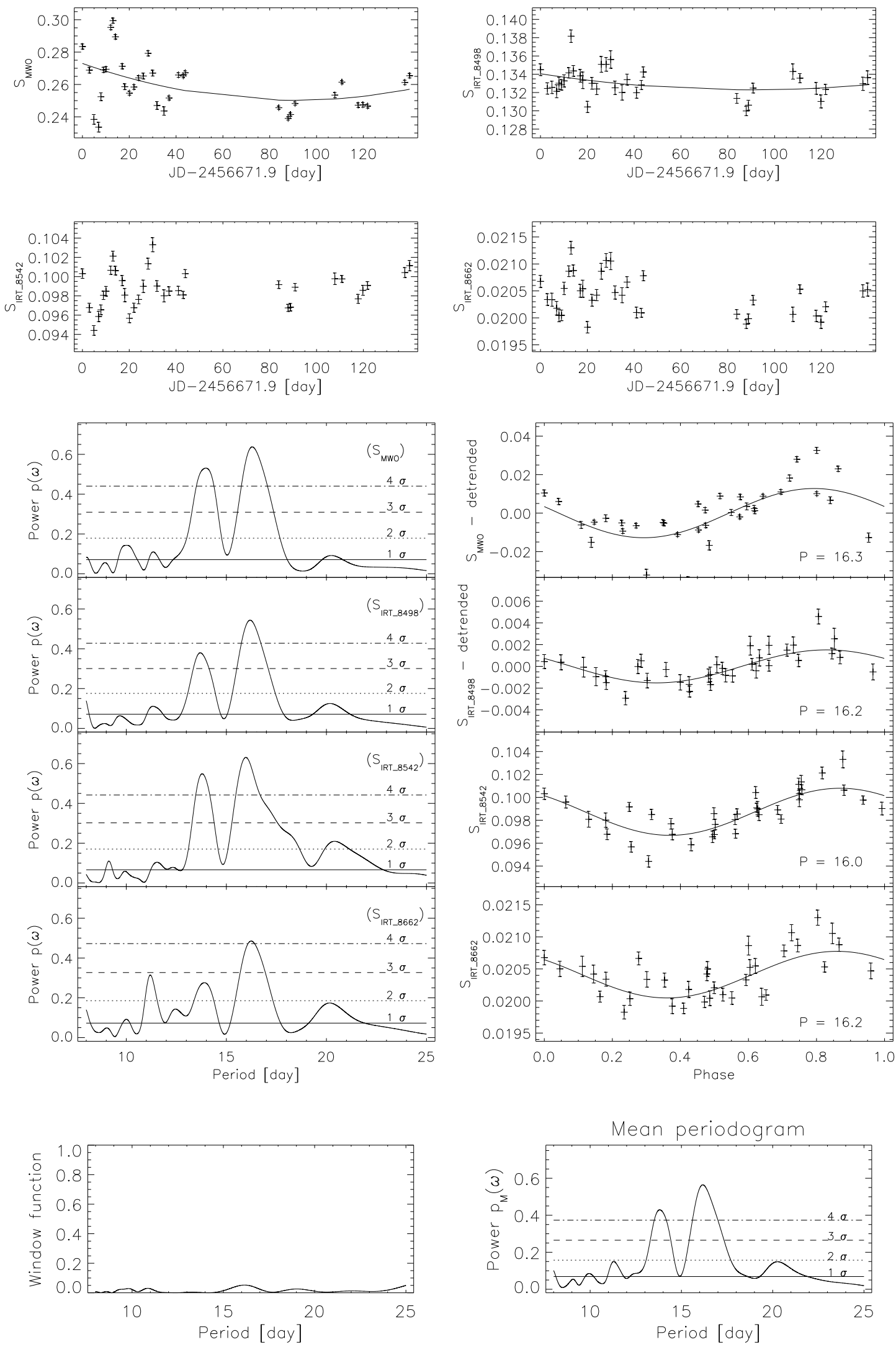

Fig. A.9. continued. 

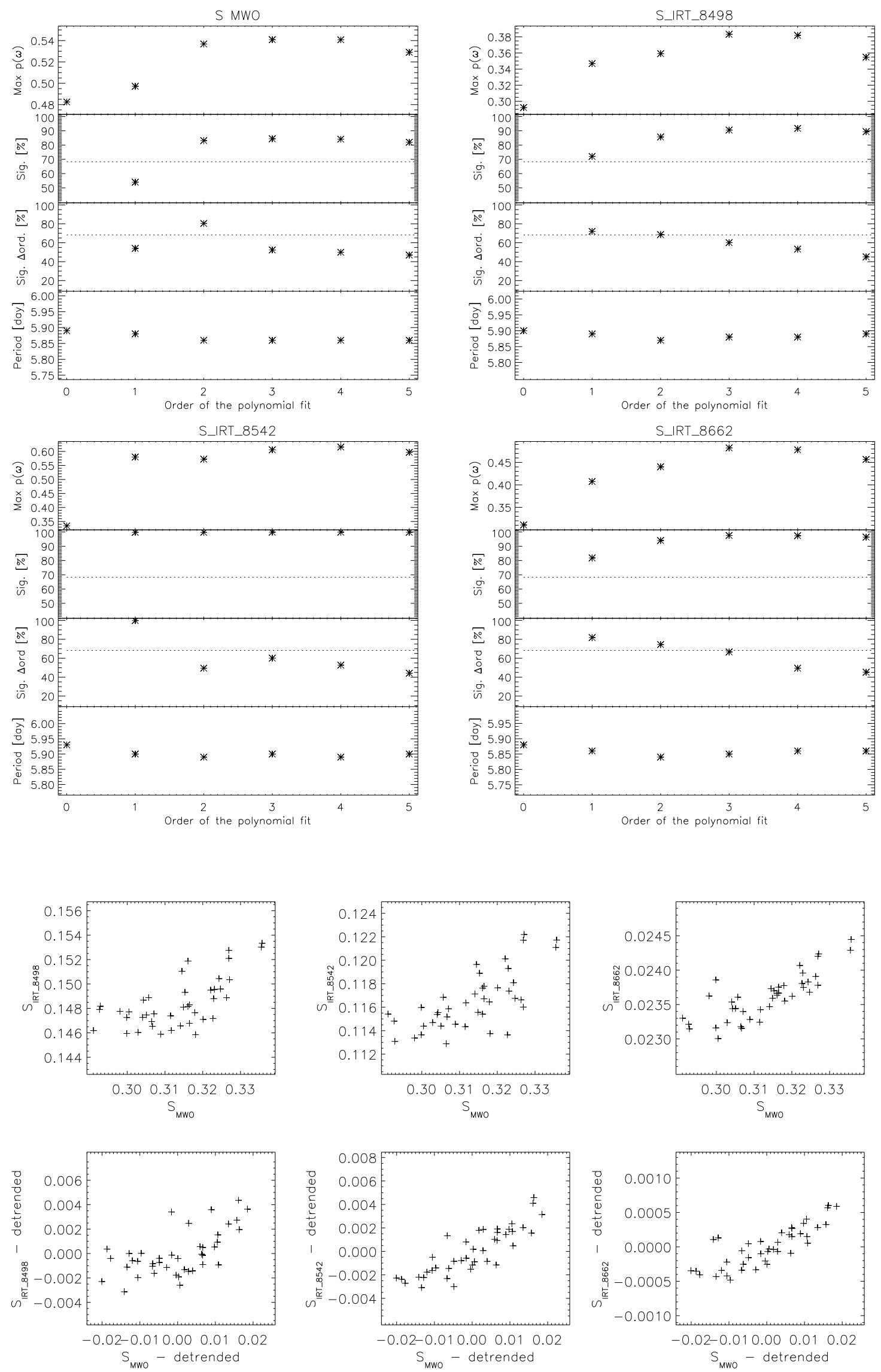

Fig. A.10. Plots for the time series analysis of HD 115043 and, for the detrending, the polynomial orders of 2, 2, 1 and 2 are used for $S_{\text {Mwo, }}$ $S_{\text {IRT_8498 }}, S_{\text {IRT_8542 }}$ and $S_{\text {IRT_8662 }}$. A description of these figures can be found at the beginning of this Appendix. 
M. Mittag et al.: Comparison measurement of rotation periods with Ca II H\&K and Ca II IRT
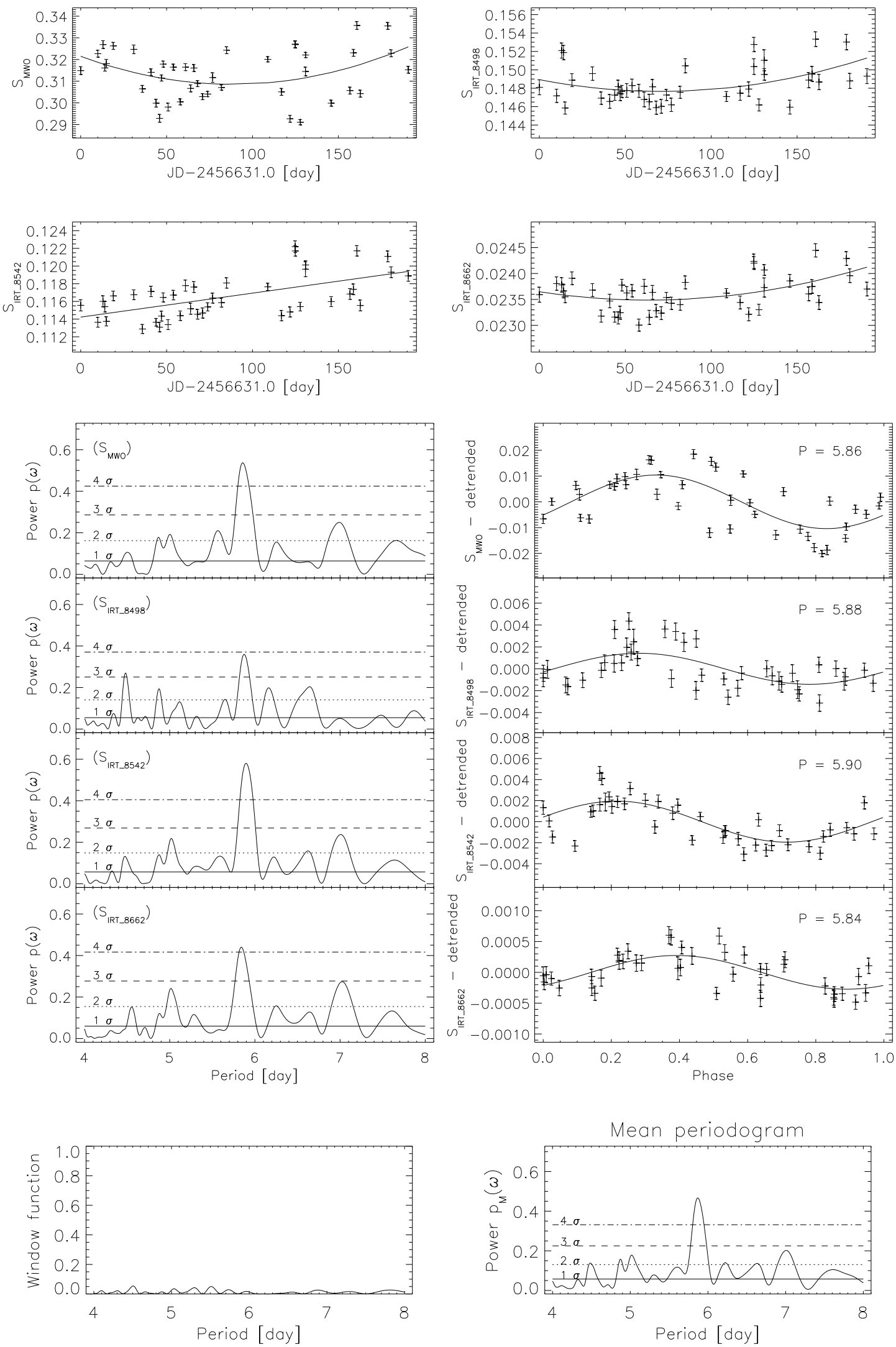

Fig. A.10. continued. 

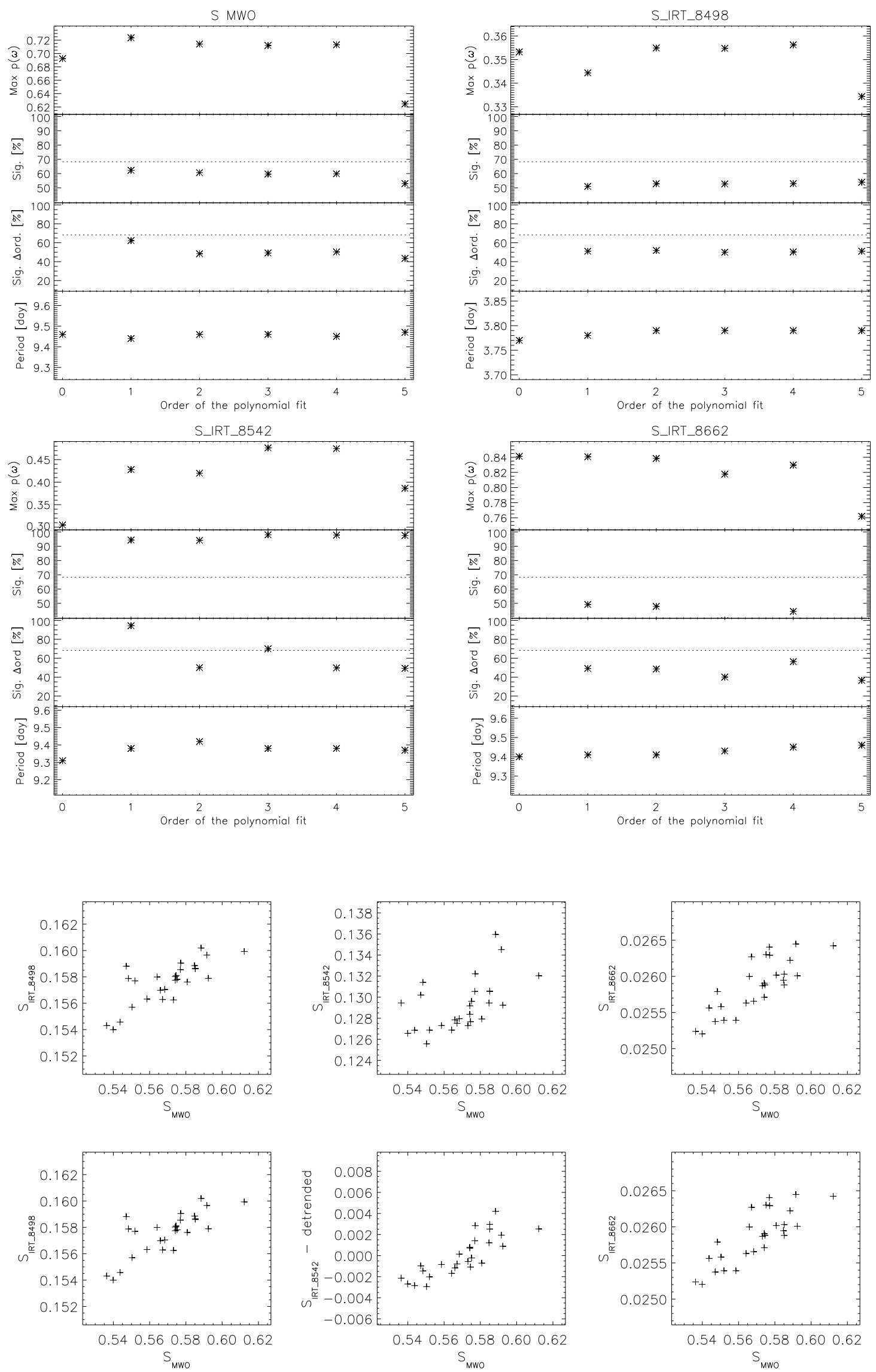

Fig. A.11. Plots for the time series analysis of HD 118972 and, for the detrending, the polynomial orders of $0,0,1$ and 0 are used for $S_{\text {Mwo, }}$ $S_{\text {IRT_8498 }}, S_{\text {IRT_8542 }}$ and $S_{\text {IRT_8662 }}$. A description of these figures can be found at the beginning of this Appendix. 
M. Mittag et al.: Comparison measurement of rotation periods with Ca II H\&K and Ca II IRT
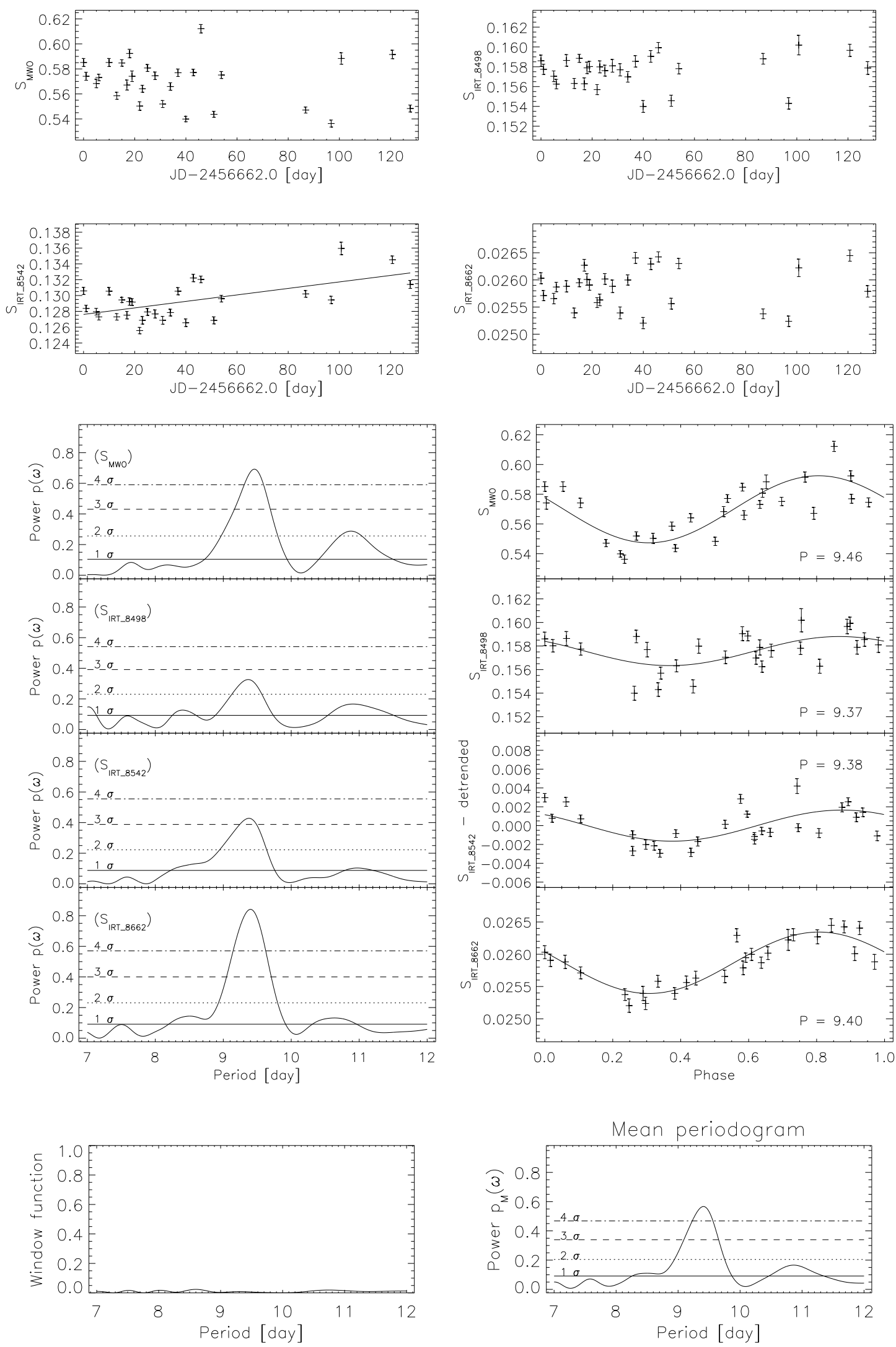

Fig. A.11. continued. 

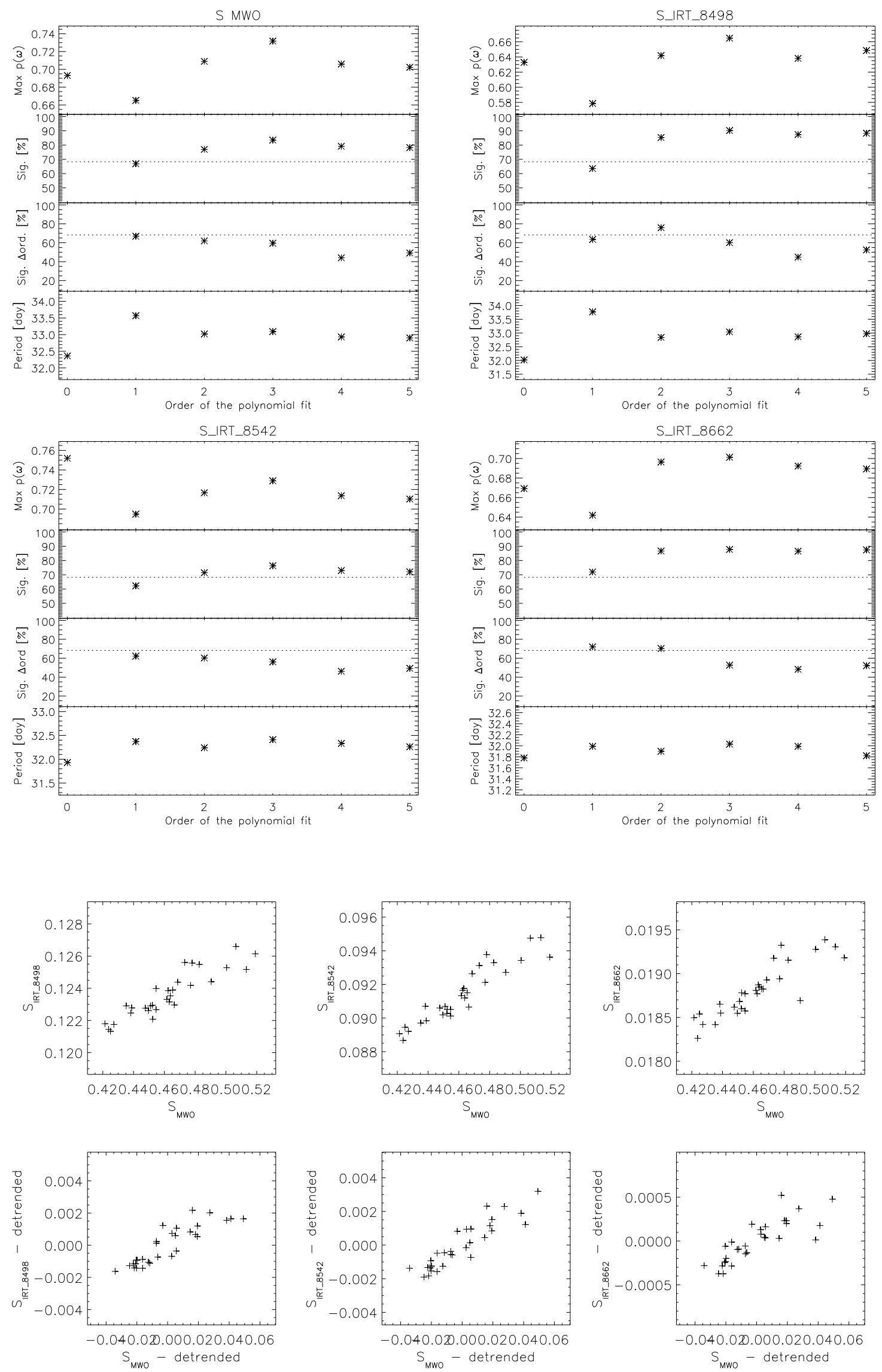

Fig. A.12. Plots for the time series analysis of HD 131977 and, for the detrending, the polynomial orders of 2, 2, 2 and 2 are used for $S_{\mathrm{Mwo}}$, $S_{\text {IRT_8498 }}, S_{\text {IRT_8542 }}$ and $S_{\text {IRT_8662 }}$. A description of these figures can be found at the beginning of this Appendix. 
M. Mittag et al.: Comparison measurement of rotation periods with Ca II H\&K and Ca II IRT
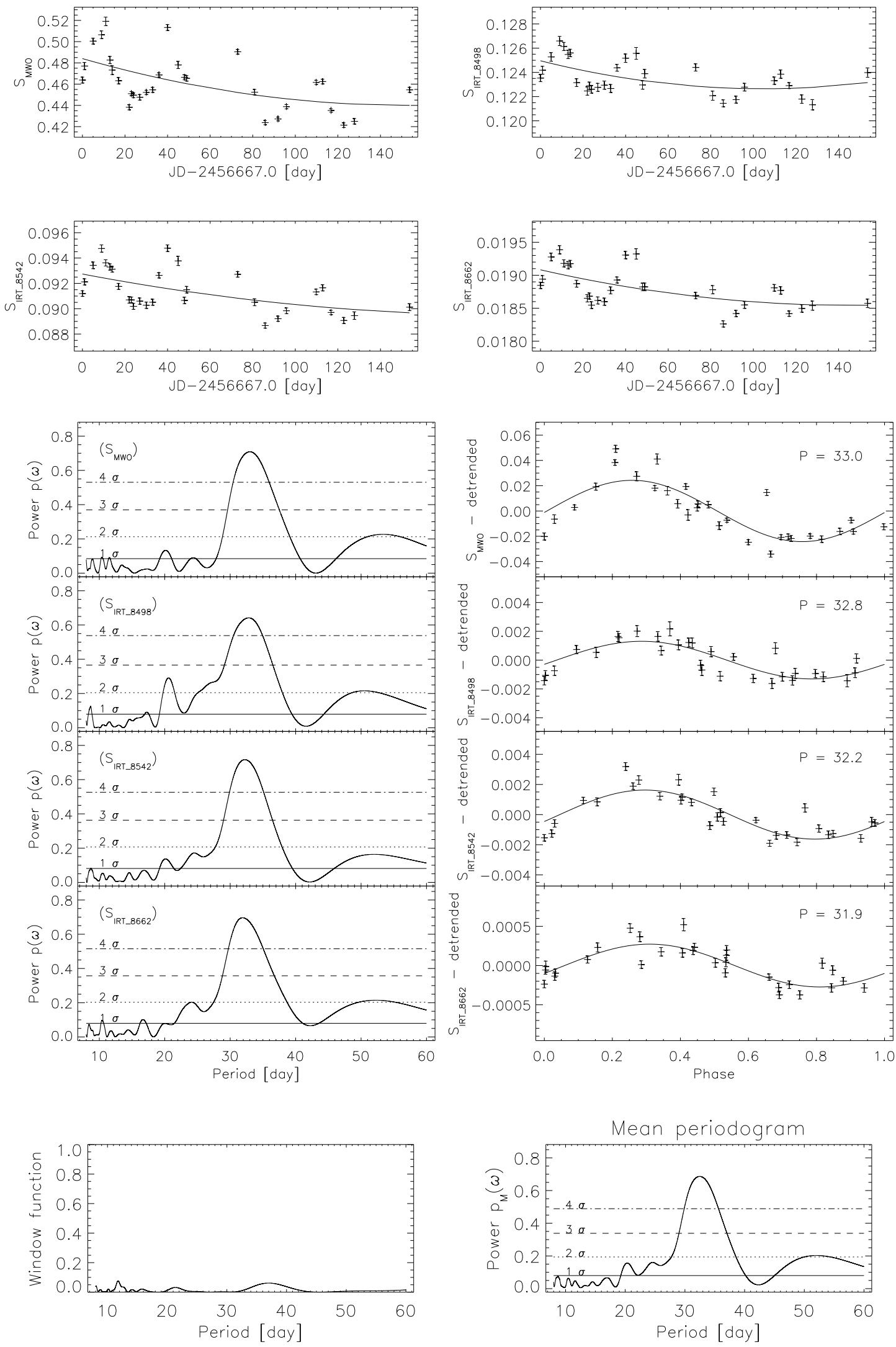

Fig. A.12. continued. 


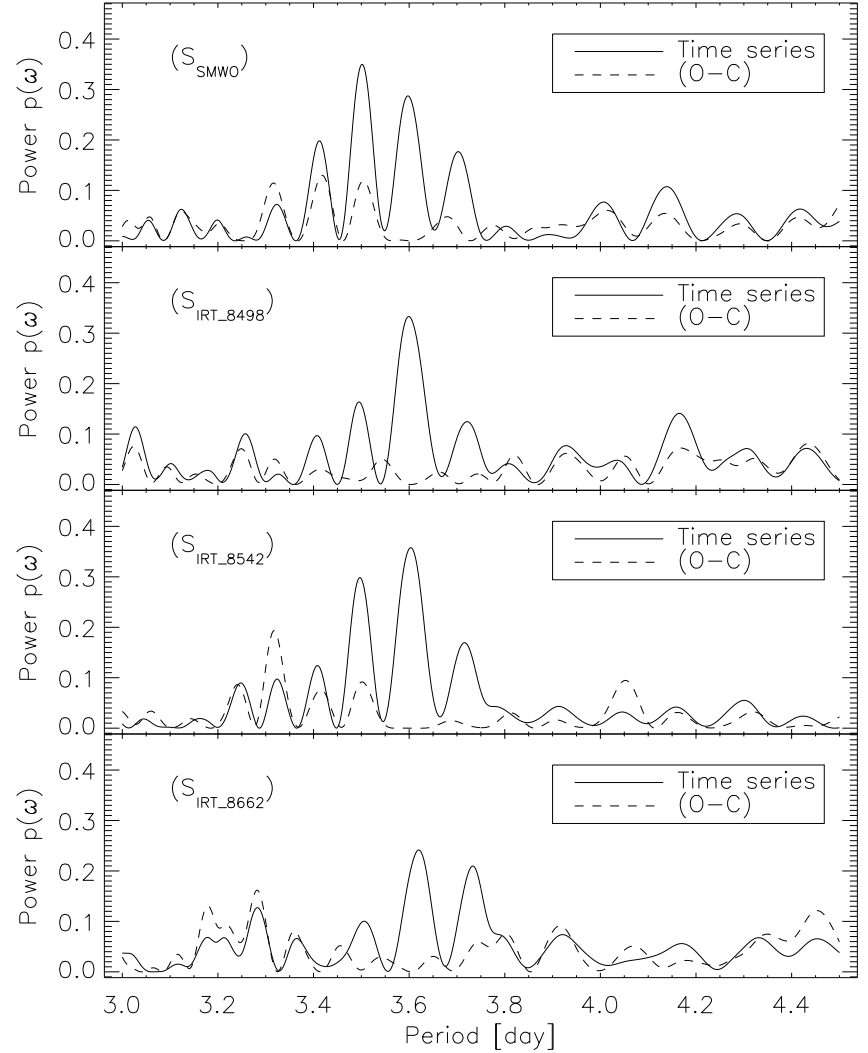

Fig. A.13. Periodograms for HD 35296 of the four time series without the removal of the most probable period (3.604 days) is depicted with the solid line and the data after the removal $(\mathrm{O}-\mathrm{C})$ with a dashed line.

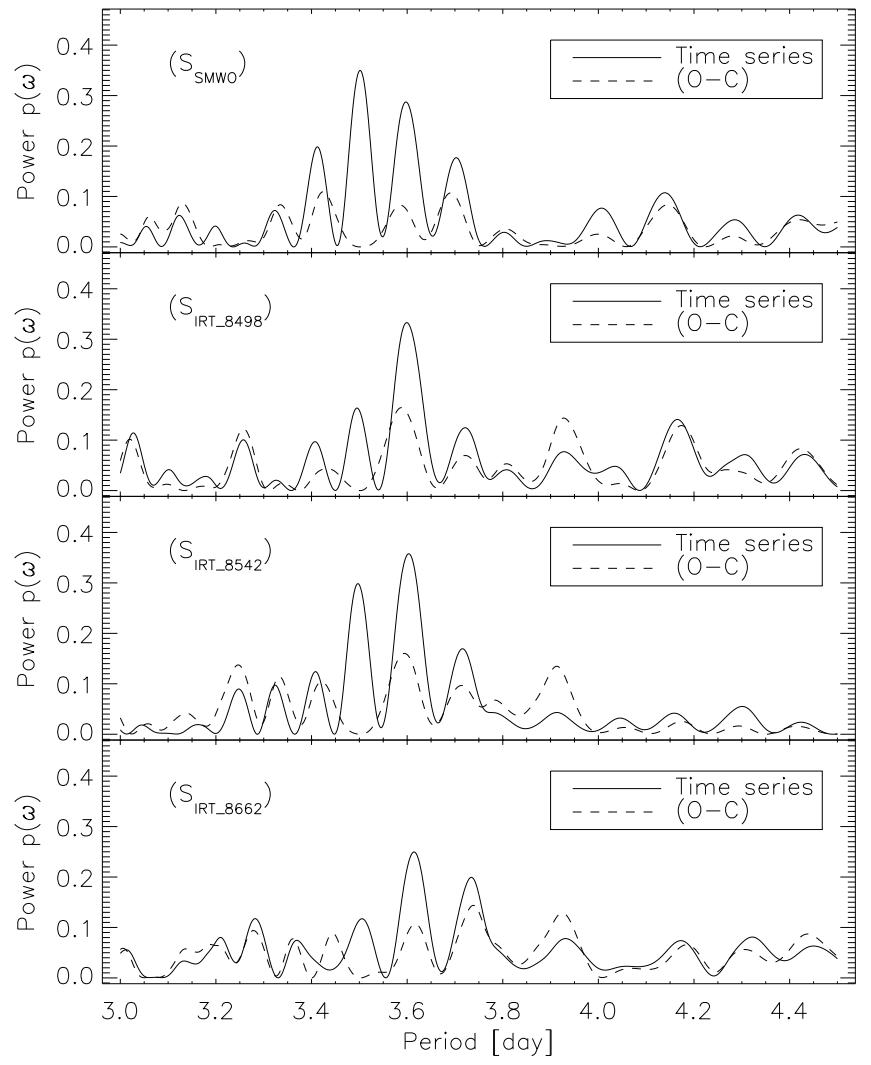

Fig. A.14. Periodograms for HD 35296 of the four time series without the removal of the 3.502 day period is depicted with the solid line and the data after the removal $(\mathrm{O}-\mathrm{C})$ with a dashed line.

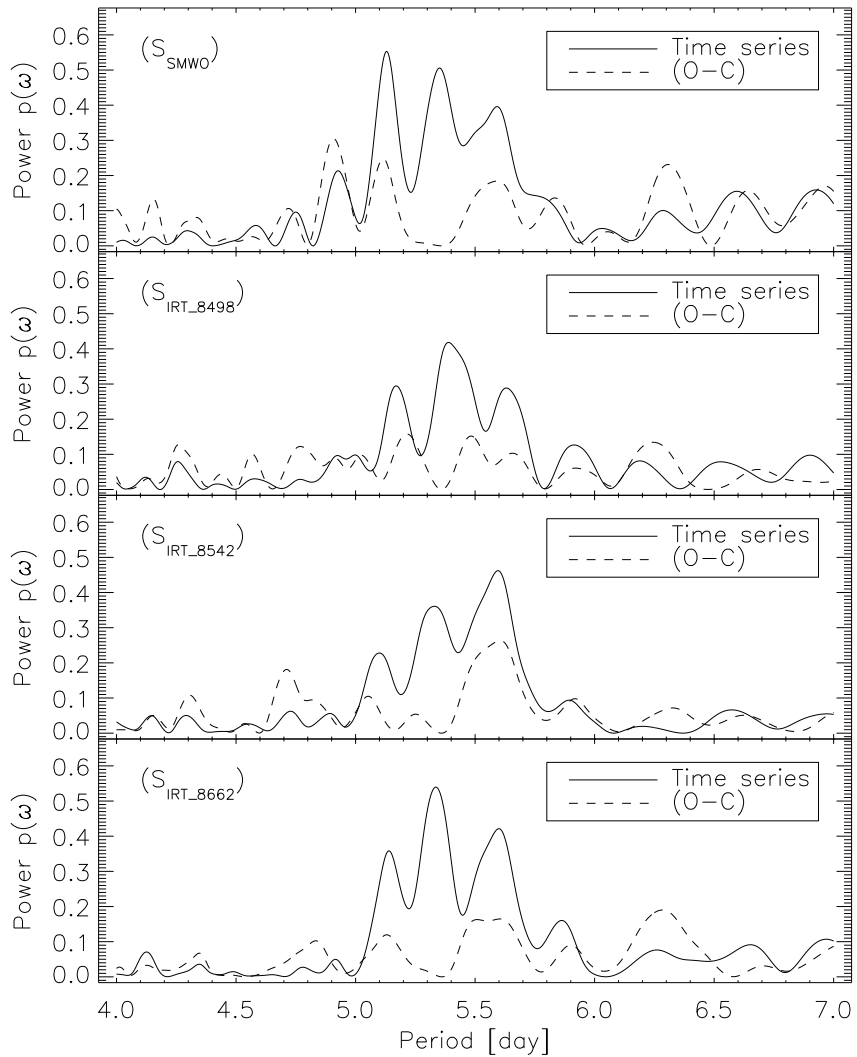

Fig. A.15. Periodograms for HD 39587 of the four time series without the removal of the most probable period (5.36 day) is depicted with the solid line and the data after the removal $(\mathrm{O}-\mathrm{C})$ with a dashed line.

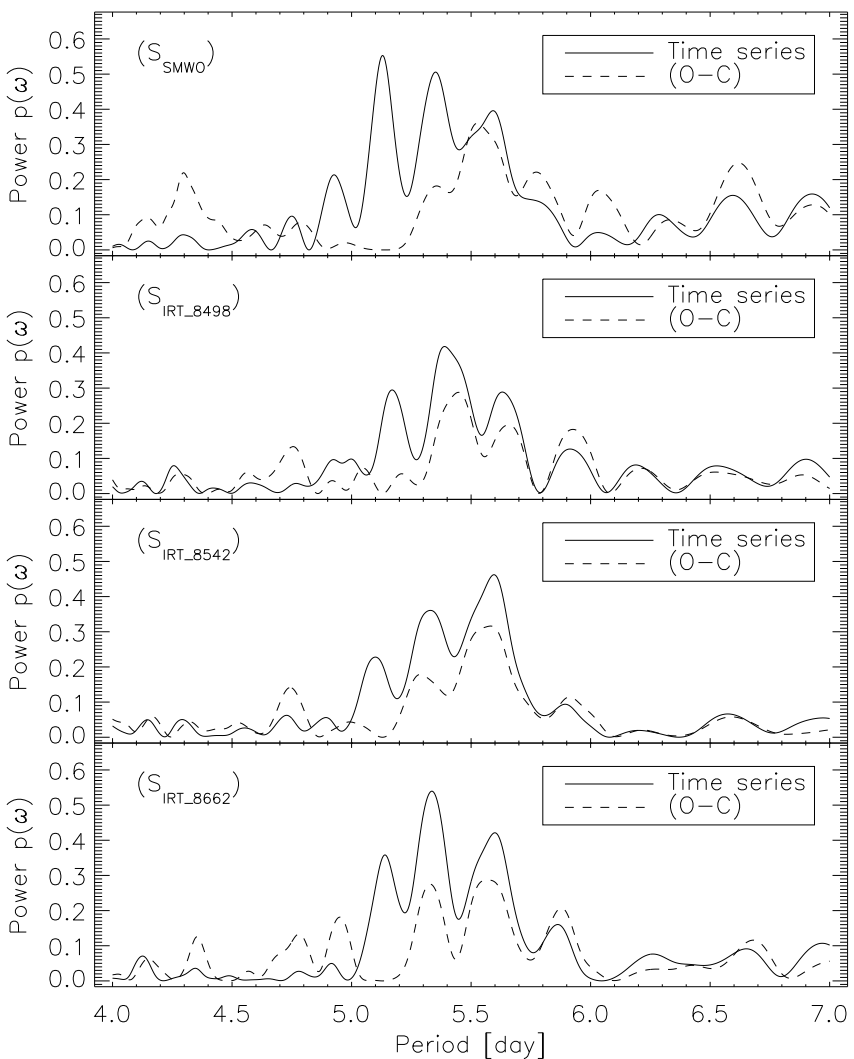

Fig. A.16. Periodograms for HD 39587 of the four time series without the removal of the 5.13 day period is depicted with the solid line and the data after removal $(\mathrm{O}-\mathrm{C})$ with a dashed line. 
M. Mittag et al.: Comparison measurement of rotation periods with Ca II H\&K and Ca II IRT

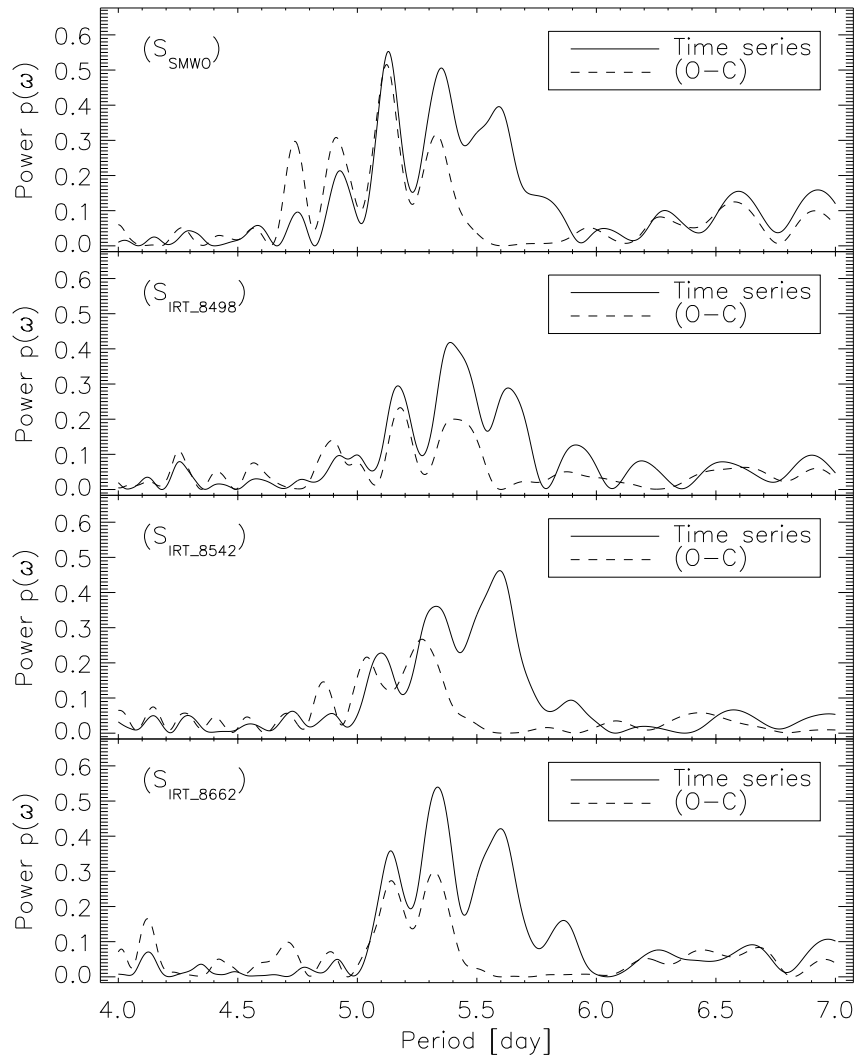

Fig. A.17. Periodograms for HD 39587 of the four time series without the removal of the 5.6 day period is depicted with the solid line and the data after the removal $(\mathrm{O}-\mathrm{C})$ with a dashed line.

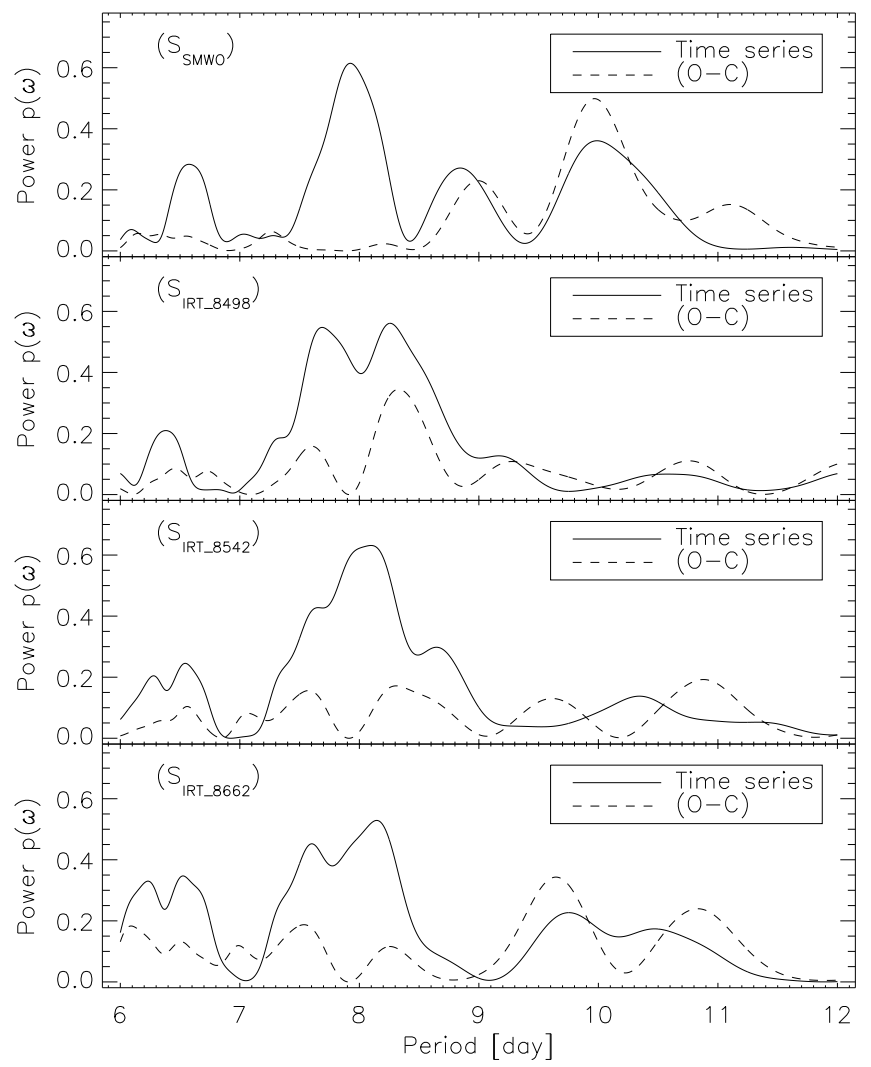

Fig. A.18. Periodograms for HD 97334 of the four time series without the removal of the 7.92 day period is depicted with the solid line and the data after removal $(\mathrm{O}-\mathrm{C})$ with a dashed line.

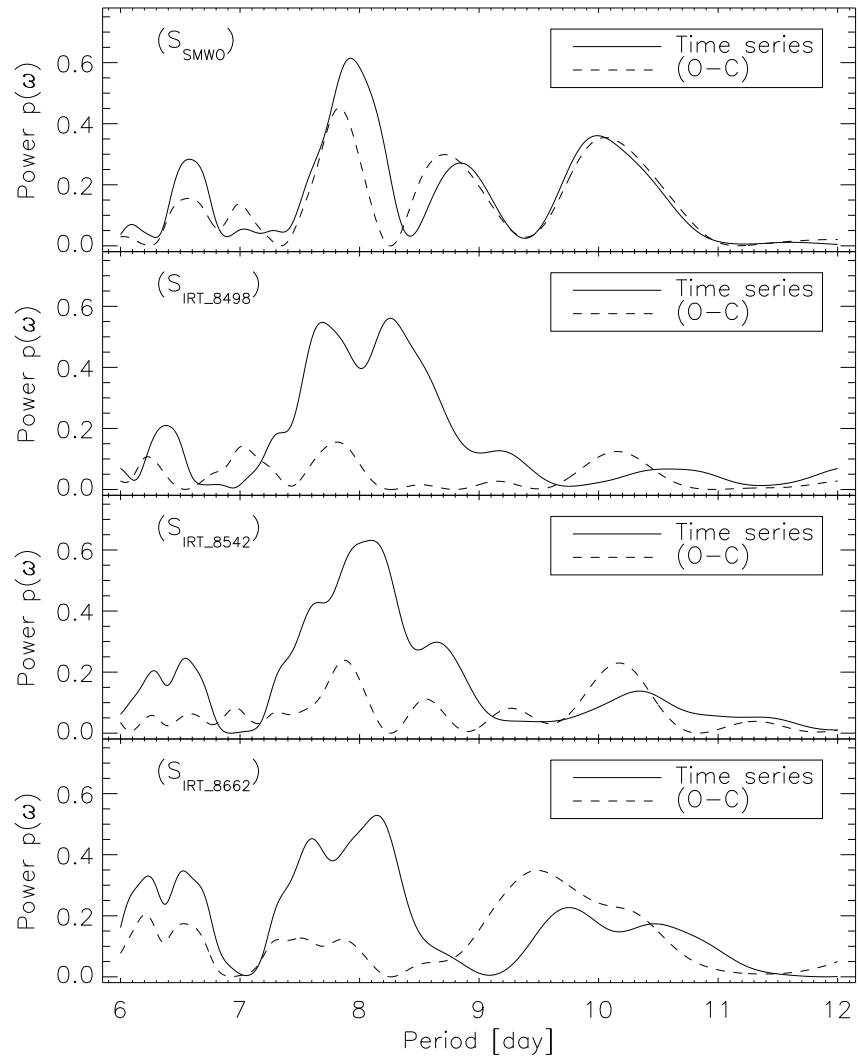

Fig. A.19. Periodograms for HD 97334 of the four time series without the removal of the 8.26 day period is depicted with the solid line and the data after removal $(\mathrm{O}-\mathrm{C})$ with a dashed line.

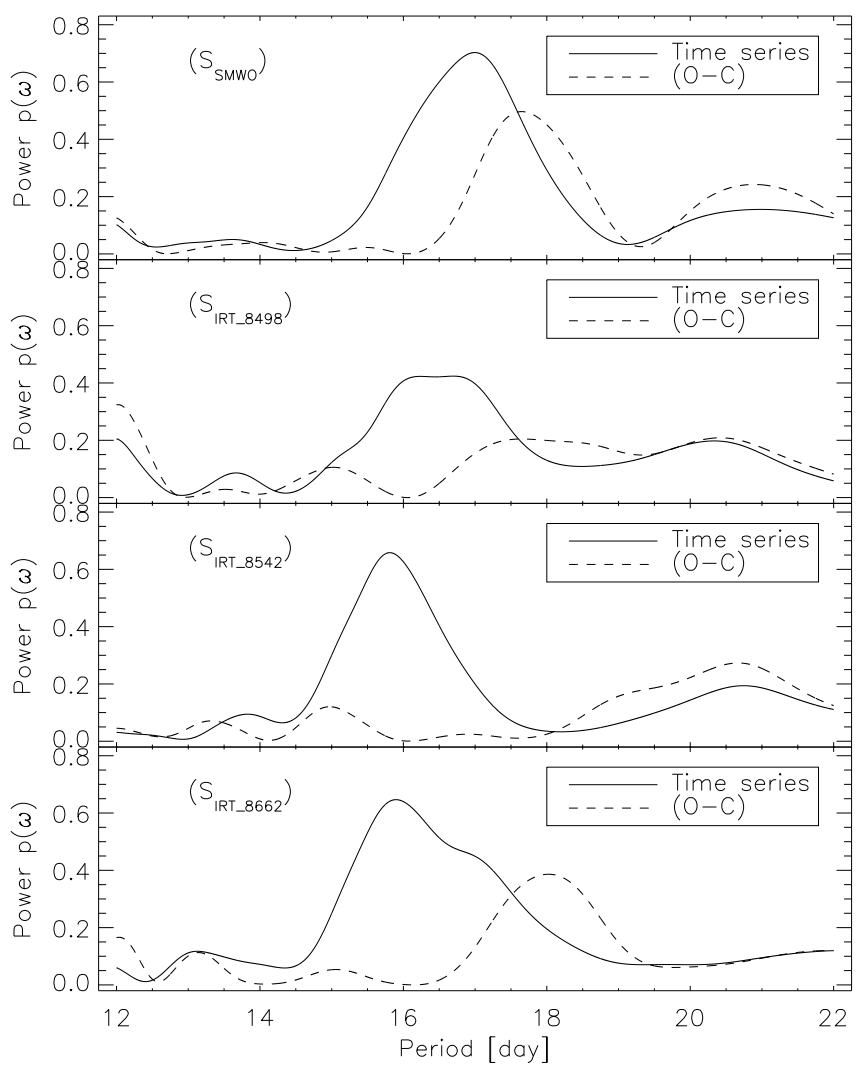

Fig. A.20. Periodograms for HD 101501 of the four time series without the removal of the most probable period (16.06 day) is depicted with the solid line and the data after the removal $(\mathrm{O}-\mathrm{C})$ with a dashed line. 